\title{
THREEFOLD EXTREMAL CURVE GERMS WITH ONE NON-GORENSTEIN POINT
}

\author{
SHIGEFUMI MORI AND YURI PROKHOROV
}

\begin{abstract}
An extremal curve germ is the analytic germ of a threefold with terminal singularities along a reduced complete curve admitting a contraction whose fibers have dimension at most one. The aim of the present paper is to review the results concerning those contractions whose central fiber is irreducible and contains only one non-Gorenstein point.
\end{abstract}

\section{Contents}

1. Introduction

2. Preliminaries

3. Basic techniques

4. Topological observations

5. Local description 15

6. Deformations 18

7. General member of $\left|-K_{X}\right|$

8. Index two germs 24

9. Locally imprimitive germs 31

10. Cases (IC) and (IIB) 36

11. Case (IA) 40

12. Case (IIA) 46

Appendix A. A remark on divisorial contractions 50

References 52

\section{INTRODUCTION}

One of the most important problems in the three-dimensional birational geometry is to describe explicitly all the steps of the Minimal

This work was supported by the Research Institute for Mathematical Sciences, a Joint Usage/Research Center located in Kyoto University. The first author was supported by the JSPS Grant Numbers JP25287005 and JP17H06127. The second author was supported by the Program of the Presidium of the Russian Academy of Sciences No. 01 "Fundamental Mathematics and its Applications" under grant PRAS-18-01 and the Russian Academic Excellence Project "5-100". 
Model Program (MMP). These steps consist of certain maps, called divisorial, flipping, and fiber-type contractions (Mori contractions). The structure of these maps is still unknown in its complete generality, though much progress has been made in this direction. We refer to [CKM88 for an introduction to the subject. The aim of the present paper is to review the results concerning those contractions whose fibers have dimensio $\mathrm{n}$ at most one. The project was started in the initial paper [Mor88] where the minimal model problem was solved in the three-dimensional case. To study Mori contractions in this situation one needs to work in the analytic category and analytic counterparts of the corresponding notions are needed. The central objects of this paper are so-called extremal curve germs.

An extremal curve germ is the analytic germ of a threefold with terminal singularities along a reduced complete curve admitting a contraction whose fibers have dimension at most one. The present paper is a survey of known results on the classification of objects of this type. Basically we concentrate on the case of irreducible central fiber with only one non-Gorenstein point. In this case the results are complete, however they are scattered in the literature. This is the main reason to write this survey.

The classification of extremal curve germs is done in terms of a general element $H$ of the linear system $\left|\mathscr{O}_{X}\right|$ of trivial Cartier divisors containing $C$. In many cases this element $H$ is a normal surface and then the threefold can be viewed as a one-parameter deformation of $H$.

A birational extremal curve germ $f:(X, C) \rightarrow(Z, o)$ is said to be semistable, if for a general member $D \in\left|-K_{Z}\right|$, the germ $D_{Z}:=\operatorname{Spec}_{Z} f_{*} \mathscr{O}_{D}$ is Du Val of type A [KM92]. The semistable case is subdivided into two cases $(\mathrm{k} 1 \mathrm{~A})$ and $(\mathrm{k} 2 \mathrm{~A})$ according to the number of non-Gorenstein points of $X$ on $C$. Other cases are called exceptional. It turns out that treating semistable and exceptional germs uses different approaches. For example, in the exceptional flipping case, KM92 provides relatively simple computations of flipped variety. For semistable germs these computations become more explicit; in the $(\mathrm{k} 2 \mathrm{~A})$ case, from the general member $H \in\left|\mathscr{O}_{X}\right|$ one can decide whether $(X, C)$ is flipping or divisorial [Mor02, Corollary 4.1] and furthermore describe the flipped variety [Mor02, Theorem 4.7] and $Z$ [Mor02, Theorem 4.5], respectively; the (k1A) case is similarly treated by [HTU17] under additional assumption " $b_{2}\left(X_{s}\right)=1$ " (see 11.4.6). According to local classification (see Propositions 5.4 and 5.5), a semistable extremal curve germ of type (k1A) can be of type (IA ${ }^{\vee}$ ) or (IA). They are treated in Sect. 8, 9, and 11.

Here are summary of some of the results. 
1.1. Theorem. Let $f:(X, C) \rightarrow(Z, o)$ be a flipping extremal curve germ with irreducible central fiber $C$ and let $H_{Z} \in\left|\mathscr{O}_{Z}\right|$ be a general hyperplane section containing $C$. Then $H_{Z}$ and $f^{-1}\left(H_{Z}\right)$ are normal and have rational singularities. The singularity $\left(H_{Z}, o\right)$ is log terminal except for the cases described in 11.5 .5 and 12.3.3. Moreover, $\left(H_{Z}, o\right)$ is a cyclic quotient singularity if and only if $(X, C)$ is semistable.

The case where $(X, C)$ is semistable follows from Lemma 9.3.1. If $(X, C)$ has only one non-Gorenstein point (i.e. of type $(\mathrm{k} 1 \mathrm{~A}))$, we can use explicit classification 8.2.1, 9.1.6, 10.4, 11.3, 11.5.2, 12.3, For the remaining (kAD) case we refer to [KM92, §9].

1.2. Theorem. Let $f:(X, C) \rightarrow(Z, o)$ be a divisorial extremal curve germ with irreducible central fiber $C$ and let $H_{Z} \in\left|\mathscr{O}_{Z}\right|$ be a general hyperplane section containing o. Then $\left(H_{Z}, o\right)$ is either a Du Val point, a rational log canonical point of type $\tilde{\mathrm{D}}$ (in the case 9.1.5), or a cyclic quotient singularity of class T. Moreover, the last two possibilities occurs only if $(X, C)$ has a locally imprimitive point or $(X, C)$ is semistable and has two non-Gorenstein points whose indices are not coprime.

Moreover, by Theorem 6.4 the singularity $(Z, o)$ is terminal. If $(X, C)$ has no covers étale in codimension one, then $(Z, o)$ is of index one and $H_{Z}$ is Du Val. In the semistable case the assertion as above follows from Lemma 9.3.1. It remains to consider locally imprimitive cases $\left(\mathrm{IA}^{\vee}\right)$ and $\left(\mathrm{II}^{\vee}\right)$ (see 9.1.5 and 9.1.6). Note that for a divisorial curve germ the surface $H:=f^{-1}\left(H_{Z}\right)$ can be non-normal (see e.g. Example 12.6).

For the $\mathbb{Q}$-conic bundle $f:(X, C) \rightarrow(Z, o)$ we can show that the base is Du Val of type A (Corollary 7.7.1). The proof uses the existence of a Du Val member $D \in\left|-K_{X}\right|$, see [MP08a], [MP09], and Theorem 7.3 ,

The paper was written during the second author's stay at RIMS, Kyoto University. The author is very grateful to the institute for the invitation, hospitality and good working environment.

\section{Preliminaries}

2.1. Threefold terminal singularities. Recall that a threedimensional terminal singularity of index $m$ is a quotient of an isolated hypersurface singularity by a cyclic group $\boldsymbol{\mu}_{m}$ of order $m$. More precisely, let $(X, P)$ be an analytic germ of a three-dimensional terminal singularity of index $m$. Then there exists a terminal singularity $\left(X^{\sharp}, P^{\sharp}\right)$ of index 1 and a cyclic $\boldsymbol{\mu}_{m}$-cover

$$
\left(X^{\sharp}, P^{\sharp}\right) \longrightarrow(X, P)
$$

which is étale outside $P$ [Rei83]. Moreover, the singularity $\left(X^{\sharp}, P^{\sharp}\right)$ can be embedded to $\left(\mathbb{C}^{4}, 0\right)$ so that its general hyperplane section is a 
surface Du Val singularity (thus $\left(X^{\sharp}, P^{\sharp}\right)$ is so-called cDV singularity). A detailed classification of all possibilities for equations of $X^{\sharp} \subset \mathbb{C}^{4}$ and the action of $\boldsymbol{\mu}_{m}$ was obtained in [Mor85] (see also [Rei87], [KSB88]).

Assume that $m>1$. Then the $\boldsymbol{\mu}_{m}$-action on $\left(X^{\sharp}, P^{\sharp}\right)$ will be analyzed. We fix a character $\chi$ generating $\operatorname{Hom}\left(\boldsymbol{\mu}_{m}, \mathbb{C}^{*}\right)=\mathbb{Z} / m \mathbb{Z}$. For a $\boldsymbol{\mu}_{m}$-semiinvariant $z$, we write

$$
\operatorname{wt}(z) \equiv a \bmod m
$$

if $g(z)=\chi(g)^{a} z$ for all $g \in \boldsymbol{\mu}_{m}$.

2.1.1. Theorem ([Mor85]). In the above notation the singularity $\left(X^{\sharp}, P^{\sharp}\right)$ is $\boldsymbol{\mu}_{m}$-isomorphic to a hypersurface $\phi=0$ in $\left(\mathbb{C}_{x_{1}, \ldots, x_{4}}^{4}, 0\right)$ such that for some $a, b \in \mathbb{Z}$ prime to $m$ one of the following holds:

(i) $\operatorname{wt}(x, \phi) \equiv(a, b,-a, 0,0) \bmod m$;

(ii) $m=4$, and $\operatorname{wt}(x, \phi) \equiv(a, b,-a, 2,2) \bmod m$.

In the case (ii) we say that $(X, P)$ is a point of type $\mathrm{cAx} / 4$.

Thus the locus $\Upsilon \subset \mathbb{C}^{4}$ of the points at which $\boldsymbol{\mu}_{m}$-action is not free is a coordinate axis which is not contained in $X^{\sharp}$. The following number

$$
\operatorname{aw}(X, P):=\operatorname{mult}_{0}\left(\left.\phi\right|_{\Upsilon}\right)
$$

is well defined and called the axial multiplicity of $(X, P)$.

2.2. Recall that a contraction is a proper surjective morphism $f: X \rightarrow$ $Z$ of normal varieties such that $f_{*} \mathscr{O}_{X}=\mathscr{O}_{Z}$.

2.2.1. Definition. Let $(X, C)$ be the analytic germ of a threefold with terminal singularities along a reduced complete curve. We say that $(X, C)$ is an extremal curve germ if there is a contraction

$$
f:(X, C) \rightarrow(Z, o)
$$

such that $C=f^{-1}(o)_{\text {red }}$ and $-K_{X}$ is $f$-ample. Furthermore, $f$ is called flipping if its exceptional locus coincides with $C$ and divisorial if its exceptional locus is two-dimensional. If $f$ is not birational, then $Z$ is a surface and $(X, C)$ is said to be a $\mathbb{Q}$-conic bundle germ [MP08a].

In general, we do not assume that $X$ is $\mathbb{Q}$-factorial. This is because the $\mathbb{Q}$-factoriality is not a local condition in the analytic category (see [Kaw88, §1]).

For future references we need the following easy example.

2.3. Example. Consider the following action of $\boldsymbol{\mu}_{m}$ on $\mathbb{P}_{x}^{1} \times \mathbb{C}_{u, v}^{2}$ :

$$
(x ; u, v) \longmapsto\left(\varepsilon^{a} x ; \varepsilon u, \varepsilon^{-1} v\right),
$$

where $\varepsilon$ is a primitive $m$-th root of unity and $\operatorname{gcd}(m, a)=1$. Let $X:=\mathbb{P}^{1} \times \mathbb{C}^{2} / \boldsymbol{\mu}_{m}, Z:=\mathbb{C}^{2} / \boldsymbol{\mu}_{m}$ and let $f: X \rightarrow Z$ be the natural projection. Since $\boldsymbol{\mu}_{m}$ acts freely in codimension one, $-K_{X}$ is $f$-ample. The images of two fixed points on $\mathbb{P}^{1} \times \mathbb{C}^{2}$ are terminal cyclic quotient 
singularities of types $\frac{1}{m}( \pm a, 1,-1)$ on $X$. Hence, $f$ is a $\mathbb{Q}$-conic bundle. A $\mathbb{Q}$-conic bundle germ biholomorphic to $f$ as above is called toroidal.

The following key fact is an immediate consequence of the KawamataViehweg vanishing theorem.

2.4. Theorem. Let $f:(X, C) \rightarrow(Z, o)$ be an extremal curve germ. Then $R^{i} f_{*} \mathscr{O}_{X}=0$ for $i>0$.

2.4.1. Corollary (cf. Mor88, Remark 1.2.1, Cor. 1.3]). (i) If $\mathscr{J}$ is an ideal such that $\operatorname{Supp}\left(\mathscr{O}_{X} / \mathscr{J}\right) \subset C$, then $H^{1}\left(\mathscr{O}_{X} / \mathscr{J}\right)=0$.

(ii) $p_{\mathrm{a}}(C)=0$ and $C$ is a union of smooth rational curves.

(iii) $\operatorname{Pic} X \simeq H^{2}(C, \mathbb{Z}) \simeq \mathbb{Z}^{\rho}$, where $\rho$ is the number of irreducible components of $C$.

2.4.2. Remark. If $C$ is reducible, then for every proper curve $C^{\prime} \subsetneq C$, the germ $\left(X, C^{\prime}\right)$ is also an extremal curve germ.

2.5. Lemma. Let $f:(X, C) \rightarrow(Z, o)$ be an extremal curve germ.

(i) If $f$ is birational, then on $Z$ there exists an effective $\mathbb{Q}$-divisor $B$ such that the pair $(Z, B)$ has only canonical singularity at $o$. If moreover $f$ is flipping, then the singularity of $(Z, B)$ at $o$ is terminal.

(ii) If $f$ is a $\mathbb{Q}$-conic bundle, then $Z$ has a log terminal singularity at $o$.

Proof. Take $n \gg 0$ so that the divisor $n K_{X}$ is Cartier and the linear system $\left|-n K_{X}\right|$ is base point free. Let $H \in\left|-n K_{X}\right|$ be a general member. Then $H$ is a smooth surface meeting the components of $C$ transversally. For (i), put $D:=\frac{1}{n} H$ and $B:=f_{*} D$. Then the singularities of the pair $(X, D)$ are terminal. Since $f$ is crepant with respect to $K_{X}+D$ and does not contract components of $D$, we see that the singularities of $(Z, B)$ are canonical [KM98, Lemma 3.38]. To show (ii) we note that the restriction $f_{H}: H \rightarrow Z$ is a finite morphism. Thus $(Z, o)$ is a log terminal singularity [KM98, Prop. 5.20].

Note however that in (i) we do not assert that the point $(Z, o)$ is $\mathbb{Q}$-Gorenstein, even in the divisorial case, see Theorem 6.4. The result of (ii) is significantly improved in 4.6.5 and [MP08a, 1.2.7].

2.6. General member of $\left|\mathscr{O}_{X}\right|$. Let $f:(X, C) \rightarrow(Z, o)$ be an extremal curve germ. If $f$ is a $\mathbb{Q}$-conic bundle, then we assume that $(Z, o)$ is smooth. We denote by $\left|\mathscr{O}_{Z}\right|$ the linear system of Cartier divisors (hyperplane sections) passing through $o$ and $\left|\mathscr{O}_{X}\right|:=f^{*}\left|\mathscr{O}_{Z}\right|$. Let $H$ be a general member of $\left|\mathscr{O}_{X}\right|$ and let $H_{Z}=f(H)$. Let $H^{\mathrm{n}} \rightarrow H$ be the normalization (we put $H^{\mathrm{n}}=H$ if $H$ is normal). By [KM98, 5.25] and Lemma 2.5 both $H_{Z}$ and $H$ are Cohen-Macaulay. Hence by Bertini's 
theorem $H_{Z}$ is normal. Then the composition map $H^{\mathrm{n}} \rightarrow H_{Z}$ has connected fibers. Moreover, it is a rational curve fibration if $\operatorname{dim} Z=2$; a birational contraction to a point $\left(H_{Z}, o\right)$ if $f$ is birational. Thus in the $\mathbb{Q}$-conic bundle case $H^{\mathrm{n}}$ has only rational singularities. The same is true in the birational case if the singularity $\left(H_{Z}, o\right)$ is rational.

2.7. Notation on dual graphs. Let $S$ be a normal surface and let $C \subset S$ be a curve. Suppose that on the minimal resolution of $S$ the exceptional divisors and the proper transform of $C$ form a normal crossing divisor, say $R$. We use the usual notation of dual graphs $\Delta(S, C)$ of $R$ : each $\diamond$ corresponds to an irreducible component of $C$ and each $\circ$ corresponds to an exceptional divisor, and we may use $\bullet$ instead of $\diamond$ if we want to emphasize that it is a complete $(-1)$-curve. A number attached to a vertex denotes the minus self-intersection number. For short, we may omit 2 if the self-intersection equals -2 .

2.7.1. Proposition ([Cut88]). $\quad$ (i) Let $f: X \rightarrow Z$ be a $\mathbb{Q}$-conic bundle. If $X$ is Gorenstein (and terminal), then $Z$ is smooth and there is a vector bundle $\mathscr{E}$ of rank 3 on $Z$ and an embedding $X \hookrightarrow \mathbb{P}(\mathscr{E})$ such that every scheme fiber $X_{z}, z \in Z$ is a conic in $\mathbb{P}(\mathscr{E})_{z}$.

(ii) (see also [KM92, 4.7.2]) Let $f:(X, C) \rightarrow(Z, o)$ be a birational curve germ such that $X$ is Gorenstein. Then $f$ is divisorial, $(Z, o)$ is smooth, $C$ is irreducible, and $f$ is the blowup of a curve $B \subset Z$ having only planar singularities. Moreover, $X$ has exactly one singular point which is of type cA and for a general member $H \in\left|\mathscr{O}_{X}\right|$ the graph $\Delta(H, C)$ has the form

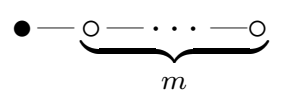

The following fact is a particular case of [KM92, Theorem 4.9].

2.8. Theorem. Let $f:(X, C) \rightarrow(Z, o)$ be a divisorial extremal curve germ. Let $E$ be its exceptional locus (with reduced structure) and let $B:=f(E)_{\mathrm{red}}$. Assume that $K_{Z}$ is $\mathbb{Q}$-Cartier (this automatically holds if $C$ is irreducible, see 6.4). Then the following holds.

(i) The set $E$ of $f$ is purely two-dimensional and is a $\mathbb{Q}$-Cartier divisor, and the singularity $(Z, o)$ is terminal.

(ii) The variety $X$ is the symbolic blowup of $B$, that is,

$$
X=\operatorname{Proj}_{Z} \bigoplus_{m=0}^{\infty} \mathcal{J}_{B}^{(m)},
$$

where $\mathcal{J}_{B}$ is the ideal sheaf of $B$, and $\mathcal{J}_{B}^{(m)}$ denotes its symbolic power. In particular, $X$ is uniquely determined by $B \subset Z$. 
It is possible to study divisorial curve germs algebraically, by scrupulous analysis of the curve $B$ and its embedding $B \subset X$ (see [Tzi03, [Tzi05a, Kaw96], [Tzi10], [PR16, § 6.1], [Duc16]). This method is completely different from our approach.

\section{BASIC TECHNIQUES}

3.1. Let $\mathcal{J}_{C} \subset \mathscr{O}_{X}$ be the ideal sheaf of $C$ and let $\mathcal{J}_{C}^{(n)}$ be its symbolic $n$th power, that is, the saturation of $\mathcal{J}_{C}^{n}$ in $\mathscr{O}_{X}$. Put

$$
\operatorname{gr}_{C}^{n} \mathscr{O}:=\mathfrak{J}_{C}^{(n)} / \mathcal{J}_{C}^{(n+1)} .
$$

Further, let $F^{n} \omega_{X}$ be the saturation of $\mathcal{J}_{C}^{n} \omega_{X}$ in $\omega_{X}$ and let

$$
\operatorname{gr}_{C}^{n} \omega:=F^{n} \omega_{X} / F^{n+1} \omega_{X} .
$$

Let $m$ be the index of $K_{X}$. We have natural homomorphisms

$$
\begin{aligned}
& \alpha_{1}: \bigwedge^{2} \operatorname{gr}_{C}^{1} \mathscr{O} \longrightarrow \mathscr{H}_{o m_{\mathscr{O}_{C}}}\left(\Omega_{C}^{1}, \operatorname{gr}_{C}^{0} \omega\right), \\
& \beta_{0}:\left(\operatorname{gr}_{C}^{0} \omega\right)^{\otimes m} \longrightarrow\left(\omega_{X}^{\otimes m}\right)^{\vee \vee} \otimes \mathscr{O}_{C} .
\end{aligned}
$$

Denote

$$
i_{P}(1):=\operatorname{len}_{P} \operatorname{Coker}\left(\alpha_{1}\right), \quad w_{P}(0):=\operatorname{len}_{P} \operatorname{Coker}\left(\beta_{0}\right) / m .
$$

To study extremal germs more carefully, Mori [Mor88 introduced also series of local invariants $i_{P}(n), w_{P}(n), w_{P}^{*}(n)$ similar to $i_{P}(1)$ and $w_{P}(0)$. We do not define them here.

Assume that $C \simeq \mathbb{P}^{1}$. Then we have by [Mor88, 2.3.1]

$$
\begin{aligned}
& -\operatorname{deg} \operatorname{gr}_{C}^{0} \omega=-K_{X} \cdot C+\sum_{P} w_{P}(0), \\
& 2+\operatorname{deg} \operatorname{gr}_{C}^{0} \omega-\operatorname{deg} \operatorname{gr}_{C}^{1} \mathscr{O}=\sum_{P} i_{P}(1) .
\end{aligned}
$$

Since $\operatorname{rkgr}_{C}^{1} \mathscr{O}=2$, taking 2.4 into account we obtain

$$
\operatorname{deg} \operatorname{gr}_{C}^{1} \mathscr{O} \geq-2
$$

$$
4 \geq-\operatorname{deg} \operatorname{gr}_{C}^{0} \boldsymbol{\omega}+\sum_{P} i_{P}(1)=-K_{X} \cdot C+\sum_{P} w_{P}(0)+\sum_{P} i_{P}(1)
$$

3.1.6. Remark. In the case where $f$ is birational, by the GrauertRiemenshneider vanishing, one has $\operatorname{gr}_{C}^{0} \omega=\mathscr{O}_{C}(-1)$ (see [Mor88, 2.3]). This is no longer true for $\mathbb{Q}$-conic bundles: in the toroidal example 2.3 easy computations show $\operatorname{deg} \operatorname{gr}_{C}^{0} \omega=-2$ (see (3.1.2)). Similarly, in the case 9.1 .2 we also have $\operatorname{deg} \operatorname{gr}_{C}^{0} \omega=-2$. We will show below that these two examples are the only exceptions (see Corollaries 4.7.8 and 5.5.1). 
3.2. Let $(X, P)$ be a germ of threefold terminal singularity. Throughout this paper $\left(X^{\sharp}, P^{\sharp}\right) \rightarrow(X, P)$ denotes the index-one cover. For any object $V$ on $X$ we denote by $V^{\sharp}$ the pull-back of $V$ on $X^{\sharp}$.

3.3. Lemma ([Mor88, 2.16]). In the above notation, assume that $C^{\sharp}$ is smooth. Denote

$$
\ell(P):=\operatorname{len}_{P} \mathrm{~J}_{C}^{\sharp(2)} / \mathrm{J}_{C}^{\sharp 2},
$$

where $\mathfrak{J}_{C}^{\sharp}$ is the ideal of $C^{\sharp}$ in $X^{\sharp}$. Then

$$
i_{P}(1)= \begin{cases}\ell(P) & \text { if } m=1, \\ \lfloor(\ell(P)+6) / 4\rfloor & \text { if }(X, P) \text { is of type } \mathrm{cAx} / 4, \\ \lfloor\ell(P) / m\rfloor+1 & \text { if }(X, P) \text { is not as above. }\end{cases}
$$

3.4. Lemma ([Mor88, 2.10, 2.15]). If $(X, P)$ is singular, then $i_{P}(1) \geq 1$. If $(X, P)$ is not Gorenstein, then $w_{P}(0)>0$.

Then from (3.1.5) we obtain

3.4.1. Corollary. An extremal curve germ $\left(X, C \simeq \mathbb{P}^{1}\right)$ has at most three singular points.

3.5. Let $(X, C)$ be an extremal curve germ. By Lemma 2.4.1](i) we have $H^{1}\left(\operatorname{gr}_{C}^{1} \mathscr{O}\right)=0$. From the standard exact sequence

$$
0 \longrightarrow \mathrm{J}_{C}^{(n+1)} \longrightarrow \mathrm{J}_{C}^{(n)} \longrightarrow \operatorname{gr}_{C}^{n} \mathscr{O} \longrightarrow 0 .
$$

we obtain the following easy but useful fact.

3.5.1. Lemma. The following assertions hold.

(i) If $H^{1}\left(\operatorname{gr}_{C}^{n} \mathscr{O}\right)=0$ and the map $H^{0}\left(\mathcal{J}_{C}^{(n)}\right) \rightarrow H^{0}\left(\operatorname{gr}_{C}^{n} \mathscr{O}\right)$ is surjective, then $H^{1}\left(\mathcal{J}_{C}^{(n+1)}\right) \simeq H^{1}\left(\mathcal{J}_{C}^{(n)}\right)$. In particular, $H^{1}(I)=0$ from the case $n=0$.

(ii) If for all $i<n$ one has $H^{1}\left(\operatorname{gr}_{C}^{i} \mathscr{O}\right)=0$ and the map $H^{0}\left(\mathcal{J}_{C}^{(i)}\right) \rightarrow$ $H^{0}\left(\operatorname{gr}_{C}^{i} \mathscr{O}\right)$ is surjective, then $H^{1}\left(\mathcal{J}_{C}^{(n)}\right) \simeq H^{1}\left(\operatorname{gr}_{C}^{n} \mathscr{O}\right)=0$.

(iii) If $H^{0}\left(\operatorname{gr}_{C}^{1} \mathscr{O}\right)=0$, then $H^{1}\left(\mathfrak{J}_{C}^{(2)}\right)=H^{1}\left(\operatorname{gr}_{C}^{2} \mathscr{O}\right)=0$.

In particular, if a general member $H \in\left|\mathscr{O}_{X}\right|$ is normal, then $H^{0}\left(\operatorname{gr}_{C}^{1} \mathscr{O}\right) \neq 0$.

Note however that this is necessary but not sufficient condition for normality of $H$ [MP11].

\subsection{Sheaves $\operatorname{gr}_{C}^{n} \omega$.}

3.6.1. Lemma. Let $f:(X, C) \rightarrow(Z, o)$ be an extremal curve germ.

(i) (Mor88, 1.2]) If $f$ is birational, then $R^{i} f_{*} \omega_{X}=0$ for $i>0$.

(ii) ([MP08a, Lemma 4.1]) If $f$ is a $\mathbb{Q}$-conic bundle and $Z$ is smooth, then there is a canonical isomorphism $R^{1} f_{*} \omega_{X} \simeq \omega_{Z}$. 
Proof. (i) follows from the Grauert-Riemenshneider vanishing. Let us prove (ii), Let $g: W \rightarrow X$ be a resolution. By [Kol86, Prop. 7.6] we have $R^{1}(f \circ g)_{*} \omega_{W}=\omega_{Z}$. Since $X$ has only terminal singularities, $g_{*} \omega_{W}=\omega_{X}$ and by the Grauert-Riemenshneider vanishing, $R^{i} g_{*} \omega_{W}=$ 0 for $i>0$. Then the Leray spectral sequence gives us $R^{1} f_{*} \omega_{X}=$ $R^{1}(f \circ g)_{*} \omega_{W}=\omega_{Z}$.

We also have the following useful fact

3.7. Corollary. Let $f:\left(X, C \simeq \mathbb{P}^{1}\right) \rightarrow(Z, o)$ be an extremal curve germ.

(i) If $f$ is birational, then $\operatorname{deg} \operatorname{gr}_{C}^{0} \omega=-1$.

(ii) Assume that $f$ is a $\mathbb{Q}$-conic bundle with smooth base. If $\operatorname{deg} \operatorname{gr}_{C}^{0} \omega \neq-1$, then $f^{-1}(o)=C$ (as a scheme).

Sketch of the proof. For [i) we note that by 3.6.][(i) for an arbitrary ideal $\mathscr{J}$ such that $\operatorname{Supp}\left(\mathscr{O}_{X} / \mathscr{J}\right) \subset C$ we have $\left.H^{1}\left(\boldsymbol{\omega}_{X} / \mathscr{J} \boldsymbol{\omega}_{X}\right)\right)=0$. Hence, $H^{1}\left(\operatorname{gr}_{C}^{0} \omega\right)=0$ in this case. On the other hand, $\operatorname{deg} \operatorname{gr}_{C}^{0} \omega<0$ by (3.1.2). For (ii) we apply [MP08a, Theorem 4.4] with $J=\mathcal{J}_{C}$.

3.7.1. Lemma ([Mor88, Cor. 1.15], Kol99, Prop. 4.2], [MP08a, Lemma 4.4.2]). Let $f:(X, C) \rightarrow(Z, o)$ be an extremal curve germ. Suppose that $C$ is reducible and let $P$ be a singular point of $C$. If $X$ is Gorenstein at $P$, then $f$ is a $\mathbb{Q}$-conic bundle and $C$ has two components meeting at $P$. If moreover $(Z, o)$ is smooth, then $X$ is Gorenstein (see 2.7.1](i)).

We will show below in 4.7.6 that in the above assumptions $(Z, o)$ is smooth automatically.

Proof. By Corollary 2.4.1 there are at least two components, say $C_{1}, C_{2} \subset C$ passing through $P$. Replacing $(X, C)$ with $\left(X, C_{1} \cup C_{2}\right)$ we may assume that $C=C_{1} \cup C_{2}$ (see Remark 2.4.2). Since the point $P \in X$ is Gorenstein, the sheaf $\operatorname{gr}_{C}^{0} \omega=\omega_{X} \otimes \mathscr{O}_{C}$ is invertible at $P$. Consider the injection

$$
\varphi: \operatorname{gr}_{C}^{0} \omega \hookrightarrow \operatorname{gr}_{C_{1}}^{0} \omega \oplus \operatorname{gr}_{C_{2}}^{0} \omega .
$$

Recall that $\left(X, C_{i}\right)$ is a (birational) extremal curve germ by Remark 2.4.2. Then by 3.4][i) we have $\operatorname{gr}_{C_{i}}^{0} \omega=\mathscr{O}_{C_{i}}(-1)$, so $H^{0}(\operatorname{Coker}(\varphi))=$ $H^{1}\left(\operatorname{gr}_{C}^{0} \boldsymbol{\omega}\right)$. On the other hand, $\operatorname{Coker}(\varphi)$ is a sheaf of finite length supported at $P$. Since $\operatorname{gr}_{C}^{0} \omega$ is invertible, $\operatorname{Coker}(\varphi)$ is non-trivial. So, $H^{1}\left(\operatorname{gr}_{C}^{0} \omega\right) \neq 0$ and by Corollary 3.7 the contraction $f$ is a $\mathbb{Q}$-conic bundle. Moreover, if the base $(Z, o)$ is smooth, then again by Corollary 3.7 we have $C=f^{-1}(o)$ (scheme-theoretically). Hence $P$ is the only singular point of $X$ and are done. 


\section{TOPOLOGicAl OBSERVATIONS}

Let $\mathrm{Cl}^{\mathrm{sc}}(X)$ be the subgroup of the divisor class group $\mathrm{Cl}(X)$ consisting of Weil divisor classes which are $\mathbb{Q}$-Cartier. We will use the following easy consequence of the classification of terminal singularities without additional reference.

4.1. Proposition ([Kaw88, Lemma 5.1]). Let $(X, P)$ be an (analytic) germ of three-dimensional terminal singularity of index $m$. Then

$$
\mathrm{Cl}^{\mathrm{sc}}(X, P) \simeq \pi_{1}(X \backslash\{P\}) \simeq \mathbb{Z} / m \mathbb{Z} .
$$

4.2. Definition (Mor88, (0.4.16), (1.7)]). Let $(X, P)$ be a terminal three-dimensional singularity of index $m$ and let $C \subset X$ be a smooth curve passing through $P$. We say that $C$ is (locally) primitive at $P$ if the natural map

$$
\varrho: \mathbb{Z} \simeq \pi_{1}(C \backslash\{P\}) \longrightarrow \pi_{1}(X \backslash\{P\}) \simeq \mathbb{Z} / m \mathbb{Z}
$$

is surjective and imprimitive at $P$ otherwise. The order $s$ of Coker $(\varrho)$ is called the splitting degree and the number $\bar{m}=\mathrm{m} / \mathrm{s}$ is called the subindex of $P \in C$.

It is easy to see that the splitting degree coincides with the number of irreducible components of the preimage $C^{\sharp}$ of $C$ under the index-one cover $X^{\sharp} \rightarrow X$ near $P$. If $P$ is primitive, we put $s=1$ and $\bar{m}=m$.

4.3. In the above notation it is easy to show that for any Weil divisor class $\xi \in \mathrm{Cl}^{\mathrm{sc}}(X, P)$ there exists an effective Weil $\mathbb{Q}$-Cartier divisor $D$ whose class in $\mathrm{Cl}^{\text {sc }}(X, P)$ equals $\xi$ and such that $D \cap C=\{P\}$. Then one can define the intersection number $\xi \cdot C:=(D \cdot C)_{P} \bmod \mathbb{Z}$. Hence there exists a well-defined homomorphism

$$
\mathrm{cl}: \mathrm{Cl}^{\mathrm{sc}}(X, P) \longrightarrow \frac{1}{m} \mathbb{Z} / \mathbb{Z} \subset \mathbb{Q} / \mathbb{Z}, \quad \xi \longmapsto \xi \cdot C .
$$

The curve $C$ is locally primitive at $P$ if and only if the map cl is an isomorphism. In general, the splitting degree equals the order of the kernel of cl [Mor88, 1.7].

4.4. Let $(X, C)$ be an extremal curve germ with irreducible central fiber $C$. Let $P_{1}, \ldots, P_{n}$ be all the non-Gorenstein points of $X$ and let $m_{1}, \ldots, m_{n}$ be their indices. We have the following exact sequence

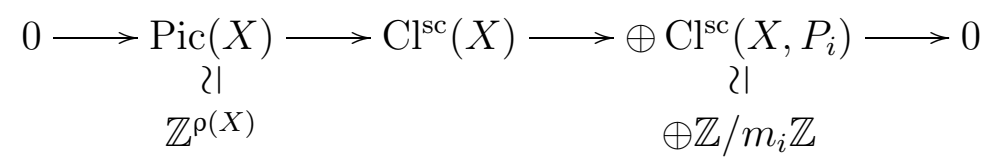

4.4.2. Corollary. In the above notation assume that $C$ is irreducible. Let $D_{i}, n=1, \ldots, n$ be an effective Weil $\mathbb{Q}$-Cartier divisor whose class generates $\mathrm{Cl}^{\mathrm{sc}}\left(X, P_{i}\right)$ and let $H$ be an effective Cartier divisor such that $H \cdot C=1$. Then the following holds. 
(i) The group $\mathrm{Cl}^{\mathrm{sc}}(X)$ is generated by the classes of $H, D_{1}, \ldots, D_{n}$.

(ii) If the point $P_{i}$ is imprimitive of splitting degree $s_{i}$ and subindex $\bar{m}_{i}$, then the class of $H-\bar{m}_{i} D_{i}$ is an $s_{i}$-torsion element in $\mathrm{Cl}^{\mathrm{sc}}(X)$.

(iii) If $(X, C)$ is locally primitive at distinct points $P_{i}, P_{j} \in C$ and $\operatorname{gcd}\left(m_{i}, m_{j}\right)=d \neq 1$, then the class of $\frac{m_{i}}{d} D_{i}-\frac{m_{j}}{d} D_{j}$ is a $d$ torsion element in $\mathrm{Cl}^{\mathrm{sc}}(X)$.

4.5. Construction. Let $f:(X, C) \rightarrow(Z, o)$ be an extremal curve germ and let $\theta:\left(X^{b}, C^{b}\right) \rightarrow(X, C)$ be a finite cover which is étale in codimension one. Clearly, $\theta$ must be étale over the Gorenstein locus of $X$. The Stein factorization gives us the following diagram.

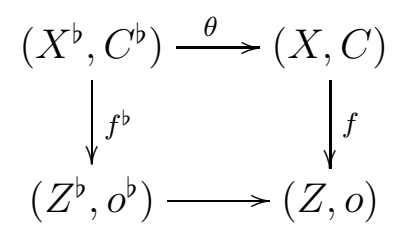

where $\left(Z^{b}, o^{b}\right) \rightarrow(Z, o)$ is a finite cover which is étale over $Z \backslash\{o\}$. We have $K_{X^{b}}=\theta^{*} K_{X}$ and singularities of $X^{b}$ are terminal. In particular, $\left(X^{b}, C^{b}\right)$ is an extremal curve germ. Note that in our situation $X^{b}$ is the normalization of $X \times_{Z} Z^{b}$ and $C^{b}:=f^{b-1}(C)_{\text {red }}$.

Conversely, if $f:(X, C) \rightarrow(Z, o)$ is an extremal curve germ and $\left(Z^{b}, o^{b}\right) \rightarrow(Z, o)$ is a finite cover which is étale over $Z \backslash\{o\}$. Then the base change produces the diagram (4.5.1), where $X^{b}$ is the normalization of $X \times_{Z} Z^{b},\left(X^{b}, C^{b}\right)$ is an extremal curve germ, and $\theta$ is étale in codimension one.

4.6. Definition. Let $(X, C)$ be an extremal curve germ. By the above construction 4.5 the torsion part $\mathrm{Cl}(X)_{\text {tors }} \subset \mathrm{Cl}(X)$ defines an abelian Galois cover

$$
\tau:\left(X^{\prime}, C^{\prime}\right) \longrightarrow(X, C)
$$

which is étale over the Gorenstein locus of $X$. We call this map the torsion free cover of $(X, C)$ and the degree of this cover we call the topological index of $(X, C)$. Similar to (4.5.1) we have the diagram

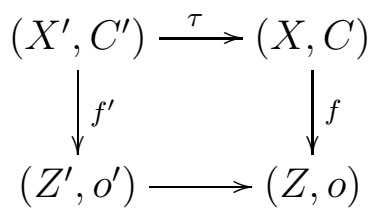

Hence $\left(X^{\prime}, C^{\prime}\right)$ is also an extremal curve germ. Clearly, $\mathrm{Cl}\left(X^{\prime}\right)$ is torsion free. 
4.6.3. Lemma. Let $(X, C)$ be an extremal curve germ and let $\theta$ : $\left(X^{b}, C^{b}\right) \rightarrow(X, C)$ be a finite cover which is étale in codimension two. Then $\theta$ is a cyclic cover.

Proof. We may assume that the cover $\theta$ is Galois with group $G$ and it is sufficient to show that the group $G$ is cyclic. By taking composition with the torsion free cover, we may assume also that $\mathrm{Cl}^{\mathrm{sc}}\left(X^{b}\right)$ is torsion free. By the construction $G$ effectively acts on $C^{b}=\cup C_{i}^{b}$ (because $X$ has only isolated singularities). Since $C^{b}$ is a tree of smooth rational curves, it is easy to prove by induction on the number of components of $C^{b}$ that $G$ has either an invariant component $C_{i}^{b} \subset C^{b}$ or a fixed point $P^{b} \in \operatorname{Sing}\left(C^{b}\right)$.

In the latter case, let $P=\theta\left(P^{b}\right)$. There is a surjection $\pi_{1}(X \backslash\{P\}) \rightarrow$ $G$. Since $\pi_{1}(U \backslash\{P\})$ is cyclic (see 4.1), we are done.

In the former case, let $C_{i}:=\theta\left(C_{i}^{b}\right)$. By Remark 2.4.2 we may replace $\left(X^{b}, C^{b}\right)$ with $\left(X^{b}, C_{i}^{b}\right)$ and $(X, C)$ with $\left(X, C_{i}\right)$. Thus $C=C_{i}, C^{b}=C_{i}^{b}$, and $C^{b} / G=C \simeq \mathbb{P}^{1}$. Assume that the group $G$ is not cyclic. Then there is no fixed points on $C^{b}$. If $X^{b}$ has a point of index $m>1$, then its orbit contains at least two points of the same index. By (4.4.1) the torsion part of the group $\mathrm{Cl}^{\mathrm{sc}}\left(X^{\mathrm{b}}\right)$ is non-trivial. This contradicts the assumption above. Thus $X^{b}$ is Gorenstein.

Let $P_{1}, \ldots, P_{n} \in C$ be all branch points of $C^{b} \rightarrow C$ and let $m_{1}, \ldots, m_{n}$ be their ramification indices. By the Hurwitz formula we can write

$$
\frac{1}{|G|}\left(2 g\left(C_{i}^{b}\right)-2\right)=2 g\left(C_{i}\right)-2+\sum_{i=1}^{n}\left(1-\frac{1}{m_{i}}\right)
$$

Hence, $\sum 1 / m_{i}>n-2$. Since the group $G$ is not cyclic, we have $n>2$. The index of the point $P_{i} \in X$ is equal to $m_{i}$. By (3.1.1) and Lemma 3.4 we have $w_{P_{i}}(0) \geq 1 / m_{i}$ and $i_{P_{i}}(1) \geq 1$. Therefore, $\sum w_{P_{i}}(0)>1$ and $\operatorname{deg} \operatorname{gr}_{C}^{0} \omega=-1$ by (3.1.5). Then we get a contradiction by (3.1.2).

4.6.4. Corollary. Let $(X, C)$ be an extremal curve germ. Then the torsion part $\mathrm{Cl}(X)_{\text {tors }} \subset \mathrm{Cl}(X)$ is a cyclic group. Hence the torsion free cover (4.6.1) is cyclic. Moreover, $X^{\prime}, C^{\prime}$ ) has no finite cover which is étale in codimension one.

4.6.5. Corollary ([Pro97, Lemma 1.10]). If $f:(X, C) \rightarrow(Z, o)$ is a $\mathbb{Q}$-conic bundle germ, then $(Z, o)$ is a cyclic quotient singularity.

Proof. Follows from Lemma 4.6 .3 and 4.5 ,

4.7. From now on we assume that $f:(X, C) \rightarrow(Z, o)$ is an extremal curve germ with $C \simeq \mathbb{P}^{1}$. Assume that the torsion part $\operatorname{Cl}(X)_{\text {tors }}=$ $\mathbb{Z} / d \mathbb{Z}$ is non-trivial and consider the torsion free cover (4.6.1). Thus $(X, C)=\left(X^{\prime}, C^{\prime}\right) / G$ and $(Z, o)=\left(Z^{\prime}, o^{\prime}\right) / G$, where $G=\boldsymbol{\mu}_{d}$ acts on $Z^{\prime} \backslash$ 
$\left\{o^{\prime}\right\}$ and $X^{\prime} \backslash \tau^{-1}(\operatorname{Sing}(X))$ freely. We distinguish two cases (cf. Mor88, $(1.12)])$ :

4.7.1. Case: $C^{\prime}$ is irreducible. Then $G=\boldsymbol{\mu}_{d}$ has exactly two fixed points $P_{1}^{\prime}$ and $P_{2}^{\prime}$ on $C^{\prime} \simeq \mathbb{P}^{1}$. They give us two points $P_{i}:=\tau\left(P_{i}^{\prime}\right)$ on $C$ whose indices are divisible by $d$. The germ $(X, C)$ is locally primitive along $C$.

4.7.2. Case: $C^{\prime}=\cup_{i=1}^{s} C_{i}^{\prime}$, where $s>1$ and $C_{i}^{\prime} \simeq \mathbb{P}^{1}$. In this case, $G$ acts on $\left\{C_{1}^{\prime}, \ldots, C_{s}^{\prime}\right\}$ transitively. Since $p_{\mathrm{a}}\left(C^{\prime}\right)=0$, each component $C_{i}^{\prime}$ meets the closure of $C^{\prime} \backslash C_{i}^{\prime}$ at one point. Therefore, in this case, all the irreducible components $C_{i}^{\prime}$ pass through one point $P^{\prime}$ and do not meet each other elsewhere. In this case $(X, C)$ is imprimitive at $\tau\left(P^{\prime}\right)$ of splitting degree $s$ and has no other locally imprimitive points.

It is worthwhile to mention in the case 4.7 .2 that $\tau\left(P^{\prime}\right)$ is the only non-Gorenstein point of $X$ and $d=s$ (see [Mor88, Th. 6.7, 9.4] and [MP08a, § 7]).

4.7.3. Corollary ([Mor88, $(1.10)])$. Let $\left(X, C \simeq \mathbb{P}^{1}\right)$ be an extremal curve germ. Let $P_{1}, \ldots, P_{n}$ be all the non-Gorenstein points of $X$. The following are equivalent:

(i) $D \cdot C=1 / m_{1} \cdots m_{n}$ for some $D \in \mathrm{Cl}^{\mathrm{sc}}(X)$,

(ii) $\mathrm{Cl}^{\mathrm{sc}}(X) \simeq \mathbb{Z}$,

(iii) $\mathrm{Cl}^{\mathrm{sc}}(X)$ is torsion-free,

(iv) $(X, C)$ is locally primitive and $\operatorname{gcd}\left(m_{i}, m_{j}\right)=1, i \neq j$.

Proof. Follows from Lemma 4.6.3 and (4.4.1).

4.7.4. Corollary (cf. [MP08a, Lemma 2.8]). Let $\left(X, C \simeq \mathbb{P}^{1}\right)$ be an extremal curve germ. Let $d$ be the topological index of $(X, C)$ and let $m_{1}, \ldots, m_{r}$ be indices of all the non-Gorenstein points. Assume that $(X, C)$ is either divisorial or a $\mathbb{Q}$-conic bundle which is not toroidal 2.3 . Then

$$
-K_{X} \cdot C=d / m_{1} \cdots m_{r}
$$

Proof. It follows from (4.4.1) that for the ample generator $D$ of the group $\mathrm{Cl}^{\mathrm{sc}}(X) / \equiv$ one has $D \cdot C=d / m_{1} \cdots m_{r}$. Write $-K_{X} \equiv a D$ for some $a \in \mathbb{Z}$. Intersecting $D$ and $K_{X}$ with a general one-dimensional fiber $L$, we obtain $-K_{X} \cdot L=D \cdot L$ and $a=1$.

Now we can strengthen the assertion of Lemma 3.7.1.

4.7.6. Corollary. Let $f:(X, C) \rightarrow(Z, o)$ be an extremal curve germ. Suppose that $C$ is reducible and let $P$ be a singular point of $C$. If $X$ is Gorenstein at $P$, then $(Z, o)$ is smooth and $f$ is a Gorenstein conic bundle. 
Proof. By Lemma 3.7.1 $f$ is a $\mathbb{Q}$-conic bundle and $(Z, o)$ is singular. Recall (see Lemma 2.5) that $(Z, o)$ is a quotient singularity. Thus there is a finite Galois étale over $Z \backslash\{o\}$ cover $\left(Z^{b}, o^{b}\right) \rightarrow(Z, o)$ where $\left(Z^{b}, o^{b}\right)$ is smooth. Then we can consider the base change (see (4.5.1)). Thus $X=X^{b} / G$, where $G$ is a finite group acting on $X^{b}$ freely outside finite number of points. Since $X$ is Gorenstein at $P$, so is $X^{b}$ at all the points $P_{i}^{b} \in \theta^{-1}(P)$. Moreover, $\theta$ is étale over $P$ by (4.1.1). Hence, the central curve $C^{b}$ is singular at $P_{i}^{b}$. By Lemma 3.7.1 the variety $X^{b}$ is Gorenstein and by Corollary 2.7.1 the contraction $f^{b}: X^{b} \rightarrow Z^{b}$ is a standard Gorenstein conic bundle. In particular, $C^{b}$ is a plane conic. Thus $C^{b}$ has two components meeting at one point $\theta^{-1}(P)$ which must be fixed by $G$. Again by (4.1.1) the group $G$ is trivial, a contradiction.

4.7.7. Corollary. Let $f:\left(X, C \simeq \mathbb{P}^{1}\right) \rightarrow(Z, o)$ be an extremal curve germ. Assume that $(X, C)$ is locally imprimitive at $P$. If the subindex of $P$ equals 1 , then $f$ is a $\mathbb{Q}$-conic bundle and in the diagram (4.6.2) the contraction $f^{\prime}$ is a Gorenstein conic bundle.

$\mathbb{Q}$-conic bundles which are quotients of Gorenstein conic bundles by a finite group were described in [Pro97, § 2]. It turns out that such a $\mathbb{Q}$-conic bundle is locally imprimitive if and only if it is of type 9.1.2.

4.7.8. Corollary (cf. [Mor88, Prop. 1.14]). Let $f:(X, C) \rightarrow(Z, o)$ be an extremal curve germ. Assume that $C$ is irreducible. If $\mathrm{gr}_{C}^{0} \boldsymbol{\omega} \neq$ $\mathscr{O}_{C}(-1)$, then $f$ is a $\mathbb{Q}$-conic bundle and in notation of (4.6.2) we have $f^{\prime-1}\left(o^{\prime}\right)=C^{\prime}$. If furthermore $(X, C)$ is locally primitive, then it is toroidal (see 2.3).

Proof. By Remark 3.1.6 the contraction $f$ is a $\mathbb{Q}$-conic bundle. Apply the construction (4.6.2). Since

$$
H^{1}\left(\operatorname{gr}_{C}^{0} \omega\right)=H^{1}\left(\operatorname{gr}_{C^{\prime}}^{0} \omega\right)^{\boldsymbol{\mu}_{d}}
$$

we have $H^{1}\left(\operatorname{gr}_{C^{\prime}}^{0} \omega\right) \neq 0$. By Corollary $3.7 C^{\prime}=f^{\prime-1}\left(o^{\prime}\right)$. If $f$ is locally primitive, $C^{\prime}$ is irreducible (see 4.7.1). So $C^{\prime} \simeq \mathbb{P}^{1}$ and $X^{\prime}$ is smooth. Up to analytic isomorphism we may assume that there exists a $\boldsymbol{\mu}_{d^{-}}$ equivariant decomposition $X^{\prime} \simeq Z^{\prime} \times \mathbb{P}^{1}$. So, $f$ is toroidal 2.3, Pro97, $\S 2]$.

4.8. Proposition ([MP08a, Lemma 9.2.3], [Mor88, 0.4.13.3]). An extremal curve germ $\left(X, C \simeq \mathbb{P}^{1}\right)$ has at most two non-Gorenstein points.

Proof. Assume that $P_{1}, P_{2}, P_{3} \in X$ are singular points of indices $m_{1}$, $m_{2}, m_{3}>1$. If $(X, C)$ is locally imprimitive at some point, then the torsion free cover $\tau:\left(X^{\prime}, C^{\prime}\right) \rightarrow(X, C)$ has the form 4.7.2, i.e. $C^{\prime}$ is a union of $s$ components $C_{1}^{\prime}, \ldots, C_{s}^{\prime}$ passing through one point, say $P^{\prime}$, and $\tau$ is étale over $X^{\prime} \backslash\left\{P^{\prime}\right\}$. By Corollary 4.7.7 the point $P^{\prime} \in X^{\prime}$ is 
not Gorenstein. Thus for any component $C_{i}^{\prime}$ the germ $\left(X, C_{i}^{\prime}\right)$ has at least three non-Gorenstein points.

Replacing $(X, C)$ with $\left(X^{\prime}, C_{i}^{\prime}\right)$ we may assume that $(X, C)$ is locally primitive, i.e. the maps $\pi_{1}\left(C \backslash\left\{P_{i}\right\}\right) \rightarrow \pi_{1}\left(U_{i} \backslash\left\{P_{i}\right\}\right)$ are surjective, where $U_{i} \subset X$ is a small neighborhood of $P_{i}$. Then using Van Kampen's theorem and (4.1.1) it is easy to compute the fundamental group of $X \backslash\left\{P_{1}, P_{2}, P_{3}\right\}$ :

$\pi_{1}\left(X \backslash\left\{P_{1}, P_{2}, P_{3}\right\}\right)=\left\langle\sigma_{1}, \sigma_{2}, \sigma_{3}\right\rangle /\left\{\sigma_{1}^{m_{1}}=\sigma_{2}^{m_{2}}=\sigma_{3}^{m_{3}}=\sigma_{1} \sigma_{2} \sigma_{3}=1\right\}$.

This group has a finite quotient group $G$ in which the images of $\sigma_{1}, \sigma_{2}$, $\sigma_{3}$ are exactly of order $m_{1}, m_{2}$ and $m_{3}$, respectively (see, e.g., [Feu71]). The above quotient defines a finite Galois cover $\tau:\left(X^{\prime}, C^{\prime}\right) \rightarrow(X, C)$ with non-abelian Galois group $G$. This contradicts Lemma 4.6.3,

\section{LOCAL DESCRIPTION}

5.1. Notation. Let $(X, P)$ be a threefold terminal singularity of index $m$ and let $C \subset(X, P)$ be a smooth curve such that $P$ has subindex $\bar{m}$ and splitting degree $s$ (see 4.2). We use the notation of 2.1. Put $C^{\sharp}:=\pi^{-1}(C)$. Then $C^{\sharp}$ has $s$ irreducible components.

Let $\left(C^{\dagger}, P^{\dagger}\right)$ be the normalization of an irreducible component $C^{\sharp}(i) \subset$ $C^{\sharp}, 1 \leq i \leq s$ and let $\tau:\left(X^{\prime}, C^{\prime}\right) \rightarrow(X, C)$ be the torsion free cover (see (4.6.2) ). Then $\boldsymbol{\mu}_{m}$ naturally acts on $\left(X^{\sharp}, P^{\sharp}\right)$ and $\left(C^{\sharp}, P^{\sharp}\right)$, and so does $\boldsymbol{\mu}_{\bar{m}}$ on $\left(C^{\prime}, P^{\prime}\right)$. Let

$$
\eta: \mathscr{O}_{X^{\sharp}, P^{\sharp}} \longrightarrow \mathscr{O}_{X^{\dagger}, P^{\dagger}}
$$

be the natural map. Since $(X, P)$ and $(C, P)$ are normal, one has

$$
\mathscr{O}_{X, P}=\left(\mathscr{O}_{X^{\sharp}, P^{\sharp}}\right)^{\boldsymbol{\mu}_{m}} \quad \text { and } \quad \mathscr{O}_{C, P}=\left(\mathscr{O}_{C^{\dagger}, P^{\dagger}}\right)^{\boldsymbol{\mu}_{\bar{m}}} \text {. }
$$

Since $\boldsymbol{\mu}_{m}$ acts freely on $X^{\sharp} \backslash\left\{P^{\sharp}\right\}$, so it does on $C^{\sharp} \backslash\left\{P^{\sharp}\right\}$ and hence $\boldsymbol{\mu}_{\bar{m}}$ on $C^{\dagger} \backslash\left\{P^{\dagger}\right\}$. Hence $\mathscr{O}_{C^{\dagger}, P^{\dagger}}$ has a uniformizing parameter, say $t$, such that $t$ is a $\boldsymbol{\mu}_{\bar{m}}$-semi-invariant. Let $\chi$ be a generator of $\operatorname{Hom}\left(\boldsymbol{\mu}_{m}, \mathbb{C}^{*}\right)=$ $\mathbb{Z} / m \mathbb{Z}$ whose restriction $\bar{\chi}$ to $\boldsymbol{\mu}_{\bar{m}}$ is the character associated to $t$. Then $\mathscr{O}_{C, P}=\mathbb{C}\{t\}^{\boldsymbol{\mu}_{\bar{m}}}$.

For a semi-invariant $z \neq 0$, let $C^{\sharp}-\operatorname{wt}(z)$ (or simply wt $(z)$ if there is no confusion) be $n \in \mathbb{Z} / m \mathbb{Z}$ such that $n \chi$ is the character associated to $z$. For a $\boldsymbol{\mu}_{m}$-semi-invariant $z \in \mathscr{O}_{X^{\sharp}, P^{\sharp}}$, let

$$
C^{\sharp}-\operatorname{ord}(z):=\sup \left\{n \in \mathbb{Z}_{\geq 0} \mid \eta(z) \in t^{n} \mathbb{C}\{t\}\right\} .
$$

We also write $\operatorname{ord}(z)$, if it does not cause confusion. Let

$$
\operatorname{ow}(z):=(\operatorname{ord}(z), \operatorname{wt}(z)) \text {. }
$$

We define semigroups

$$
\begin{aligned}
\operatorname{ord}\left(C^{\sharp}\right):=\left\{\operatorname{ord}(z) \mid z \in \mathscr{O}_{C^{\sharp}, P^{\sharp}}, z \neq 0\right\} \subset \mathbb{Z}_{>0}, \\
\operatorname{ow}\left(C^{\sharp}\right):=\left\{(\operatorname{ord}(z), \operatorname{wt}(z)) \mid z \in \mathscr{O}_{C^{\sharp}, P^{\sharp}}, z \neq 0\right\} \subset \mathbb{Z}_{\geq 0} \times \mathbb{Z} / m \mathbb{Z} .
\end{aligned}
$$


One can show that in some coordinates $C^{\sharp}$ can be given by a monomial parametrization (see [Mor88, Lemma 2.7] for the precise statement).

5.2. Notation. Let $f:\left(X, C \simeq \mathbb{P}^{1}\right) \rightarrow(Z, o)$ be an extremal curve germ and let $P \in C$ be a point of index $m \geq 1$. Let $s$ and $\bar{m}$ be the splitting degree and subindex, respectively. Consider the index-one $\boldsymbol{\mu}_{m^{-}}$ cover $\pi:\left(X^{\sharp}, P^{\sharp}\right) \rightarrow(X, P)$ and let $C^{\sharp}:=\pi^{-1}(C)$. Take normalized $\ell$-coordinates $\left(x_{1}, \ldots, x_{4}\right)$ and let $\phi$ be an $\ell$-equation of $X \supset C \ni P$ (see [Mor88, 2.6]). Put $a_{i}:=\operatorname{ord}\left(x_{i}\right)$. Note that $a_{i}<\infty$ and $\operatorname{wt}\left(x_{i}\right) \equiv a_{i}$ $\bmod \bar{m}$.

The following is the key fact in the local classification of possible singularities of extremal curve germs.

5.3. Lemma ([Mor88, 3.8, 4.2], MP08a, § 5]). In the above notation, assume that $P$ is not of type $\left(\mathrm{IE}^{\vee}\right)$ below. Then $\mathrm{ow}\left(C^{\sharp}\right)$ is generated by ow $\left(x_{1}\right)$ and ow $\left(x_{2}\right)$. In particular, $C^{\sharp}$ is a planar curve.

This lemma allows to obtain a local classification of possible singularities. We reproduce this classification below. We start with the primitive case.

5.4. Proposition ([Mor88, Prop. 4.2], [MP08a, Prop. 5.2.1]). Let $f$ : $\left(X, C \simeq \mathbb{P}^{1}\right) \rightarrow(Z, o)$ be an extremal curve germ and let $P \in C$ be a primitive point of index $m \geq 1$. Then modulo permutations of $x_{i}$ 's, the semigroup $\operatorname{ord}\left(C^{\sharp}\right)$ is generated by $a_{1}$ and $a_{2}$. Moreover, exactly one of the following holds:

(IA) $a_{1}+a_{3} \equiv 0 \bmod m, a_{4}=m, m \in \mathbb{Z}_{>0} a_{1}+\mathbb{Z}_{>0} a_{2}$, where we may still permute $x_{1}$ and $x_{3}$ if $a_{2}=1$,

(IB) $a_{1}+a_{3} \equiv 0 \bmod m, a_{2}=m, a_{1} \geq 2$,

(IC) $a_{1}+a_{2}=a_{3}=m, a_{4} \not \equiv a_{1}, a_{2} \bmod m, 2 \leq a_{1}<a_{2}, m \geq 5$,

(IIA) $m=4, P$ is of type $\mathrm{cAx} / 4$, and $\operatorname{ord}(x)=(1,1,3,2)$,

(IIB) $m=4, P$ is of type $\mathrm{cAx} / 4$, and $\operatorname{ord}(x)=(3,2,5,5)$,

(III) $m=1, X=X^{\sharp}, C=C^{\sharp}$, and $P \in X$ is a cDV point.

Now consider the locally imprimitive case.

5.5. Proposition ([Mor88, Prop. 4.2], [MP08a, Prop. 5.3.1]). Let $f$ : $\left(X, C \simeq \mathbb{P}^{1}\right) \rightarrow(Z, o)$ be an extremal curve germ and let $P \in C$ be an imprimitive point of index $m$.

Modulo permutations of $x_{i}$ 's and changes of $\ell$-characters, the semigroup ow $\left(C^{\sharp}\right)$ is generated by ow $\left(x_{1}\right)$ and ow $\left(x_{2}\right)$ except for the case $\left(\mathrm{IE}^{\vee}\right)$ below. Moreover, exactly one of the following holds:

$\left(\mathrm{IA}^{\vee}\right) \bar{m}>1, \operatorname{wt}\left(x_{1}\right)+\operatorname{wt}\left(x_{3}\right) \equiv 0 \bmod m$, ow $x_{4}=(\bar{m}, 0)$, ow $\left(C^{\sharp}\right)$ is generated by ow $\left(x_{1}\right)$ and ow $\left(x_{2}\right)$, and $w_{P}(0) \geq 1 / 2$. 
$\left(\mathrm{IC}^{\vee}\right) s=2, \bar{m}$ is an even integer $\geq 4$, and

$$
\begin{array}{cccccc} 
& x_{1} & x_{2} & x_{3} & x_{4} & \\
\text { wt } & 1 & -1 & 0 & \bar{m}+1 & \bmod m \\
\text { ord } & 1 & \bar{m}-1 & \bar{m} & \bar{m}+1 &
\end{array}
$$

$\left(\mathrm{II}^{\vee}\right) \bar{m}=s=2, P$ is of type $\mathrm{cAx} / 4$, and

$\begin{array}{cccccc} & x_{1} & x_{2} & x_{3} & x_{4} & \\ \text { wt } & 1 & 3 & 3 & 2 & \bmod 4 \\ \text { ord } & 1 & 1 & 1 & 2 & \end{array}$

$\left(\mathrm{ID}^{\vee}\right) \bar{m}=1, s=2, P$ is of type $\mathrm{cA} / 2$ or $\mathrm{cAx} / 2$, and

$\begin{array}{ccccc} & x_{1} & x_{2} & x_{3} & x_{4} \\ \text { wt } & 1 & 1 & 1 & 0 \\ \text { ord } & 1 & 1 & 1 & 1\end{array}$

$\bmod 2$

$\left(\mathrm{IE}^{\vee}\right) \bar{m}=2, s=4, P$ is of type $\mathrm{cA} / 8$, and

$$
\begin{array}{cccccc} 
& x_{1} & x_{2} & x_{3} & x_{4} & \\
\text { wt } & 5 & 1 & 3 & 0 & \bmod 8 \\
\text { ord } & 1 & 1 & 1 & 2 &
\end{array}
$$

Moreover, cases $\left(\mathrm{ID}^{\vee}\right)$ and $\left(\mathrm{IE}^{\vee}\right)$ occurs if and only if $f$ is a $\mathbb{Q}$-conic bundle and $C^{\prime}=f^{\prime-1}\left(o^{\prime}\right)$. In these cases, $P$ is the only non-Gorenstein point.

Proofs are based on very careful local computations. We do not present them here. See Example 5.8 for sample of computations.

5.5.1. Corollary. Let $\left(X, C \simeq \mathbb{P}^{1}\right)$ be an extremal curve germ. Assume that $\operatorname{gr}_{C}^{0} \omega \neq \mathscr{O}(-1)$. Then $(X, C)$ is a $\mathbb{Q}$-conic bundle germ which is either toroidal or the only non-Gorenstein point of $(X, C)$ is of type $\left(\mathrm{ID}^{\vee}\right)$.

Proof. By Corollary 4.7.8 $(X, C)$ is $\mathbb{Q}$-conic bundle and $C^{\prime}=f^{\prime-1}\left(o^{\prime}\right)$. Assume that it is not a toroidal. Then again by 4.7.8 it is locally imprimitive and by the proposition above $(X, C)$ has a unique nonGorenstein point, say $P$, which is of type $\left(\mathrm{ID}^{\vee}\right)$ or $\left(\mathrm{IE}^{\vee}\right)$. In the case $\left(\mathrm{IE}^{\vee}\right)$ we have $-K_{X} \cdot C=1 / 2$ (see 4.7.4). Easy computations show that $w_{P}(0)=1 / 2$ and so $\operatorname{deg} \operatorname{gr}_{C}^{0} \omega=-1$ by (3.1.2).

5.6. By Lemma 5.3 there exist monomials $\lambda_{3}$ and $\lambda_{4}$ in $x_{1}, x_{2}$ such that $x_{3}=\lambda_{3}\left(x_{1}, x_{2}\right)$ and $x_{4}=\lambda_{4}\left(x_{1}, x_{2}\right)$ on $C^{\sharp}$. Then

$$
x_{1}^{s a_{2}}-x_{2}^{s a_{1}}, \quad x_{3}-\lambda_{3}, \quad x_{4}-\lambda_{4}
$$

generate the defining ideal $I^{\sharp}$ of $C^{\sharp} \subset \mathbb{C}^{4}$. Then the equation of $X^{\sharp}$ can be written as follows

$$
\phi=\left(x_{1}^{s a_{2}}-x_{2}^{s a_{1}}\right) \phi_{2}+\left(x_{3}-\lambda_{3}\right) \phi_{3}+\left(x_{4}-\lambda_{4}\right) \phi_{4}
$$

for some semi-invariant $\phi_{i} \in \mathbb{C}\left\{x_{1}, \ldots, x_{4}\right\}$ with suitable weights. 
5.7. Lemma. Under the notation of 5.4 and 5.5, one has

(i) if $P$ is of type (IC) or $\left(\mathrm{IC}^{\vee}\right)$, then $\left(X^{\sharp}, P^{\sharp}\right)$ is smooth and $I^{\sharp}=$ $\left(x_{1}^{s a_{2}}-x_{2}^{s a_{1}}, x_{4}-\lambda_{4}, \phi\right)$

(ii) if $P$ is of type (IIB) or (II $\left.{ }^{\vee}\right)$, then $I^{\sharp}=\left(x_{3}-\lambda_{3}, x_{4}-\lambda_{4}, \phi\right)$.

Proof. Let us consider for example the case (IC). Then $\lambda_{3}$ must be $x_{1} x_{2}$, and so

$$
\phi=\left(x_{1}^{s a_{2}}-x_{2}^{s a_{1}}\right) \phi_{2}+\left(x_{3}-x_{1} x_{2}\right) \phi_{3}+\left(x_{4}-\lambda_{4}\right) \phi_{4} .
$$

Since $P$ is of type (IC), one sees that $m=a_{1}+a_{2}>4, \phi_{2} \in(x)$, and that $\phi_{4}, \lambda_{4} \in(x)^{2}$ because $\operatorname{wt}\left(x_{4}\right) \not \equiv 0, \pm \operatorname{wt}\left(x_{1}\right), \pm \operatorname{wt}\left(x_{2}\right) \bmod m$. Since $m \geq 5$, by the classification of terminal singularities either $x_{1} x_{2}$ or $x_{3}$ must appear in the power series expansion. Since $a_{1}, a_{2} \geq 2$, this is only possible if $\phi_{3}$ is a unit.

5.8. Example. According to Lemma [5.7](i)] a point $P \in(X, C)$ of type $\left(\mathrm{IC}^{\vee}\right)$ can be written as follows:

$$
(X, C, P)=\left(\mathbb{C}_{x_{1}, x_{2}, x_{4}}^{3},\left\{x_{4}=x_{2}^{2}-x_{1}^{2 \bar{m}-2}=0\right\}, 0\right) / \boldsymbol{\mu}_{2 \bar{m}}(1,-1, \bar{m}+1)
$$

We have $C \simeq\left\{x_{4}=x_{2}-x_{1}^{\bar{m}-1}=0\right\} / \boldsymbol{\mu}_{\bar{m}}$ and a local uniformizing parameter on $C$ is $x_{1}^{\bar{m}}$. Hence, $\mathscr{O}_{C, P}=\mathbb{C}\left\{x_{1}^{\bar{m}}\right\}$. Furthermore,

$$
\begin{aligned}
\mathscr{O}_{C}\left(m K_{X}\right) & =\mathscr{O}_{C}\left(d x_{1} \wedge d x_{2} \wedge d x_{4}\right)^{m}, \\
\operatorname{gr}_{C}^{0} \omega & =\mathscr{O}_{C}\left(x_{1}^{\bar{m}-1} d x_{1} \wedge d x_{2} \wedge d x_{4}\right), \\
\operatorname{gr}_{C}^{1} \mathscr{O} & =\mathscr{O}_{C}\left(x_{1}^{\bar{m}-1} x_{4}\right) \oplus \mathscr{O}_{C}\left(x_{1}^{2}\left(x_{2}^{2}-x_{1}^{2 \bar{m}-2}\right)\right), \\
w_{P}(0) & =(\bar{m}-1) / \bar{m}, \quad i_{p}(1)=2 .
\end{aligned}
$$

\section{Deformations}

In this section we discuss deformations of extremal curve germs. It is known that a small deformation of a terminal singularity is again terminal (see e.g. [Ish14, Theorem 9.1.14]). Moreover, any three-dimensional terminal singularity admits a $\mathbb{Q}$-smoothing, i.e. a deformation to a collection of cyclic quotient singularities [Rei87, 6.4A].

To study extremal curve germs it is very convenient to deform an original germ to more general one. For example sometimes this procedure increases the number of singular points and it can be used to derive a contradiction.

6.1. Definition. Let $X$ be a threefold with at worst terminal singularities. We say $X$ is ordinary at $P$ (or $P$ is an ordinary point) if $(X, P)$ is either a cyclic quotient singularity or an ordinary double point.

First, we note that for extremal curve germs deformations are unobstructed: 
6.2. Proposition (Mor88, 1b.8.2], [KM92, 11.4.2], [MP08a, 6.1]). Let $f:(X, C) \rightarrow(Z, o)$ be an extremal curve germ and let $P \in C$. Then every deformation of germs $(X, P) \supset(C, P)$ can be extended to a deformation of $(X, C)$ so that the deformation is trivial outside some small neighborhood of $P$.

Proof. Let $P_{i} \in X$ be singular points. Consider the natural morphism

$$
\Psi: \operatorname{Def}(X) \longrightarrow \prod \operatorname{Def}\left(X, P_{i}\right) .
$$

It is sufficient to show that $\Psi$ is smooth (in particular, surjective). The obstruction to globalizing a deformation in $\prod \operatorname{Def}\left(X, P_{i}\right)$ lies in $R^{2} f_{*} T_{X}$. Since $f$ has only one-dimensional fibers, $R^{2} f_{*} T_{X}=0$. Alternate, more explicit proof can be found in [Mor88, 1b.8.2].

The following theorem was proved in [MP11, Th. 3.2] for $f$ divisorial; in [KM92, (11.4)] for $f$ flipping; or in [MP08a, (6.2)] for $f$ a $\mathbb{Q}$-conic bundle.

6.3. Theorem ([MP08a, (6.2)]). Let $f:(X, C) \rightarrow(Z, o)$ be a divisorial (resp. flipping, $\mathbb{Q}$-conic bundle) curve germ, where $C$ is not necessarily irreducible. Let $\pi: \mathfrak{X} \rightarrow\left(\mathbb{C}_{\lambda}^{1}, 0\right)$ be a flat deformation of $X=\mathfrak{X}_{0}:=$ $\pi^{-1}(0)$ over a germ $\left(\mathbb{C}_{\lambda}^{1}, 0\right)$ with a flat closed subspace $\mathfrak{C} \subset \mathfrak{X}$ such that $C=\mathfrak{C}_{0}$. Then there exists a flat deformation $\mathfrak{Z} \rightarrow\left(\mathbb{C}_{\lambda}^{1}, 0\right)$ and a proper $\mathbb{C}_{\lambda}^{1}$-morphism $\mathfrak{f}: \mathfrak{X} \rightarrow \mathfrak{Z}$ such that $f=\mathfrak{f}_{0}$ and

$$
\mathfrak{f}_{\lambda}:\left(\mathfrak{X}_{\lambda}, \mathfrak{f}_{\lambda}^{-1}\left(o_{\lambda}\right)_{\text {red }}\right) \rightarrow\left(\mathfrak{Z}_{\lambda}, o_{\lambda}\right)
$$

is a divisorial (resp. flipping, $\mathbb{Q}$-conic bundle) extremal curve germ for every small $\lambda$, where $o_{\lambda}:=\mathfrak{f}_{\lambda}\left(\mathfrak{C}_{\lambda}\right)$.

Note however that the deformations do not preserve irreducibility of the central fiber: one can easily construct an example of an extremal curve germ $\left(X, C \simeq \mathbb{P}^{1}\right)$ whose deformation $\left(\mathfrak{X}_{\lambda}, \mathfrak{f}_{\lambda}^{-1}\left(o_{\lambda}\right)_{\text {red }}\right)$ has reducible central fiber. In practice, we often pick up a suitable irreducible component of $\mathfrak{f}_{\lambda}^{-1}\left(o_{\lambda}\right)_{\text {red }}$ and obtain an extremal curve germ whose central fiber is irreducible (see Remark 2.4.2).

6.3.1. Let $f:(X, C) \rightarrow(Z, o)$ be an extremal curve germ with a singular point $P \in C$ of of index $m$, and let $P_{1}, \ldots, P_{r}$ be all the other singular points of $X$ on $C$. Let $\left(X^{\sharp}, P^{\sharp}\right) \rightarrow(X, P)$ be the index one cover and let $\left(X^{\sharp}, P^{\sharp}\right) \subset\left(\mathbb{C}_{x_{1}, \ldots, x_{4}}^{4}, 0\right)$ be an equivariant embedding as in 2.1.1. Let $\phi=0$ be an equation of $X^{\sharp}$. We will choose semi-invariant $\psi \in$ $\mathbb{C}\left\{x_{1}, \ldots, x_{4}\right\}$ with $\operatorname{wt}(\psi) \equiv \operatorname{wt}(\phi) \bmod m$ such that

$$
X_{\lambda, \epsilon}:=\left\{\left(x_{1}, \ldots, x_{4}\right)|\phi+\lambda \psi=0,| x_{i} \mid<\epsilon\right\} / \boldsymbol{\mu}_{m} \subset \mathbb{C}^{4} / \boldsymbol{\mu}_{m}
$$

has only terminal singularities for $|\lambda| \ll \epsilon \ll 1$. 
6.3.2. Proposition (Mor88, 4.7]). For suitable choice of $\psi$, each nearby extremal curve germ $X_{\lambda}^{o} \supset C_{\lambda} \simeq \mathbb{P}^{1}$ contains $P, P_{1}, \ldots P_{r}$ so that $\left(X_{\lambda}^{o}, P_{i}\right) \supset\left(C_{\lambda}, P_{i}\right)$ is naturally isomorphic to $\left(X, P_{i}\right) \supset\left(C, P_{i}\right)$ for all $i$ and $X_{\lambda, \epsilon} \supset C_{\lambda, \epsilon}$ contains all the singularities $\left(\in C_{\lambda}\right)$ of $X_{\lambda} \supset C_{\lambda}$ other than $P_{1}, \ldots P_{r}$. All the singularities of $X_{\lambda, \epsilon} \supset C_{\lambda, \epsilon}$ are ordinary.

If $P$ is a primitive (resp. an imprimitive) point, then $X_{\lambda, \epsilon} \supset C_{\lambda, \epsilon}$ is locally primitive (resp. $P$ is an imprimitive point of $X_{\lambda, \epsilon} \supset C_{\lambda, \epsilon}$ with the same subindex and splitting degree as $X \supset C \ni P)$. Depending on the type of $X \supset C \ni P$, one has

\begin{tabular}{l|l|l|l|l}
\multirow{2}{*}{ type } & \multicolumn{4}{|c}{$X_{\lambda, \epsilon} \supset C_{\lambda, \epsilon}$} \\
\cline { 2 - 4 } & & type & index & $w_{P}(0), w_{P_{i}}(0)$ on $X_{\lambda}$ \\
\hline (IA) & $P$ & (IA) & $m$ & the same as for $X$ \\
(IA $)$ & $P$ & (IA $^{\vee}$ & $m$ & the same as for $X$ \\
(IIA) & $P$ & (IA) & $m$ & the same as for $X$ \\
(II $)$ & $P$ & (IA $)$ & $m$ & the same as for $X$ \\
(IB) & $a_{1}$ points & (IA) & $m$ & the same as for $X$ \\
(IIB) & $P$ and $Q$ & (IA) & 4 and 2 & \\
(III) & $i_{P}(1)$ points & (III) & 1 & 0
\end{tabular}

In the case (III), one can also make $X_{\lambda, \epsilon}$ smooth by choosing some other suitable $\psi$.

6.3.3. Corollary. Arbitrary extremal curve germ $(X, C)$ can be deformed to an extremal curve germ $\left(X^{o}, C^{o}\right)$ with only ordinary points.

6.3.4. Corollary. A flipping extremal curve germ $(X, C)$ has at least one non-Gorenstein point.

Proof. Assume that $(X, C)$ has only type (III) singular points. Applying smoothings as in 6.3.2 repeatedly at type (III) points, one obtains a flipping extremal curve germ $\left(X^{o}, C^{o}\right)$ such that $X^{o}$ is smooth. Thus by (3.1.3) and 4.7 .8 for the normal bundle of $C^{o}$ one has

$$
\operatorname{deg} \mathscr{N}_{C^{o} / X^{o}}=-\operatorname{deg} \operatorname{gr}_{C^{o}}^{1} \mathscr{O}=-1 .
$$

Hence the space of deformation of $C^{o}$ in $X^{o}$ has dimension $\geq 1$. This means that $C^{o}$ moves inside $X^{o}$. This contradicts our assumption that $(X, C)$ is flipping.

If $f: X \rightarrow Z$ is a $K$-negative extremal divisorial contraction from a variety $X$ with terminal $\mathbb{Q}$-factorial singularities, then the target variety $Z$ is also terminal. This is no longer true for divisorial extremal curve germs. The problem is that the exceptional locus of $f$ is not necessarily a divisor in this case (because the $\mathbb{Q}$-factoriality is not assumed). Nevertheless we have the following.

6.4. Theorem ([MP11, Th. 3.1]). Let $f:(X, C) \rightarrow(Z, o)$ be a threedimensional divisorial extremal curve germ, where $C$ is not necessarily 
irreducible, and let $E$ be its exceptional locus. Then the divisorial part of $E$ is a $\mathbb{Q}$-Cartier divisor. If furthermore $C$ is irreducible, then $E$ is $\mathbb{Q}$-Cartier and $(Z, o)$ is a terminal singularity.

6.5. Corollary. Let $f:\left(X, C \simeq \mathbb{P}^{1}\right) \rightarrow(Z, o)$ be a three-dimensional birational extremal curve germ. Then $f$ is divisorial if and only if $(Z, o)$ is a terminal singularity.

The proof uses deformation techniques.

6.5.1. Corollary ([Mor88, Th. 6.3], [MP08a, Prop. 8.3]). An extremal curve germ $(X, C)$ cannot have a point of type (IB).

Proof. Assume that $(X, C)$ has a type (IB) point $P$. We may apply deformations to $(X, C)$ and obtain an extremal curve germ $\left(X^{o}, C^{o} \simeq\right.$ $\left.\mathbb{P}^{1}\right)$ with only ordinary singular points which has at least two points $P^{o}$ and $Q^{o}$ of type (IA) with the same index $m(>1)$. By Proposition $4.8 P^{o}$ and $Q^{\circ}$ are the only non-Gorenstein points of $X^{\circ}$, and $P$ is the only non-Gorenstein point of $X$. Thus by (4.4.1) we have $\mathrm{Cl}^{\mathrm{sc}}\left(X^{o}\right) \simeq$ $\mathbb{Z} \oplus \mathbb{Z} / m \mathbb{Z}$ and so there exists an étale outside $\left\{P^{o}, Q^{o}\right\}$ cyclic cover $\left(X^{\prime}, C^{\prime}\right) \rightarrow\left(X^{o}, C^{o}\right)$ of degree $m$ such that $\left(X^{\prime}, C^{\prime}\right)$ is again an extremal curve germ with $C^{\prime} \simeq \mathbb{P}^{1}$. By Corollary 6.3 .4 the germ $\left(X^{\prime}, C^{\prime}\right)$ and $\left(X^{o}, C^{o}\right)$ cannot be flipping.

Assume that $\left(X^{o}, C^{o}\right)$ is divisorial and let $f^{o}:\left(X^{o}, C^{o}\right) \rightarrow\left(Z^{o}, o^{o}\right)$ be the corresponding contraction. By Theorem 6.4 the point $\left(Z^{\circ}, o^{\circ}\right)$ is terminal and the construction (4.5) shows that $\left(Z^{\circ}, o^{o}\right)$ is of index $m$. According to Kaw92 the exists an exceptional divisor, say $E$, with center $o^{o}$ whose discrepancy equals $a\left(E, Z^{o}\right)=1 / m$. On the other hand, since $E$ is not $f^{o}$-exceptional and the contraction $f^{o}$ is $K$-negative, we have $a\left(E, Z^{\circ}\right)>a\left(E, Z^{\circ}\right)=1 / m$, a contradiction.

Finally, assume that $\left(X^{o}, C^{o}\right)$ is a $\mathbb{Q}$-conic bundle. Since $(X, C)$ has exactly one non-Gorenstein point which is locally primitive, the base $(S, o)$ is smooth. Since $\left(X^{o}, C^{o}\right)$ has two points of the same index $>1$, the base $\left(Z^{o}, o^{o}\right)$ is singular. By 6.3 there exists a deformation family whose general fiber is $\left(Z^{o}, o^{o}\right)$ and the special fiber is $(S, o)$ (in this case a general fiber $\mathfrak{f}_{\lambda}^{-1}\left(o_{\lambda}\right)_{\text {red }}$ must be irreducible). This is impossible.

6.6. Deformation arguments are also used to show the existence of extremal curve germs. Suppose we are given a normal surface germ $(H, C)$ along a curve $C \simeq \mathbb{P}^{1}$ and a contraction $f_{H}: H \rightarrow H_{Z}$ such that $C$ is a fiber. Let $P_{1}, \ldots, P_{r} \in H$ be singular points. Assume also that near each point $P_{i}$ there exists a small one-parameter deformation $H_{t}^{i}$ of $H \cap U_{P_{i}}$, where $U_{P_{i}}$ is a neighborhood of $P_{i}$, such that the total space $V^{i}=\cup H_{t}^{i}$ has terminal singularity at $P_{i}$. Further, by the arguments similar to that in Proposition 6.2 we see that the natural morphism $\operatorname{Def} H \rightarrow \prod \operatorname{Def}\left(H, P_{i}\right)$ is smooth. Hence there exists a global oneparameter deformation $H_{t}$ of $H$ which induces a local deformation of 
$H_{t}^{i}$ near each $P_{i}$. Then we construct a threefold $X$ as a total oneparameter deformation space $X=\cup H_{t}$. This shows the existence of $X \supset C$ with $H \in\left|\mathscr{O}_{X}\right|$ and such that $P_{i} \in C \cap U_{P_{i}} \subset U_{P_{i}}$ has the desired structure. Note however that $H$ may not be general in $\left|\mathscr{O}_{X}\right|$.) The contraction $f: X \rightarrow Z$ exists by arguments similar to KM92, 11.4.1] and Theorem 6.3. The contraction is birational (resp. $\mathbb{Q}$-conic bundle) if $H_{Z}$ is a surface (resp. a curve).

\section{General member of $\left|-K_{X}\right|$}

7.1. Let $X$ be a threefold having terminal singularities only and let $D$ be an effective integral $\mathbb{Q}$-Cartier divisor on $X$. Then $D$ is CohenMacaulay [KM98, Cor. 5.25]. Therefore, we have

- if $D$ has only isolated singularities, then $D$ is normal;

- if $D \sim-K_{X}$, then $D$ is Gorenstein.

In certain situations we can say more:

7.2. Theorem ([Rei87, $(6.4 \mathrm{~B})])$. Let $(X, P)$ be a three-dimensional terminal singularity. Then a general member of $\left|-K_{X}\right|$ has at most a Du Val singularity at $P$.

7.2.1. Depending on the types of terminal singularities, a general member $D \in\left|-K_{X}\right|$ and its preimage $D^{\sharp}$ under the index-one cover are described below (see [Rei87, (6.4B)]).

\begin{tabular}{c|c|c|c|c} 
name & equation of $D^{\sharp}$ & $\boldsymbol{\mu}_{m^{-}}$-action & cover $D^{\sharp} \rightarrow D$ & $\mathrm{aw}(X, P)$ \\
\hline $\mathrm{cA} / \mathrm{m}$ & $x y+z^{k}$ & $(1,-1,0)$ & $\mathrm{A}_{\mathrm{k}-1} \stackrel{m: 1}{\longrightarrow} \mathrm{A}_{\mathrm{km}-1}$ & $k$ \\
$\mathrm{cAx} / 4$ & $x^{2}+y^{2}+z^{2 k-1}$ & $(1,3,2)$ & $\mathrm{A}_{2 \mathrm{k}-2} \stackrel{4: 1}{\longrightarrow} \mathrm{D}_{2 \mathrm{k}+1}$ & $2 k-1$ \\
$\mathrm{cD} / 3$ & $x^{2}+y^{3}+z^{3}$ & $(0,1,2)$ & $\mathrm{D}_{4} \stackrel{3: 1}{\longrightarrow} \mathrm{E}_{6}$ & 2 \\
$\mathrm{cAx} / 2$ & $x^{2}+y^{2}+z^{2 k}$ & $(0,1,1)$ & $\mathrm{A}_{2 \mathrm{k}-1} \stackrel{2: 1}{\longrightarrow} \mathrm{D}_{\mathrm{k}+2}$ & 2 \\
$\mathrm{cD} / 2$ & $x^{2}+y^{2} z+z^{k}$ & $(1,1,0)$ & $\mathrm{D}_{\mathrm{k}+1} \stackrel{2: 1}{\longrightarrow} \mathrm{D}_{2 \mathrm{k}}$ & $k$ \\
$\mathrm{cE} / 2$ & $x^{2}+y^{3}+z^{4}$ & $(1,0,1)$ & $\mathrm{E}_{6} \stackrel{2: 1}{\longrightarrow} \mathrm{E}_{7}$ & 3
\end{tabular}

M. Reid conjectured that an analog of 7.2 holds for any $K$-negative contraction of terminal threefolds (general elephant). The conjecture is very important in birational geometry. The following theorem shows that this conjecture is true for extremal curve germs. Different parts of this theorem were proved in [Mor88, KM92] [MP08a], [MP09].

7.3. Theorem. Let $\left(X, C \simeq \mathbb{P}^{1}\right)$ be an extremal curve germ. Then a general member of the linear system $\left|-K_{X}\right|$ is normal and has only Du Val singularities.

Note that by the inversion of adjunction [Sho93, § 3], [Kol92, Ch. 17] the Du Val property of a general member $D \in\left|-K_{X}\right|$ is equivalent to the plt property of the pair $(X, D)$. 
All the possibilities for general members of $\left|-K_{X}\right|$ have been classified, see [KM92] and [MP09]. Below we reproduce this classification in the case where $\left(X, C \simeq \mathbb{P}^{1}\right)$ has only one non-Gorenstein point.

7.4. Theorem. Let $f:\left(X, C \simeq \mathbb{P}^{1}\right) \rightarrow(Z, o)$ be an extremal curve germ. Assume that $(X, C)$ has only one non-Gorenstein point $P$. Let $D \in\left|-K_{X}\right|$ be a general member. If $f$ is birational, we let $D_{Z}:=f(D)$ which is a general member of $\left|-K_{Z}\right|$. If $f$ is a $\mathbb{Q}$-conic bundle, we let $D_{Z}:=\operatorname{Spec}_{Z} f_{*} \mathscr{O}_{D}$. Then $D$ and $D_{Z}$ have only Du Val singularities and the morphism $f_{D}: D \rightarrow D_{Z}$ is birational and crepant. Moreover, only one of the following possibilities holds.

7.4.1 ([KM92, (2.2.1), (2.2.1')], [MP08a, (1.2.3)-(1.2.6)]). We have $D \cap$ $C=\{P\}$ and $f_{D}: D \rightarrow D_{Z}$ is an isomorphism. In this case, $D$ induces a general member of $\left|-K_{(X, P)}\right|$, and $\Delta(D)$ is described by 7.2 .1 .

7.4.2 ([KM92, (2.2.2)], [MP09, 1.3.1]). $P \in(X, C)$ is of type (IC),

$$
\Delta(D, C): \underbrace{o-\cdots-\circ}_{m-3}-\stackrel{\circ}{\mathrm{l}}-\diamond,
$$

where $m$, the index of $(X, P)$, is odd and $m \geq 5$.

7.4.3 ([KM92, (2.2.2')], [MP09, 1.3.2]). $P \in(X, C)$ is of type (IIB),

$$
\Delta(D, C): \quad \circ-\circ-\stackrel{\circ}{\mathrm{l}} \mathrm{\circ} \odot-\diamond .
$$

In some cases of 7.4 .1 there are additional restrictions on the general member $D \in\left|-K_{X}\right|$. For example, in the case where $f$ is birational and $(X, P)$ is of type cAx/2, the general $D \in\left|-K_{X}\right|$ is of type $\mathrm{D}_{4}$ [KM92, 4.8.5.7]. A lot of restrictions are imposed on imprimitive $\mathbb{Q}$ conic bundles (see 9.1).

7.5. Let us outline the main ideas of the proof in the case where $(X, C)$ has only one non-Gorenstein point. Thus, let $(X, C)$ be an extremal curve germ with a unique non-Gorenstein point $P$.

7.5.1. Lemma (see Mor88, Theorem 7.3], [MP08a, § 7, 8.6.1]). In the notation of 7.4 and with the symbols in Propositions 5.4 for primitive points and 5.5 for imprimitive points, we have.

(i) If $P \in(X, C)$ is of type (IA), (IIA), (IA $\left.{ }^{\vee}\right),\left(\mathrm{IIA}^{\vee}\right),\left(\mathrm{ID}^{\vee}\right)$, or $\left(\mathrm{IE}^{\vee}\right)$, then for a general member $D \in\left|-K_{X}\right|$ we have $D \cap C=\{P\}$.

(ii) If $P \in(X, C)$ is of type (IC) or (IIB), then for a general member $S \in\left|-2 K_{X}\right|$ we have $S \cap C=\{P\}$. Moreover, the pair $\left(X, \frac{1}{2} S\right)$ is klt. 
Sketch of the proof. Consider the case where $P$ is of type (IA). Take $\psi:=x_{2}+\psi_{\bullet}$, where $\psi_{\bullet} \in \mathbb{C}\left\{x_{1}, \ldots, x_{4}\right\}$ is a sufficiently general semiinvariant with $\operatorname{wt}\left(\psi_{\bullet}\right) \equiv \operatorname{wt}\left(x_{2}\right)$ and let $D:=\{\psi=0\} / \boldsymbol{\mu}_{m}$. Then $D \cap C=\{P\}$ and $\operatorname{wt}(\psi) \equiv \operatorname{wt}(\Omega)$, where $\Omega=\operatorname{Res}\left(\phi^{-1} d x_{1} \wedge \cdots \wedge d x_{4}\right)$. Therefore, $\psi \Omega^{-1} \in \mathscr{O}_{X}\left(-K_{X}\right)$ in a neighborhood of $P$. Since $P$ is the only non-Gorenstein point, this implies that $K_{X}+D$ is Cartier globally by (4.4.1). On the other hand, $D \cdot C=\frac{1}{m}$ ord $\psi=\frac{a_{2}}{m}<1$ and $-K_{X} \cdot C<1$ (see (3.1.3) and 5.5.1). Therefore, $K_{X}+D \equiv 0$. Then Corollary 2.4.1](iii) implies $K_{X}+D \sim 0$. Other cases are treated similarly.

Thus in the cases of 7.5.1) we are done. Cases (IC) and (IIB) are much more delicate. Rough idea of proof in these cases is to use surjectivity of the restriction map

$$
H^{0}\left(X, \mathscr{O}_{X}\left(-K_{X}\right)\right) \longrightarrow H^{0}\left(S, \mathscr{O}_{S}\left(-K_{X}\right)\right)
$$

and extend a "good" member of $\left.\left|-K_{X}\right|\right|_{S}$ to $X$.

7.6. Kawamata Kaw88] had shown that Theorem 7.3 in the flipping case is a sufficient condition for the existence of flips. Indeed, applying a Bertini type arguments (see [KM98, Corollary 2.33]) one can show that, for a general member $S \in\left|-2 K_{X}\right|$, the pair $\left(X, \frac{1}{2} S\right)$ is klt. Consider the double cover $\left(X^{b}, C^{b}\right) \rightarrow(X, C)$ branched over $S$. Then $X^{b}$ has only canonical singularities (see [KM98, 5.20], [Mor88, 7.2]) and admits a flopping contraction of $C^{b}$. Then the existence of flip for $(X, C)$ follows from the existence of flop for $\left(X^{b}, C^{b}\right)$.

7.7. As a corollary of Theorem 7.3 we have the following fact which was conjectured by V. Iskovskikh [Isk96].

7.7.1. Corollary ([Pro97], MP08a] $)$. Let $f:(X, C) \rightarrow(Z, o)$ be a $\mathbb{Q}$ conic bundle germ. Then $(Z, o)$ is either smooth or a Du Val singularity of type A.

The corollary has important applications in birational geometry of conic bundles (see [Isk96], [Pro18]).

\section{INDEX TWO GERMS}

In this section we discuss extremal curve germs having index two points only. The methods are different from those used in other sections. Throughout this section we do not assume that the central curve of an extremal curve germ is irreducible.

8.1. Proposition ([KM92, 4.6]). Let $(X, C)$ be an extremal curve germ of index two. If $(X, C)$ is a $\mathbb{Q}$-conic bundle germ, then we assume that the base surface is smooth. Then we have the following. 
(i) If $P$ is a point of index two, then $P$ is the only non-Gorenstein point, all the components of $C$ pass through $P$ and they do not meet each other elsewhere.

(ii) Each germ $\left(X, C_{i}\right)$ is of type (IA) at $P$.

(iii) A general member $F \in\left|-K_{X}\right|$ satisfies $F \cap C=\{P\}$ and has only Du Val singularity at $P$.

Proof. By Lemma 3.7.1 it is sufficient to show that every irreducible component of $C$ has at most one non-Gorenstein point. Assume the converse: a component $C_{i} \subset C$ contains two points $P$ and $Q$ of index two. If $\operatorname{gr}_{C_{i}}^{0} \not \mathscr{O}_{C}(-1)$, then $\left(X, C_{i}\right)$ is a $\mathbb{Q}$-conic bundle germ by Corollary 3.7](i), In this case, $C=C_{i}$ and $\left(X, C_{i}\right)$ is primitive (because the base is smooth). This contradicts Corollary 4.7.8. Thus $\operatorname{gr}_{C_{i}}^{0} \simeq$ $\mathscr{O}_{C}(-1)$. Since the numbers $K_{X} \cdot C_{i}, w_{P}(0)$, and $w_{Q}(0)$ are strictly positive and contained in $\frac{1}{2} \mathbb{Z}$, we get a contradiction by (3.1.2).

(ii) follows from 5.4 (the case (IB) is excluded by Corollary 6.5.1). (iii) is proved as in Sect. 7

First, we consider the birational case following [KM92, §4].

8.2. Theorem ([KM92, 4.7]). Let $(X, C)$ be a birational extremal curve germ of index two. Let $P \in X$ be a non-Gorenstein point. Then a general member $H \in\left|\mathscr{O}_{X}\right|$ is normal and has only rational singularities. The following are the only possibilities for the dual graph $\Delta(H, C)$, where $\stackrel{3}{\circ}-\underbrace{\mathrm{a} \ldots \ldots-\cdots}_{n-2}-\stackrel{3}{\circ}$ should be replaced with $\stackrel{4}{\circ}$ if $n=1$.

The following is the only flipping case.

8.2.1. Then $C \simeq \mathbb{P}^{1}$, the singularity $(X, P)$ is of type $\mathrm{cA} / 2,\left(H_{Z}, o\right)$ is of type $\frac{1}{2 n+1}(1,2 n-1)$, and

$$
\Delta(H, C): \quad \bullet-\stackrel{3}{\circ}-\underbrace{\mathrm{o}-\cdots-\mathrm{o}}_{n-2}-\stackrel{3}{\mathrm{o}}
$$

In the remaining cases $(X, C)$ is divisorial. Then $(Z, o)$ is a $\mathrm{cDV}$ point and $\left(H_{Z}, o\right)$ is a Du Val singularity. If we say that $\left(H_{Z}, o\right)$ is of type $\mathrm{A}_{0}$, this means that it is smooth.

\begin{tabular}{|c|c|c|c|}
\hline No. & $(X, P)$ & $\left(H_{Z}, o\right)$ & $\Delta(H, C)$ \\
\hline \multicolumn{4}{|c|}{$C$ has one component } \\
\hline 8.2 .2 & $\mathrm{cA} / 2$ & $\mathrm{~A}_{1}$ & $\mathrm{o}-\bullet-\mathrm{o}^{3}-\underbrace{\mathrm{o}-\cdots-\mathrm{o}}-\mathrm{o}^{3}$ \\
\hline 8.2 .3 & $\mathrm{cA} / 2$ & $\mathrm{~A}_{0}$ & o- $\mathrm{O}-\bullet-\stackrel{4}{\circ}$ \\
\hline 8.2 .4 & $\mathrm{cA} / 2$ & $\mathrm{~A}_{2}$ & $\stackrel{3}{\circ}-\circ-{ }^{3}$ \\
\hline
\end{tabular}




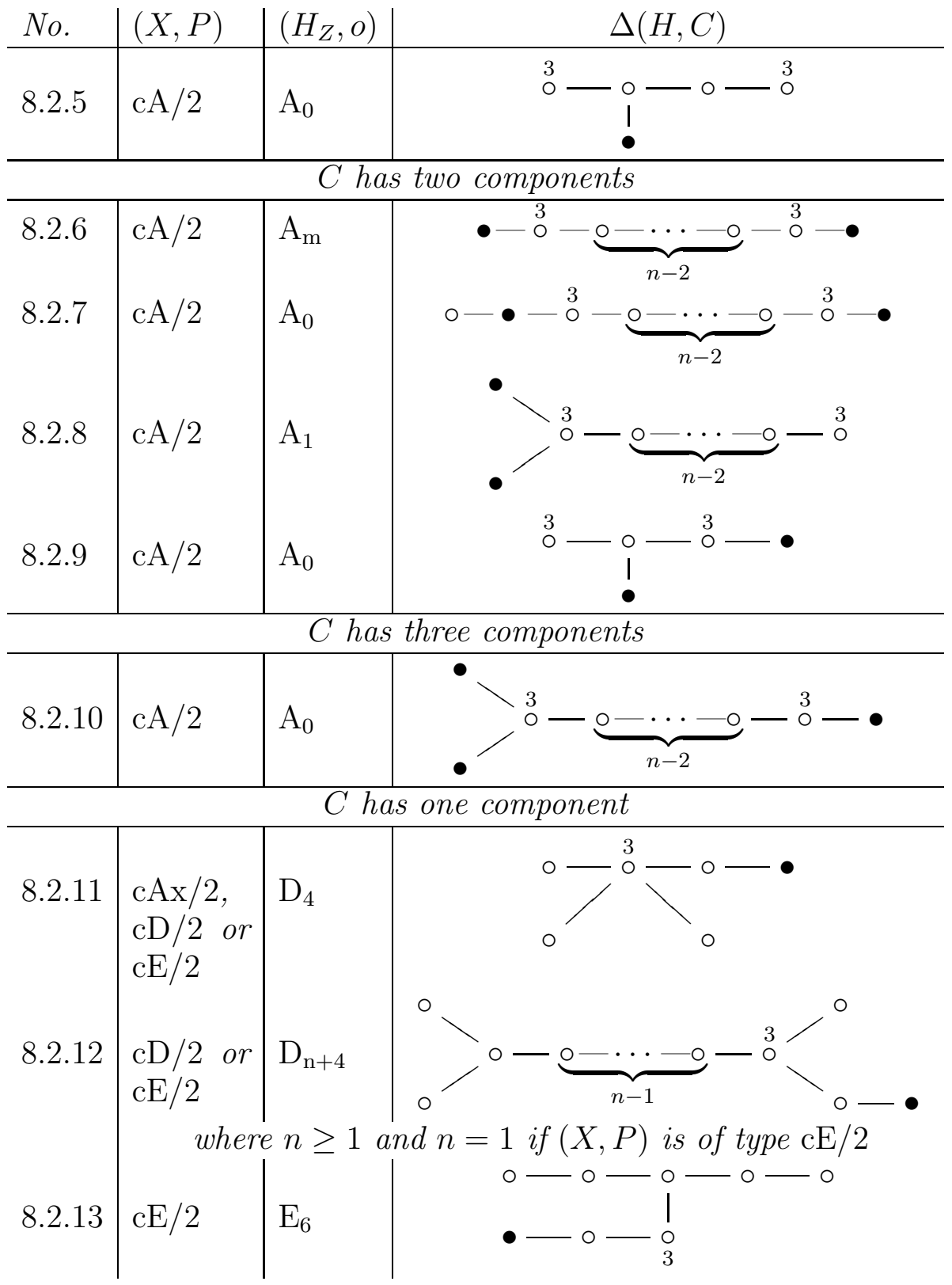

Note that the singularities of $H$ are log terminal in all the cases except for 8.2.11, 8.2.12, 8.2.13. In the cases 8.2.11 and 8.2.12 the singularities of $H$ are $\log$ canonical.

8.2.14. Theorem ([KM92, 4.2]). In the notation of Theorem 8.2 assume that $f$ is flipping (see 8.2.1) and let $(X, C) \rightarrow\left(X^{+}, C^{+}\right)$be the corresponding flip. Then the following hold.

(i) In appropriate coordinates the point $(X \ni P)$ is given by

$$
\left\{x_{1} x_{2}+p\left(x_{3}^{2}, x_{4}\right)=0\right\} / \boldsymbol{\mu}_{2}(1,1,1,0)
$$


and $C$ is the $x_{1}$-axis.

(ii) $X^{+}$has at most one singular point which is isolated $\mathrm{cDV}$ with equation $x_{1} x_{2}+p\left(x_{3}, x_{4}\right)=0$ and $C^{+}$is the $x_{1}$-axis.

(iii) $(Z, o)$ is a rational triple point given by the $2 \times 2$-minors of the matrix

$$
\left(\begin{array}{ccc}
z_{1} & z_{2} & z_{3} \\
z_{2} & z_{5} & p\left(z_{1}, z_{4}\right)
\end{array}\right)
$$

The proofs use the following standard construction.

8.2.15. Construction. Let $C_{i} \subset C$ be the irreducible components of $C$. Since $X$ has only points of index one and two, $m_{i}=-2 K_{X} \cdot C_{i}$ is a positive integer. Let $E_{i} \subset X$ be the union of $m_{i}$ disjoint discs transversal to $C_{i}$ and let $E=\sum E_{i}$. Then $E \in\left|-2 K_{X}\right|$. Hence we can take the corresponding double cover $X^{\prime} \rightarrow X$ branched over $E$. Here $X^{\prime}$ has only index one terminal singularities. Let $E^{\prime} \subset X^{\prime}$ be the preimage of $E$. The natural map $E^{\prime} \rightarrow E$ is an isomorphism. The Stein factorization induces the following diagram

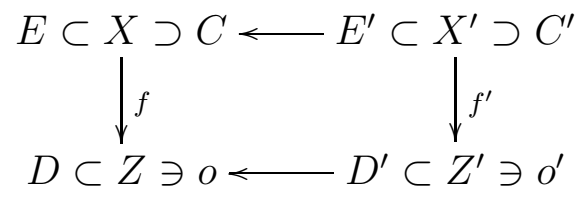

where $D:=f(E)$ and $D^{\prime}:=f\left(E^{\prime}\right)$. Here $Z^{\prime} \rightarrow Z$ is a double cover branched over $D$. By construction, $f^{\prime}$ is crepant with respect to $K_{X^{\prime}}$ and the fibers of $f^{\prime}$ have dimension $\leq 1$. Therefore, $Z^{\prime}$ has $\mathrm{cDV}$ points only (if $f$ is divisorial, then $Z^{\prime}$ has a double curve).

Sketch of the proof of 8.2 and 8.2 .14 . The above construction defines a $\boldsymbol{\mu}_{2}$-action on $X^{\prime} / Z^{\prime}$ and the quotient is $X / Z$. The fixed point set of the action on $Z^{\prime}$ is precisely $D^{\prime}$. Since $Z^{\prime} \ni o^{\prime}$ is a cDV point, it is a hypersurface in $\mathbb{C}^{4}$, thus it can be written down explicitly. This will enable us to get equations for $X$ and $Z$. We have an $\boldsymbol{\mu}_{2}$-equivariant embedding $\left(Z^{\prime}, o^{\prime}\right) \subset\left(\mathbb{C}_{y_{1}, \ldots, y_{4}}^{4}, 0\right)$, and we may assume that the coordinates are eigenvectors and $y_{1}, \ldots, y_{j}$ are those of weights 1 . Thus $D^{\prime}=\left\{y_{1}=\cdots=y_{j}=0\right\} \cap Z^{\prime}$. Hence $j=1$ or 2 .

8.2.16. Claim. (i) If $D^{\prime}$ is Cartier, then $f$ is divisorial and $D$ is singular along $f(E)$, where $E$ is the $f$-exceptional divisor.

(ii) If $D^{\prime}$ is not Cartier, then $f$ is flipping, $D$ is smooth and $C$ is irreducible.

Proof. If $j=1$ then $D^{\prime}$ is Cartier. In this case, $f$ must be divisorial. Indeed, otherwise since $f^{\prime}$ is an isomorphism outside the origin and $E^{\prime}$ is $f^{\prime}$-ample, $D^{\prime}$ cannot be Cartier. Hence $f$ contracts an exceptional divisor $E \subset X$. Then for a general fiber $l$ of $E$ we have $K_{X} \cdot l=-1$. Hence $E \cdot F=2$. Therefore, $D$ has a double curve along the image of 
$E$ and is smooth elsewhere. If $E$ is chosen generically, then $D$ has an ordinary double curve along the image of $E$.

Assume that $j=2$. Then $\left\{y_{1}=y_{2}=0\right\}$ must be contained in $Z^{\prime}$. Furthermore, $D^{\prime}$ is irreducible and this implies that $C$ is irreducible. Then $E \rightarrow D$ is an isomorphism outside the origin, in fact, it turns out to be an isomorphism. In particular, $D$ is smooth. This implies that $f$ is flipping.

First consider the flipping case. Since $\left\{y_{1}=y_{2}=0\right\} \subset Z^{\prime}$, the equation of $Z^{\prime}$ can be written in the form $y_{1} \phi_{1}+y_{2} \phi_{2}=0$. If $\operatorname{wt}\left(\phi_{1}\right)=$ $\operatorname{wt}\left(\phi_{2}\right)=1$, then $y_{1} \phi_{1}+y_{2} \phi_{2} \in\left(y_{1}, y_{2}\right)^{2}$, which implies that $Z^{\prime}$ is singular along $\left\{y_{1}=y_{2}=0\right\}$. This is impossible. Thus $\operatorname{wt}\left(\phi_{1}\right)=\operatorname{wt}\left(\phi_{2}\right)=1$. Since $Z^{\prime}$ is a double point, either $\phi_{1}$ or $\phi_{2}$ must contain a linear term. Assume that $\phi_{1}$ contains $y_{j}$. By wt reasons $j=3$ or 4 . Now we can rewrite the equation in the following form

$$
y_{1} y_{3}+y_{2} p\left(y_{2}^{2}, y_{4}\right)=0 \text {. }
$$

With this explicit equation we can easily compute everything. The variety $X$ is obtained by blowing up $\left\{y_{2}=y_{3}=0\right\}$ and taking quotient by the group action. This gives us one singular point with the required equation. The flipped variety $X^{+}$is obtained by blowing up $\left\{y_{1}=y_{2}=\right.$ $0\}$ and taking quotient by the group action. To get equations for $(Z, o)$, we note that the invariants of the $\boldsymbol{\mu}_{2}$-action on $\mathbb{C}\left\{y_{1}, \ldots, y_{4}\right\}$ are

$$
z_{1}=y_{2}^{2}, \quad z_{2}=y_{1} y_{2}, \quad z_{3}=y_{3}, \quad z_{4}=y_{4}, \quad z_{5}=y_{1}^{2} .
$$

We get exactly the equations given by the minors of the matrix in the assertion of the theorem. A hyperplane section given by $z_{4}=c z_{1}$.

Now consider the divisorial case. Then $D^{\prime} \subset Z^{\prime}$ is Cartier and the $\boldsymbol{\mu}_{2}$-action is given by $\operatorname{wt}(y)=(0,0,0,1)$. Let $D^{\prime}$ be given by $y_{4}=$ $\psi\left(y_{1}, y_{2}, y_{3}\right)=0$. Thus we can write the equation of $Z^{\prime}$ in the form

$$
y_{4}^{2} \phi\left(y_{1}, \ldots, y_{4}\right)+\psi\left(y_{1}, y_{2}, y_{3}\right)=0 \text {. }
$$

Since $f^{\prime}$ is crepant, $Z^{\prime}$ cannot be smooth, in particular, $\operatorname{mult}_{0}(\psi) \geq 2$. The equation of $Z$ is now given by

$$
t \phi\left(y_{1}, y_{2}, y_{3}, t\right)+\psi\left(y_{1}, y_{2}, y_{3}\right)=0, \quad\left(t=y_{4}^{2}\right) .
$$

In particular, this shows that $(Z, o)$ is an (isolated) cDV point (cf. 6.5). Now the proof proceeds by a careful analysis of the equations. See [KM92, § 4] for details.

8.3. Now we consider $\mathbb{Q}$-conic bundles. The case of singular base surface is easy:

8.3.1. Proposition ([Pro97, § 3], [MP08b] $)$. A $\mathbb{Q}$-conic bundle of index two over a singular base is either of type 9.1 .2 or toroidal 2.3 . 
Index two $\mathbb{Q}$-conic bundles over a smooth base were classified in [Pro97, §3] and [MP08a]. Similar to birational case these are quotients of some elliptic fibrations by an involution. On the other hand, one can note that there exists an embedding to a relative weighted projective space:

8.4. Theorem. Let $f:(X, C) \rightarrow(Z, o)$ be a $\mathbb{Q}$-conic bundle germ of index two. Assume that $(Z, o)$ is smooth. Fix an isomorphism $(Z, o) \simeq$ $\left(\mathbb{C}^{2}, 0\right)$. Then there is an embedding

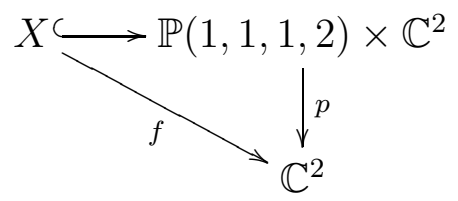

such that $X$ is given by two equations

$$
\begin{aligned}
& q_{1}\left(y_{1}, y_{2}, y_{3}\right)-\psi_{1}\left(y_{1}, \ldots, y_{4} ; u, v\right)=0, \\
& q_{2}\left(y_{1}, y_{2}, y_{3}\right)-\psi_{2}\left(y_{1}, \ldots, y_{4} ; u, v\right)=0,
\end{aligned}
$$

where $\psi_{i}$ and $q_{i}$ are weighted quadratic in $y_{1}, \ldots, y_{4}$ with respect to $\operatorname{wt}\left(y_{1}, \ldots, y_{4}\right)=(1,1,1,2)$ and $\psi_{i}\left(y_{1}, \ldots, y_{4} ; 0,0\right)=0$. The only nonGorenstein point of $X$ is $(0,0,0,1 ; 0,0)$. Up to projective transformations, the following are the possibilities for $q_{1}$ and $q_{2}$ :

\begin{tabular}{l|l|l|l}
\hline no. & $q_{1}$ & $q_{2}$ & $f^{-1}(o)$ \\
\hline 8.4 .3 & $y_{1}^{2}-y_{2}^{2}$ & $y_{1} y_{2}-y_{3}^{2}$ & $C_{1}+C_{2}+C_{3}+C_{4}$ \\
8.4 .4 & $y_{1} y_{2}$ & $\left(y_{1}+y_{2}\right) y_{3}$ & $2 C_{1}+C_{2}+C_{3}$ \\
8.4 .5 & $y_{1} y_{2}-y_{3}^{2}$ & $y_{1} y_{3}$ & $3 C_{1}+C_{2}$ \\
8.4 .6 & $y_{1}^{2}-y_{2}^{2}$ & $y_{3}^{2}$ & $2 C_{1}+2 C_{2}$ \\
8.4 .7 & $y_{1} y_{2}-y_{3}^{2}$ & $y_{1}^{2}$ & $4 C_{1}$ \\
8.4 .8 & $y_{1}^{2}$ & $y_{2}^{2}$ & $4 C_{1}$
\end{tabular}

Conversely, if $X \subset \mathbb{P}(1,1,1,2) \times \mathbb{C}^{2}$ is given by equations of the form (8.4.2) and singularities of $X$ are terminal, then the projection $f:\left(X, f^{-1}(0)_{\text {red }}\right) \rightarrow\left(\mathbb{C}^{2}, 0\right)$ is a $\mathbb{Q}$-conic bundle of index two.

8.4.9. Remark. A general member $H \in\left|\mathscr{O}_{X}\right|$ is normal in the case 8.4.7 and non-normal in the case 8.4.8.

Sketch of the proof. First we prove the last statement. By our assumption $X$ has only terminal singularities. Then $X$ does not contain the surface $\left\{y_{1}=y_{2}=y_{3}=0\right\}=\operatorname{Sing}\left(\mathbb{P} \times \mathbb{C}^{2}\right)$ (otherwise both $\psi_{1}$ and $\psi_{2}$ do not depend on $\left.y_{4}\right)$. By the adjunction formula, $K_{X}=-\left.L\right|_{X}$, where $L$ is a Weil divisor on $\mathbb{P} \times \mathbb{C}^{2}$ such that the restriction $\left.L\right|_{\mathbb{P}}$ is $\mathscr{O}_{\mathbb{P}}(1)$. Therefore, $X \rightarrow \mathbb{C}^{2}$ is a $\mathbb{Q}$-conic bundle. It is easy to see that the only non-Gorenstein point of $X$ is $(0,0,0,1 ; 0,0)$ and it is of index two. 
Now let $f:(X, C) \rightarrow(Z, o) \simeq\left(\mathbb{C}^{2}, 0\right)$ be a $\mathbb{Q}$-conic bundle germ of index two. Let $P \in X$ be a point of index two. Let $\pi:\left(X^{\sharp}, P^{\sharp}\right) \rightarrow$ $(X, P)$ be the index-one cover. We need the following lemma.

8.4.10. Lemma ([MP08a, 12.1.9]). Let $F^{\sharp}=\pi^{-1}(F)_{\text {red }}$ be the pull-back of $F$. Let $\Gamma:=f^{-1}(o)$ be the scheme fiber and let $\Gamma^{\sharp}=\pi^{-1}(\Gamma)$. Then we have

$$
\mathscr{O}_{F^{\sharp} \cap \Gamma^{\sharp}} \simeq \mathbb{C}[x, y] /\left(x y, x^{2}+y^{2}\right) .
$$

Furthermore, the $\boldsymbol{\mu}_{2}$-action is given by wt $(x, y) \equiv(1,1) \bmod 2$.

Using this lemma one can apply arguments of [Mor75, pp. 631-633] to get the desired embedding $X \subset \mathbb{P}(1,1,1,2) \times Z$ considering the graded anti-canonical $\mathscr{O}_{Z}$-algebra

$$
\mathscr{R}:=\bigoplus_{i \geq 0} \mathscr{R}_{i}, \quad \text { where } \quad \mathscr{R}_{i}:=H^{0}\left(\mathscr{O}_{X}\left(-i K_{X}\right)\right) .
$$

We sketch the main idea.

Let $w$ be a local generator of $\mathscr{O}_{X^{\sharp}}\left(-K_{X}\right)$ at $P^{\sharp}$, let $u, v$ be coordinates on $Z=\mathbb{C}^{2}$, and let $z=0$ be the local equation of $F^{\sharp}$ in $\left(X^{\sharp}, P^{\sharp}\right)$. Using the vanishing of $H^{1}\left(\mathscr{O}_{X}\left(-K_{X}\right)\right)$ for $i>0$ and the exact sequence

$$
0 \rightarrow \mathscr{O}_{X}\left(-(i-1) K_{X}\right) \rightarrow \mathscr{O}_{X}\left(-i K_{X}\right) \rightarrow \mathscr{O}_{F}\left(-i K_{X}\right) \rightarrow 0
$$

one can see

$$
\mathscr{R}_{i} /(z w) \mathscr{R}_{i-1} \simeq H^{0}\left(\mathscr{O}_{F}\left(-i K_{X}\right)\right), \quad i>0
$$

Therefore,

$$
\mathscr{R}_{i} /(z w) \mathscr{R}_{i-1}+(u, v) \mathscr{R}_{i}=\left(\mathscr{O}_{F^{\sharp} \cap \Gamma^{\sharp}}\left(-i K_{X}\right)\right)^{\boldsymbol{\mu}_{2}} .
$$

By Lemma 8.4.10 we have an embedding

$$
\mathscr{R} /(z w, u, v) \mathscr{R} \hookrightarrow\left(\mathbb{C}[x, y, w] /\left(x y, x^{2}+y^{2}\right)\right)^{\boldsymbol{\mu}_{2}} .
$$

Using $\mathscr{R}_{0} /(u, v) \mathscr{R}_{0}=\mathbb{C}$, one can easily see that

$$
\mathscr{R} /(z w, u, v) \mathscr{R}=\mathbb{C}\left[y_{1}, y_{2}, y_{4}\right] /\left(y_{1} y_{2}, y_{1}^{2}+y_{2}^{2}\right),
$$

where $y_{1}=x w, y_{2}=y w, y_{4}=w^{2}$. Put $y_{3}:=z w$. Then similar to [Mor75, pp. 631-633] we obtain

$$
\mathscr{R} \simeq \mathscr{O}_{Z}\left[y_{1}, y_{2}, y_{3}, y_{4}\right] / \mathscr{I},
$$

where $\mathscr{I}$ is generated by the following regular sequence

$$
\begin{array}{ll}
y_{1} y_{2}+y_{3} \ell_{1}\left(y_{1}, \ldots, y_{3}\right) & +\psi_{1}\left(y_{1}, \ldots, y_{4} ; u, v\right), \\
y_{1}^{2}+y_{2}^{2}+y_{3} \ell_{2}\left(y_{1}, \ldots, y_{3}\right) & +\psi_{2}\left(y_{1}, \ldots, y_{4} ; u, v\right)
\end{array}
$$

with $\psi_{i}\left(y_{1}, \ldots, y_{4} ; 0,0\right)=0$.

Note also that the construction 8.2.15 in the $\mathbb{Q}$-conic bundle case produces an elliptic fibration. It can be used for classification (see [Pro97, $\S 3])$. 
8.4.11. Example. Let $X \subset \mathbb{P}(1,1,1,2) \times \mathbb{C}_{u, v}^{2}$ is given by the equations

$$
\begin{aligned}
y_{1} y_{2} & =\left(a u+b u^{2}+c u v\right) y_{4}, \\
\left(y_{1}+y_{2}+y_{3}\right) y_{3} & =v y_{4},
\end{aligned}
$$

where $a, b, c \in \mathbb{C}$ are constants. It is easy to check that the projection $X \rightarrow \mathbb{C}^{2}$ is a $\mathbb{Q}$-conic bundle as in 8.4.3. The only singular point is of type $\mathrm{cA} / 2$. If $a \neq 0$, then this point is a cyclic quotient of type $\frac{1}{2}(1,1,1)$.

8.4.12. Example. Let $X \subset \mathbb{P}(1,1,1,2) \times \mathbb{C}_{u, v}^{2}$ is given by the equations

$$
\begin{aligned}
& y_{1}^{2}=u y_{3}^{2}+v y_{4} \\
& y_{2}^{2}=u y_{4}+v y_{3}^{2}
\end{aligned}
$$

Then the projection $X \rightarrow \mathbb{C}^{2}$ is a $\mathbb{Q}$-conic bundle of type 8.4 .8 containing one singular point of type $\frac{1}{2}(1,1,1)$ and two ordinary double points.

More examples are given in [MP11, $\S 7$ and Remark 6.7.1], and [Pro97, $\S 3]$. It can be shown [MP11, §7] that every type of terminal index two singularity can occur on some index two $\mathbb{Q}$-conic bundle as in 8.4 .7 or 8.4.8,

\section{LOCALLY IMPRIMITIVE GERMS}

In this section we collect the results concerning extremal curve germs with a locally imprimitive point. Note that in this case the imprimitive point is unique and the splitting cover is locally primitive along arbitrary irreducible component of the central curve (see Corollary 4.6.4). Moreover, one can show that the imprimitive point is the only nonGorenstein point, see [Mor88, Th. 6.7, 9.4] and [MP08a, § 7].

The following theorem summarizes the results contained in [Mor88, [KM92, MP08a, [MP11.

9.1. Theorem. Let $f:\left(X, C \simeq \mathbb{P}^{1}\right) \rightarrow(Z, o)$ be an extremal curve germ such that $(X, C)$ is locally imprimitive. Let $P \in X$ be the imprimitive point and let $m, s$ and $\bar{m}$ be its index, splitting degree and subindex, respectively. In this case, $P$ is the only non-Gorenstein point and $X$ has at most one type (III) point. Then one of the following holds.

9.1.1 ([MP08a, 1.2.3]). $f$ is a $\mathbb{Q}$-conic bundle, $(X, C)$ is of type $\left(\mathrm{IE}^{\vee}\right)$ at $P,(Z, o)$ is $D u$ Val of type $\mathrm{A}_{3}, X$ has a cyclic quotient singularity $P$ of type $\frac{1}{8}(5,1,3)$ and has no other singular points. Furthermore, $(X, C)$ is the quotient of the index-two $\mathbb{Q}$-conic bundle germ given by the following two equations in $\mathbb{P}(1,1,1,2)_{y_{1}, \ldots, y_{4}} \times \mathbb{C}_{u, v}^{2}$

$$
\begin{aligned}
& y_{1}^{2}-y_{2}^{2}=u \psi_{1}\left(y_{1}, \ldots, y_{4} ; u, v\right)+v \psi_{2}\left(y_{1}, \ldots, y_{4} ; u, v\right), \\
& y_{1} y_{2}-y_{3}^{2}=u \psi_{3}\left(y_{1}, \ldots, y_{4} ; u, v\right)+v \psi_{4}\left(y_{1}, \ldots, y_{4} ; u, v\right)
\end{aligned}
$$


by $\boldsymbol{\mu}_{4}$-action:

$y_{1} \mapsto-\mathrm{i} y_{1}, \quad y_{2} \mapsto \mathrm{i} y_{2}, \quad y_{3} \mapsto-y_{3}, \quad y_{4} \mapsto \mathrm{i} y_{4}, \quad u \mapsto \mathrm{i} u, \quad v \mapsto-\mathrm{i} v$ (as an example one can take $\psi_{1}=\psi_{4}=y_{4}, \psi_{2}=\psi_{3}=0$ ).

9.1.2 ([MP08a, 1.2.4]). $f$ is a $\mathbb{Q}$-conic bundle, $(X, C)$ is of type (ID $\left.{ }^{\vee}\right)$ at $P,(Z, o)$ is Du Val of type $\mathrm{A}_{1},(X, C)$ is a quotient of a Gorenstein conic bundle given by the following equation in $\mathbb{P}_{y_{1}, y_{2}, y_{3}}^{2} \times \mathbb{C}_{u, v}^{2}$

$$
y_{1}^{2}+y_{2}^{2}+\psi(u, v) y_{3}^{2}=0, \quad \psi(u, v) \in \mathbb{C}\left\{u^{2}, v^{2}, u v\right\}
$$

by $\boldsymbol{\mu}_{2}$-action:

$$
u \mapsto-u, \quad v \mapsto-v, \quad y_{1} \mapsto-y_{1}, \quad y_{2} \mapsto y_{2}, \quad y_{3} \mapsto y_{3}
$$

Here $\psi(u, v)$ has no multiple factors. In this case, $(X, P)$ is the only singular point and it is of type $\mathrm{cA} / 2$ or $\mathrm{cAx} / 2$.

9.1.3 ([MP08a, 1.2.5]). $f$ is a $\mathbb{Q}$-conic bundle, $(X, C)$ is of type (IA $\left.{ }^{\vee}\right)$ at $P$ with $\bar{m}=2, s=2,(Z, o)$ is Du Val of type $\mathrm{A}_{1},(X, P)$ is a cyclic quotient singularity of type $\frac{1}{4}(1,1,3)$, and $(X, C)$ is the quotient of the index-two $\mathbb{Q}$-conic bundle germ given by the following two equations in $\mathbb{P}(1,1,1,2)_{y_{1}, \ldots, y_{4}} \times \mathbb{C}_{u, v}^{2}$

$$
\begin{array}{ll}
y_{1}^{2}-y_{2}^{2} & =u \psi_{1}\left(y_{1}, \ldots, y_{4} ; u, v\right)+v \psi_{2}\left(y_{1}, \ldots, y_{4} ; u, v\right), \\
y_{3}^{2} & =u \psi_{3}\left(y_{1}, \ldots, y_{4} ; u, v\right)+v \psi_{4}\left(y_{1}, \ldots, y_{4} ; u, v\right)
\end{array}
$$

by $\boldsymbol{\mu}_{2}$-action:

$$
y_{1} \mapsto y_{1}, \quad y_{2} \mapsto-y_{2}, \quad y_{3} \mapsto y_{3}, \quad y_{4} \mapsto-y_{4}, \quad u \mapsto-u, \quad v \mapsto-v
$$

As an example one can take $\psi_{1}=\psi_{4}=y_{4}, \psi_{2}=0, \psi_{3}=u y_{2}^{2}+\lambda y_{1} y_{2}$, where $\lambda$ is a constant. If $\lambda \neq 0$, then $P$ is the only singular point. If $\lambda=0$, then $X$ has also a type (III) point.

9.1.4 ([MP08a, 1.2.6]). $f$ is a $\mathbb{Q}$-conic bundle, $(X, C)$ is of type $\left(\mathrm{II}^{\vee}\right)$ at $P,(Z, o)$ is Du Val of type $\mathrm{A}_{1}$, and $(X, C)$ is the quotient of the same form as in 9.1.3. As an example one can take $\psi_{1}=u^{2} y_{4}, \psi_{2}=\psi_{4}=y_{4}$, $\psi_{3}=u y_{2}^{2}+\lambda y_{1} y_{2}$, where $\lambda$ is a constant.

9.1.5 ([KM92, Theorem 4.11.2]). $f$ is divisorial, $(X, C)$ is of type $\left(\mathrm{II}^{\vee}\right)$ at $P$, a general member $H \in\left|\mathscr{O}_{X}\right|$ is normal. The graph $\Delta(H, C)$ is of the form

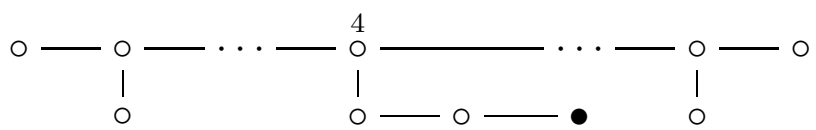

In this case $(X, C)$ is a quotient of an index two divisorial curve germ $(\bar{X}, \bar{C})$ by $\boldsymbol{\mu}_{2}$ that acts freely outside $P$ and switches two components of $\bar{C}$. The point $(Z, o)$ is terminal of index two given by

$$
\left\{t \phi\left(y_{1}, t\right)+y_{3}^{2}-y_{2}^{2}=0\right\} / \boldsymbol{\mu}_{2}(1,1,0,1)
$$


(cf. (8.2.17)) where the image of the exceptional divisor is the curve $\left\{y_{2}=y_{3}=t=0\right\} / \boldsymbol{\mu}_{2}$.

9.1.6 ([MP11, Theorem 1.9]). $f$ is birational, a general member $H \in$ $\left|\mathscr{O}_{X}\right|$ is normal and has only log terminal singularities of class $\mathrm{T}$ (see 9.1 .8 below). The graph $\Delta(H, C)$ is of the form

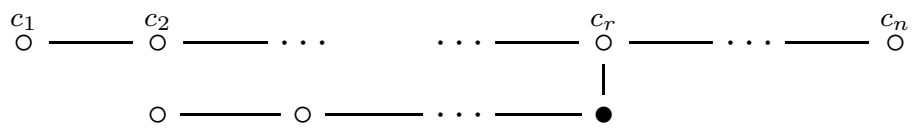

Here $r \neq 1, n$ and the chain $\left[c_{1}, \ldots, c_{n}\right]$ corresponds to the non-Du Val singularity $(H, P)$ of class $\mathrm{T}$. The chain of $(-2)$-vertices in the bottom line corresponds to a Du Val point $(H, Q)$. It is possible that this chain is empty (i.e., $(H, Q)$ is smooth). The germ $(X, C)$ is of type (IA $\left.{ }^{\vee}\right)$ and $C^{\sharp}$ explained in 9.4 is reducible. The contraction $f$ is divisorial or flipping according as $\left(H_{Z^{\prime}}^{\prime}, o^{\prime}\right)$ explained in 9.5 is Gorenstein or not.

9.1.8. Recall that a surface log terminal singularity $(H, P)$ is called a singularity of class $\mathrm{T}$, if it admits a one-parameter smoothing $\left\{H_{t}\right\}$, $H_{0}=H$ whose total space $X=\cup H_{t}$ is $\mathbb{Q}$-Gorenstein [LW86], [KSB88]. By the inversion of adjunction this total space must be terminal. Any singularity of class $\mathrm{T}$ is either $\mathrm{Du}$ Val or a cyclic quotient

$$
\frac{1}{m^{2} d}(1, m d t-1), \quad \operatorname{gcd}(m, t)=1 .
$$

There is an explicit characterization of such singularities in terms of minimal resolutions, see [KSB88, § 3] for details.

9.2. The rough idea of the proof of Theorem 9.1 is to apply the construction (4.6.2). Then $(X, C)$ can be viewed as a quotient of an extremal curve germ $\left(X^{\prime}, C^{\prime}\right)$ with reducible central fiber by $\boldsymbol{\mu}_{s}$. In the case $\left(\mathrm{ID}^{\vee}\right)$ we have $\bar{m}=1$. Hence, $\left(X^{\prime}, C^{\prime}\right)$ is a Gorenstein conic bundle germ [2.7.1](i), Then it is easy to write down the action explicitly Pro97, $\S 2$. Similarly, in the cases $\left(\mathrm{IE}^{\vee}\right)$ and $\left(\mathrm{II}^{\vee}\right)$ we have $\bar{m}=2$. Then $\left(X^{\prime}, C^{\prime}\right)$ is an extremal curve germ of index two and we can apply the results of Sect. 8. The case $\left(\mathrm{IC}^{\vee}\right)$ does not occur [Mor88, Th. 6.1(i)], [MP08a, 7.3].

9.3. Consider the case $\left(\mathrm{IA}^{\vee}\right)$. We need the following helpful observation which allows to study a general hyperplane section $H \in\left|\mathscr{O}_{X}\right|$. It will also be used below in the case (IA).

9.3.1. Lemma. Let $(Z, o)$ be a normal threefold singularity and let $D_{Z} \in$ $\left|-K_{X}\right|$ be a general member. Assume that $\left(D_{Z}, o\right)$ is a Du Val singularity of type A. Then for a general hyperplane section $H_{Z}$, the pair $\left(X, H_{Z}+\right.$ $\left.D_{Z}\right)$ is lc. In particular, $\left(H_{Z}, o\right)$ is a cyclic quotient singularity. 
Proof. Clearly, $H_{Z} \cap D_{Z}$ is general hyperplane section of $\left(D_{Z}, o\right)$ and so $H_{Z} \cap D_{Z}=\Gamma_{1}+\Gamma_{2}$ for some irreducible curves $\Gamma_{i}$ such that the pair $\left(D_{Z}, \Gamma_{1}+\Gamma_{2}\right)$ is lc. By the inversion of adjunction so is the pair $\left(Z, D_{Z}+H_{Z}\right)$ [Sho93, §3], [Kaw07]. Hence $\left(H_{Z}, \Gamma_{1}+\Gamma_{2}\right)$ is lc and $\left(H_{Z}, o\right)$ is a cyclic quotient singularity (see, e.g., [Kol92, Ch. 3]).

9.3.2. Proposition. Let $f:(X, C) \rightarrow(Z, o)$ be an extremal curve germ ( $C$ is not necessarily irreducible). Let $D \in\left|-K_{X}\right|$ and $H \in\left|\mathscr{O}_{X}\right|$ be general members. Let $\Lambda$ be the non-normal locus of $H$ and let $\nu: H^{\mathrm{n}} \rightarrow$ $H$ be the normalization (if $H$ is normal we put $\Lambda=\varnothing$ and $\nu=\mathrm{id}$ ).

Assume that $D \cap C$ is a point $P$ such that $(D, P)$ is a $D u$ Val singularity of type $\mathrm{A}$. Then the pairs $(X, D+H)$ and $\left(H^{\mathrm{n}}, \nu^{-1}(D)+\nu^{-1}(\Lambda)\right)$ are log canonical. In particular, $H$ has only normal crossings in codimension one. If $f$ is birational, then the pair $\left(Z, D_{Z}+H_{Z}\right)$ is also log canonical, where $D_{Z}=f(D) \in\left|-K_{Z}\right|$ and $H_{Z}:=f(H) \in\left|\mathscr{O}_{Z}\right|$. In this case, $\left(H_{Z}, o\right)$ is a cyclic quotient singularity.

Proof. First we consider the case where $f$ is birational. Then $\left(D_{Z}, o\right) \simeq$ $(D, P)$ is a Du Val singularity of type A. By Lemma 9.3.1 the pair $\left(X, H_{Z}+D_{Z}\right)$ is lc. Take $H:=f^{*} H_{Z}$. Then $K_{X}+D+H=f^{*}\left(K_{Z}+\right.$ $\left.D_{Z}+H_{Z}\right)$, i.e., the contraction $f$ is $K_{X}+D+H$-crepant. Hence the pair $(X, D+H)$ is lc and so is the pair $\left(H^{\mathrm{n}}, \nu^{-1}(D)+\nu^{-1}(\Lambda)\right)$ again by the inversion of adjunction.

Now consider the case where $Z$ is a surface. First we claim that $(X, D+H)$ is lc near $D$. Consider the restriction $\varphi=f_{D}:(D, P) \rightarrow$ $(Z, o)$. Let $\Xi \subset Z \simeq \mathbb{C}^{2}$ be the branch divisor of $\varphi$. By the Hurwitz formula we can write $K_{D}=\varphi^{*}\left(K_{Z}+\frac{1}{2} \Xi\right)$. Hence,

$$
K_{D}+\left.H\right|_{D}=\varphi^{*}\left(K_{Z}+\frac{1}{2} \Xi+H_{Z}\right) .
$$

Using this and the inversion of adjunction we get the following equivalences: $(X, D+H)$ is lc near $D \Longleftrightarrow\left(D,\left.H\right|_{D}=\varphi^{*} H_{Z}\right)$ is lc $\Longleftrightarrow$ $\left(Z=\mathbb{C}^{2}, \frac{1}{2} \Xi+H_{Z}\right)$ is lc. Thus it is sufficient to show that $\left(Z, \frac{1}{2} \Xi+H_{Z}\right)$ is lc. Let $\xi(u, v)=0$ be the equation of $\Xi \subset \mathbb{C}^{2}$. Then $(D, P)$ is given by the equation $w^{2}=\xi(u, v)$ in $\mathbb{C}_{u, v, w}^{3}$. By the classification of Du Val singularities we can choose coordinates $u, v$ so that $\xi=u^{2}+v^{n+1}$. Take $H_{Z}:=\{v-u=0\}$. Then $\left.\operatorname{ord}_{0} \xi(u, v)\right|_{H_{Z}}=2$. By the inversion of adjunction the pair $\left(Z, H_{Z}+\frac{1}{2} \Xi\right)$ is lc. Thus we have shown that $(X, D+H)$ is lc near $D$. Assume that $(X, D+H)$ is not lc at some point $Q \in C$. By the above, $Q \notin D$. Note that $H$ is smooth outside $C$ by Bertini's theorem. If $H$ is normal, then we have an immediate contradiction by a connectedness result [Sho93, Th. 6.9] applied to $\left(H,\left.D\right|_{H}\right)$. If $H$ is not normal, we can apply the same result on the normalization.

9.3.3. We claim that $H$ is normal. Assume the converse, i.e. $H$ is singular along $C$. The lemma above implies that in our situation $C$ is the 
minimal $\log$ canonical center of $(X, H)$ [Kaw97]. Now let $\tau:\left(X^{\prime}, C^{\prime}\right) \rightarrow$ $(X, C)$ be the torsion free cover 4.6 and let $H^{\prime}:=\tau^{*} H$. Then the pair $\left(X^{\prime}, C^{\prime}\right)$ is $\log$ canonical and $C^{\prime}$ is its minimal log canonical center [Kol92, 20.4]. Since the minimal log canonical center is normal [Kaw97], we conclude that $C^{\prime}$ is irreducible. This contradicts imprimitivity of $(X, C)$ at $P$.

9.3.4. Thus $H$ is normal and then $P$ is the only log canonical center of the pair $(X, H+D)$. This implies that the pair $(X, H)$ is plt. Since $H$ is a Cartier divisor, the singularities of $H$ are of class $\mathrm{T}$ (see 9.1.8). This gives very strong restriction to the dual graph of the minimal resolution. If $(X, C)$ is a $\mathbb{Q}$-conic bundle germ, then using completely combinatorial techniques one can show that for $\Delta(H, C)$ there is only one possibility (cf. [Pro04]):

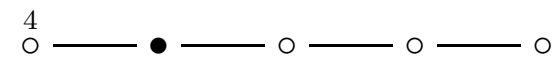

But in this case the pair $(H, C)$ is plt, hence so is $\left(H^{\prime}, C^{\prime}\right)$, whence $C^{\prime}$ cannot split and this case does not occur. In the birational case we obtain (9.1.7). Since $(H, C)$ cannot be plt as above, we have $r \neq 1, n$. This concludes the explanation of the proof of Theorem 9.1 ,

9.4. To decide whether an extremal curve germ $(X, C)$ is locally imprimitive at $P$, one needs to compute the inverse image $C^{\sharp}$ of $C$ in the index-one cover $\left(X^{\sharp}, P^{\sharp}\right)$ which can be computed within $H^{\sharp}$ the pull back of $H$ as in 9.6, once the diagram like (9.1.7) is exhibited. Indeed, $P$ is imprimitive if and only if the splitting degree $s>1$, which is equal to the number of irreducible components of $C^{\sharp}$, see 4.2 .

9.5. To distinguish divisorial and flipping contractions in the case 9.1.6 one can use the following arguments. Let $f^{\prime}:\left(X^{\prime}, C^{\prime}\right) \rightarrow\left(Z^{\prime}, o^{\prime}\right)$ be the torsion free cover (4.6.2). By 6.5 the germ $\left(X, C \simeq \mathbb{P}^{1}\right)$ is divisorial if and only if the point $(Z, o)$ is terminal and if and only if the point $\left(Z^{\prime}, o^{\prime}\right)$ is terminal of index one (i.e. isolated cDV). Note that in our case $\left(H_{Z}, o\right)$ is a cyclic quotient singularity and so is its pull-back $\left(H_{Z}^{\prime}, o^{\prime}\right)$. Hence the divisoriality of $(X, C)$ is equivalent to that $\left(H_{Z}^{\prime}, o^{\prime}\right)$ is Gorenstein, that is, Du Val singularity in our case. Once $\left(H, C \simeq \mathbb{P}^{1}\right)$ is given, one can find its splitting cover $\left(H^{\prime}, C^{\prime}\right)$ and so the surface germ $\left(H_{Z}^{\prime}, o^{\prime}\right)$ can be computed.

9.6. Example. Consider the quotient surface singularity

$$
(H, P)=\left(\mathbb{C}_{u, v}^{2}, 0\right) / \boldsymbol{\mu}_{m^{2}}(1, m-1), \quad m \geq 3 .
$$

It is of class $\mathrm{T}$ and for its index-one cover we have

$$
\left(H^{\sharp}, P^{\sharp}\right)=\left(\mathbb{C}^{2}, 0\right) / \boldsymbol{\mu}_{m}(1, m-1) .
$$


Hence it is Du Val of type $\mathrm{A}_{\mathrm{m}-1}$. Consider the $\boldsymbol{\mu}_{m}$-equivariant curve

$$
C^{\sharp}=\left\{u^{m-2}-v^{m+2}=0\right\} / \boldsymbol{\mu}_{m} \subset H^{\sharp}
$$

and $C=C^{\sharp} / \boldsymbol{\mu}_{m}$. Then $C^{\sharp}$ is irreducible (resp. has two irreducible components) if $m$ is odd (resp. $m$ is even) and it is easy to see that $C$ is smooth. Now consider the weighted $\frac{1}{m^{2}}(1, m-1)$-blowup of $(H, P)$. In the chart $v \neq 0$ the origin is a Du Val point $\mathbb{C}^{2} / \boldsymbol{\mu}_{m-1}\left(-1, m^{2}\right)$ of type $\mathrm{A}_{\mathrm{m}-2}$, the exceptional divisor $\Lambda$ is $v^{\prime}=0$, and the proper transform $\hat{C}$ of $C$ is given by $v^{\prime}=u^{\prime m-2}$. Hence, on the minimal resolution of the $\mathrm{A}_{\mathrm{m}-2}$-point, both $\Lambda$ and $\hat{C}$ meet the same end of the chain. Therefore, the dual graph $\Delta(H, C)$ is of the form

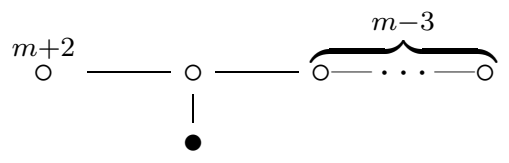

Now suppose that $C$ is a compact curve, $C \simeq \mathbb{P}^{1}$ and consider a surface germ $(H, C)$ whose minimal resolution has the above form. It is easy to see that $K_{H} \cdot C=-2 / m$ and $C$ can be contracted to a cyclic quotient singularity $\left(H_{Z}, o\right)$ of type $\frac{1}{4}(1,1)$. There is a Gorenstein threefold germ $X^{\sharp}$ with $\boldsymbol{\mu}_{m}$-action containing $H^{\sharp}$ as a $\boldsymbol{\mu}_{m}$-stable hypersurface. According to 6.6 (and [KSB88, § 3]) the germ $(H, C)$ has a smoothing in a $\mathbb{Q}$-Gorenstein family. Thus there exists a $\mathbb{Q}$-Gorenstein threefold $X$ containing $H$ as a Cartier divisor. By the inversion of adjunction (see [Sho93, § 3], Kol92, Ch. 17]) $X$ has only terminal singularities. By arguments similar to 6.3 we see that there exists a birational contraction $f: X \rightarrow Z$ extending $H \rightarrow H_{Z}$. We note that $C^{\sharp}$ can be identified with the pull back of $C$ by the splitting cover of $(X, C)$ at $P$. Now we distinguish two cases according to the parity of $m$.

a) $m$ is even. Then $(X, C)$ is imprimitive of splitting degree 2 at $P$. Since $\left(H_{Z}, o\right)$ is a type T singularity of index 2, its pull-back $\left(H_{Z}^{\prime}, o^{\prime}\right)$ in the torsion free (degree 2) cover (4.6.2) is Du Val and so $\left(Z^{\prime}, o^{\prime}\right)$ is a $\mathrm{cDV}$ point. Hence, both contractions $f^{\prime}$ and $f$ are divisorial.

b) $m$ is odd. Then $(X, C)$ is primitive and the contraction is flipping by (4.7.5). Note that in this case the singularity $(Z, o)$ is not $\mathbb{Q}$-Gorenstein. On the other hand, since $\left(H_{Z}, o\right)$ is a singularity of class $\mathrm{T}$, it has a $\mathbb{Q}$-Gorenstein smoothing. This smoothing belongs to a component of the versal deformation space which is different from that corresponding to $(Z, o)$ [KSB88, 3.9].

10. Cases (IC) And (IIB)

In this section we consider curve germs of types (IC) and (IIB). 
10.1. Case (IIB). Let $(X, P)$ be the germ of a three-dimensional terminal singularity and let $C \subset(X, P)$ be a smooth curve. Recall that the triple $(X, C, P)$ is said to be of type (IIB) if $(X, P)$ is a terminal singularity of type cAx/4 and there are analytic isomorphisms

$$
\begin{aligned}
(X, P) & \simeq\left\{y_{1}^{2}-y_{2}^{3}+\alpha=0\right\} / \boldsymbol{\mu}_{4} \subset \mathbb{C}_{y_{1}, \ldots, y_{4}}^{4} / \boldsymbol{\mu}_{4}(3,2,1,1), \\
C & =\left\{y_{1}^{2}-y_{2}^{3}=y_{3}=y_{4}=0\right\} / \boldsymbol{\mu}_{4},
\end{aligned}
$$

where $\alpha=\alpha\left(y_{1}, \ldots, y_{4}\right) \in\left(y_{3}, y_{4}\right)$ is a semi-invariant with $\mathrm{wt}(\alpha) \equiv 2$ mod 4 and the quadratic part $\alpha_{2}$ of $\alpha\left(0,0, y_{3}, y_{4}\right)$ is not zero (see Mor88, A.3]). We say that $(X, P)$ is a simple (resp. double) cAx/4-point if $\operatorname{rk} \alpha_{2}=2\left(\right.$ resp. $\left.\operatorname{rk} \alpha_{2}=1\right)$.

10.2. Theorem ([MP14). Let $f:\left(X, C \simeq \mathbb{P}^{1}\right) \rightarrow(Z, o)$ be an extremal curve germ. Suppose that $X$ contains a point $P$ of type (IIB). Then $(X, C)$ is not flipping [KM92, Th. 4.5] and $P \in X$ is the unique singular point of $X$ on $C$. Furthermore, a general member $H \in\left|\mathscr{O}_{X}\right|$ is normal, smooth outside $P$, and has only rational singularities. The following are

\begin{tabular}{|c|c|c|c|}
\hline No. & $\mathrm{cAx} / 4$-point & $\Delta(H, C)$ & $\Delta\left(H_{Z}, o\right)$ \\
\hline 10.2 .1 & simple & 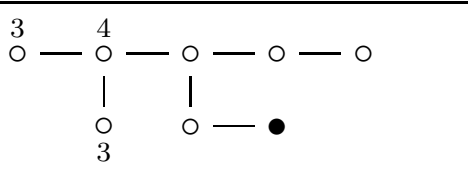 & $\mathrm{A}_{2}$ \\
\hline 10.2 .2 & simple & 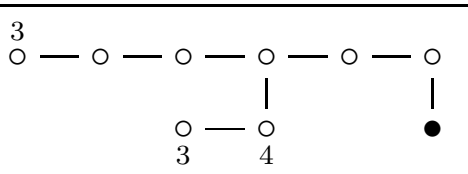 & $\mathrm{A}_{0}$ \\
\hline 10.2 .3 & double & 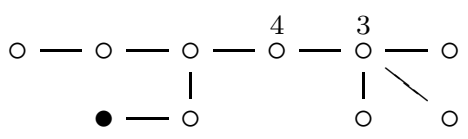 & $\mathrm{D}_{4}$ \\
\hline 10.2 .4 & double & $\begin{array}{cc}\circ- & \stackrel{3}{\circ}-\circ-\circ-0-\circ-\circ \\
1 & ! \\
0 & 0 \\
4\end{array}$ & \\
\hline
\end{tabular}
the only possibilities for the dual graphs of $(H, C)$ and $H_{Z}:=f(H)$ :

The last column indicates if the germ is divisorial (d) or $\mathbb{Q}$-conic bundle (c); and the column $\Delta\left(H_{Z}, o\right)$ is not used in the latter case (c).

An example of divisorial contraction of type 10.2.1] is given in [KM92, 4.12]. The case 10.2.2 was studied also by T. Ducat [Duc16, Thm. $4.1(2 \mathrm{~b})]$ in terms of symbolic blowups of smooth threefolds.

Sketch of the proof. In our case a general member $D \in\left|-K_{X}\right|$ contains $C$, has only Du Val singularities, and the graph $\Delta(D, C)$ has the form 7.4.3. Under the identifications of 10.1, a general member $D \in\left|-K_{X}\right|$ 
near $P$ is given by $\lambda y_{3}+\mu y_{4}=0$ for some $\lambda, \mu \in \mathscr{O}_{X}$ such that $\lambda(0)$, $\mu(0)$ are general in $\mathbb{C}^{*}[\mathrm{KM} 92,2.11],[\mathrm{MP} 09, \S 4]$. Let $\Gamma:=H \cap D$.

By [KM92, Th. 4.5] the contraction $f$ is not flipping. If $f$ is divisorial, we put $D_{Z}:=f(D)$ and $\Gamma_{Z}:=f(\Gamma)$. Then $D_{Z} \in\left|-K_{Z}\right|, H_{Z}$ is a general hyperplane section of $(Z, o)$, and $\Gamma_{Z}$ is a general hyperplane section of $D_{Z}$. If $f$ is a $\mathbb{Q}$-conic bundle, we put $D_{Z}:=\operatorname{Spec}_{Z} f_{*} \mathscr{O}_{D}$ (the Stein factorization) and let $\Gamma_{Z} \subset D_{Z}$ be the image of $\Gamma$. In both cases $D_{Z}$ is a Du Val singularity of type $\mathrm{E}_{6}$ by 7.4 .3 .

We claim that $\Gamma=C+\Gamma_{1}$ (as a scheme), where $\Gamma_{1}$ is a reduced irreducible curve, and $H$ is normal, smooth outside $P$, and has only rational singularities. Consider two cases:

10.2.5. Case: $f$ is divisorial. Since the point $(Z, o)$ is terminal of index 1 , the germ $\left(H_{Z}, o\right)$ is a Du Val singularity. Since $\Gamma_{Z}$ is a general hyperplane section of $D_{Z}$ we see that the graph $\Delta(D, \Gamma)$ has the form

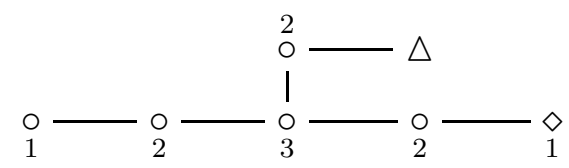

where $\Delta$ corresponds to the proper transform of $\Gamma_{Z}$ and numbers attached to vertices are coefficients of corresponding exceptional curves in the pull-back of $\Gamma_{Z}$. By Bertini's theorem $H$ is smooth outside $C$. Since the coefficient of $C$ equals $1, D \cap H=C+\Gamma_{1}$ (as a scheme), so $H$ is smooth outside $P$. In particular, $H$ is normal. Since $f_{H}: H \rightarrow H_{Z}$ is a birational contraction and $\left(H_{Z}, o\right)$ is a Du Val singularity, the singularities of $H$ are rational.

10.2.7. Case: $f$ is a $\mathbb{Q}$-conic bundle. We may assume that, in a suitable coordinate system, the germ $\left(D_{Z}, o_{Z}\right)$ is given by $x^{2}+y^{3}+z^{4}=0$ and the double cover $\left(D_{Z}, o_{Z}\right) \longrightarrow(Z, o)$ is just the projection to the $(y, z)$-plane. Then $\Gamma_{Z}$ is given by $z=0$. As in the case above we see that the graph $\Delta(D, \Gamma)$ has the form (10.2.6). Therefore, $H$ is smooth outside $P$. The restriction $f_{H}: H \rightarrow H_{Z}$ is a rational curve fibration. Hence $H$ has only rational singularities. This proves our claim.

10.2.8. Further, $\operatorname{gr}_{C}^{1} \mathscr{O} \simeq \mathscr{O}_{\mathbb{P}^{1}}\left(d_{1}\right) \oplus \mathscr{O}_{\mathbb{P}^{1}}\left(d_{2}\right)$ for some $d_{1} \geq d_{2}$. Since $H^{1}\left(\operatorname{gr}_{C}^{1} \mathscr{O}\right)=0$ by Corollary 2.4.1](i)], we have $d_{2} \geq-1$. Since $H$ is normal, we have $d_{1} \geq 0$ (see Lemma 3.5.1). On the other hand, $\operatorname{deg} \operatorname{gr}_{C}^{1} \mathscr{O}=1-i_{P}(1)$ by $(3.1 .3)$. One can compute $i_{P}(1)=2$ from [Mor88, (2.12)] for $P$ of type (IIB) described in 10.1. Therefore,

$$
\operatorname{gr}_{C}^{1} \mathscr{O} \simeq \mathscr{O}_{\mathbb{P}^{1}} \oplus \mathscr{O}_{\mathbb{P}^{1}}(-1)
$$

and $\mathscr{O}_{C}(-H)=\mathscr{O} \subset \operatorname{gr}_{C}^{1} \mathscr{O}$, i.e. the local equation of $H$ must be a generator of $\mathscr{O} \subset \operatorname{gr}_{C}^{1} \mathscr{O}$. In the notation of 10.1 the surface $H \subset X$ is locally near $P$ given by the equation $y_{3} v_{3}+y_{4} v_{4}=0$, where $v_{3}, v_{4} \in$ 
$\mathscr{O}_{P^{\sharp}, X^{\sharp}}$ are semi-invariants with $\operatorname{wt}\left(v_{i}\right) \equiv 3$ and at least one of $v_{3}$ or $v_{4}$ contains a linear term in $y_{1}$. Therefore, the surface germ $(H, P)$ can be given in $\mathbb{C}^{4} / \boldsymbol{\mu}_{4}(3,2,1,1)$ by two equations:

$$
\begin{aligned}
y_{1}^{2}-y_{2}^{3}+\eta\left(y_{3}, y_{4}\right)+\phi\left(y_{1}, y_{2}, y_{3}, y_{4}\right) & =0, \\
y_{1} l\left(y_{3}, y_{4}\right)+y_{2} q\left(y_{3}, y_{4}\right)+\xi\left(y_{3}, y_{4}\right)+\psi\left(y_{1}, y_{2}, y_{3}, y_{4}\right) & =0,
\end{aligned}
$$

where $\eta, l, q$ and $\xi$ are homogeneous polynomials of degree 2, 1, 2 and 4, respectively, $\eta \neq 0, l \neq 0, \phi, \psi \in\left(y_{3}, y_{4}\right), \sigma$ - ord $\phi \geq 3 / 2, \sigma$ - ord $\psi \geq 2$. Moreover, $\operatorname{rk} \eta=2$ (resp. $\operatorname{rk} \eta=1)$ if $(X, P)$ is a simple (resp. double) cAx/4-point. Then considering the weighted $\frac{1}{4}(3,2,1,1)$-blowup and using rationality of $(H, P)$, as well as, generality of $H$ in $\left|\mathscr{O}_{X}\right|$ one can obtain the possibilities in Theorem 10.2. See [MP14, § 3] for details.

10.3. Case $(\mathrm{IC})$. Let $\left(X, C \simeq \mathbb{P}^{1}\right)$ be an extremal curve germ. Assume that $(X, C)$ has a type (IC) point $P$ of index $m$. Then $P$ is the only singular point of $X, m$ is odd $\geq 5$, and $w_{p}(0)=(m-1) / m$. Moreover, $i_{P}(1)=a_{1}=2$ and

$$
(X, C, P) \simeq\left(\mathbb{C}_{y_{1}, y_{2}, y_{4}}^{3},\left\{y_{1}^{m-2}-y_{2}^{2}=y_{4}=0\right\}, 0\right) / \boldsymbol{\mu}_{m}(2, m-2,1) .
$$

(see Lemma 5.7](i) and [Mor88, 5.5, 6.5, A.3]). Below is a complete classification of extremal curve germs of type (IC):

10.4. Theorem ([KM92, §8], [MP14]). Let $f:\left(X, C \simeq \mathbb{P}^{1}\right) \rightarrow(Z, o)$ be an extremal curve germ of type (IC). Let $P \in X$ be (a unique) singular point and let $m$ be its index. Then a general member $H \in\left|\mathscr{O}_{X}\right|$ is normal, smooth outside $P$, has only rational singularities. Moreover, $(X, C)$ is not divisorial and we have one of the following:

10.4.1. $(X, C)$ is flipping and the following are the only possibilities for the dual graphs of $(H, C)$ and $H_{Z}=f(H)$ :

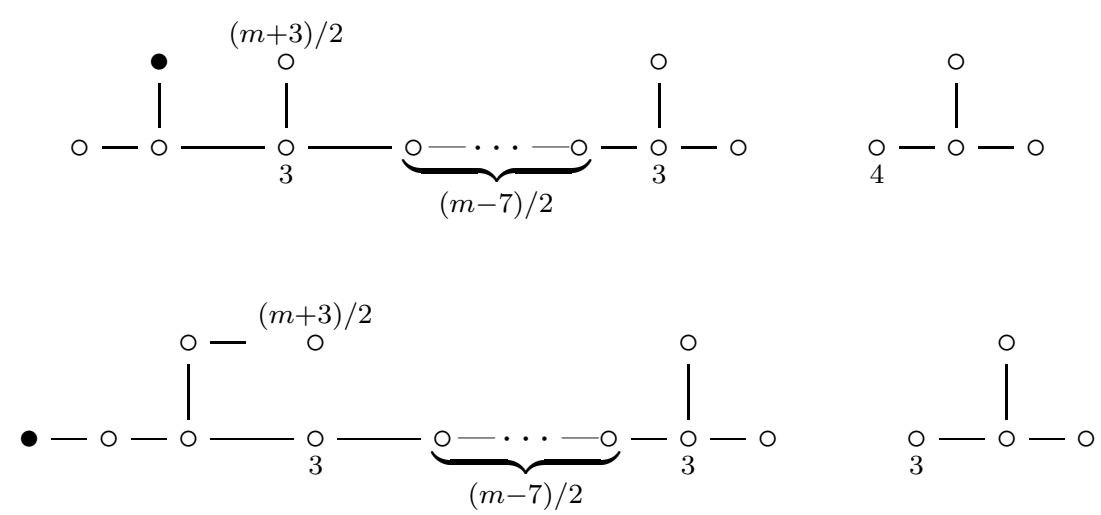

where ${ }_{3}^{\circ}-\underbrace{\mathrm{a}-\cdots-\cdots}_{(m-7) / 2}-{ }_{3}^{\circ}$ must be replaced with ${ }_{4}^{\circ}$ in the case $m=5$. 
10.4.2. $(X, C)$ is a $\mathbb{Q}$-conic bundle, $m=5$, and $\Delta(H, C)$ has the form:

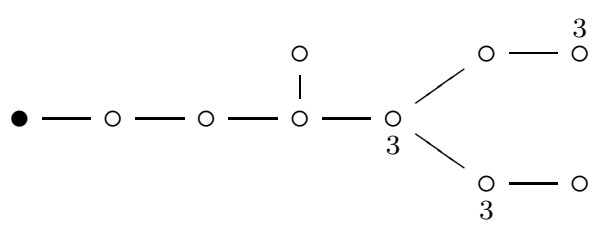

In the flipping case, the general member $H^{+} \in\left|\mathscr{O}_{X^{+}}\right|$of the flipped variety is also computed. Here $X^{+}$is either of index two or Gorenstein [KM92, A.3].

\section{CASE (IA)}

11.1. An extremal curve germ $\left(X, C \simeq \mathbb{P}^{1}\right)$ is said to be of type (IA) if it contains exactly one non-Gorenstein point $P$ which is of type (IA). For readers' convenience, we note the following characterization for an extremal curve germ $\left(X, C \simeq \mathbb{P}^{1}\right)$ to be of type (IA) with $D \in\left|-K_{X}\right|$ a general member (see 5.4 and 7.4 ):

$(X, C)$ is of type (IA) if and only if (i) $P$ is locally primitive, (ii) $D \cap C$ is a single point, and (iii) $(X, P)$

is not of type $\mathrm{cAx} / 4$.

11.1.1. From now on we assume that the germ $\left(X, C \simeq \mathbb{P}^{1}\right)$ satisfies the assumptions of 11.1. The following are the only possibilities for the singularity $(X, P)$ :

(i) $(X, P)$ is of type $\mathrm{cA} / \mathrm{m}$, in this case $(X, C)$ is said to be of type $(\mathrm{k} 1 \mathrm{~A})$ according to KM92;

(ii) $(X, P)$ is of type $\mathrm{cD} / 3$;

(iii) $(X, P)$ is of type $\mathrm{cAx} / 2, \mathrm{cD} / 2$ or $\mathrm{cE} / 2$.

Thus in our case of type (IA), $(X, C)$ is semistable if and only if it is of type (k1A). Extremal curve germs of index two are classified in Sect. 8 , Thus we discuss here cases $(\mathrm{k} 1 \mathrm{~A})$ and $\mathrm{cD} / 3$. We start with $\mathbb{Q}$-conic bundles:

11.2. Theorem ([MP11, 1.6]). Let $\left(X, C \simeq \mathbb{P}^{1}\right)$ be a $\mathbb{Q}$-conic bundle germ of index $m>2$ and of type (IA). Let $P \in X$ be the non-Gorenstein point. Then $(X, P)$ is a point of type $\mathrm{cA} / \mathrm{m}$ and a general member $H \in\left|\mathscr{O}_{X}\right|$ is not normal. Furthermore, the dual graph of $\left(H^{\mathrm{n}}, C^{\mathrm{n}}\right)$, the normalization $H^{\mathrm{n}}$ and the inverse image $C^{\mathrm{n}}$ of $C$, has the form:

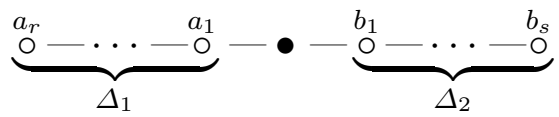

(in particular, $C^{\mathrm{n}}$ is irreducible). Here the chain $\Delta_{1}$ (resp., $\left.\Delta_{2}\right)$ corresponds to the singularity of type $\frac{1}{m}(1, a)$ (resp., $\frac{1}{m}(1,-a)$ ) for some integer a $(1 \leq a<m)$ relatively prime to $m$. The germ $(H, C)$ is 
analytically isomorphic to the germ along the line $y=z=0$ of the hypersurface given by the following weighted polynomial of degree $2 m$ in variables $x, y, z, u$ :

$$
\phi:=x^{2 m-2 a} y^{2}+x^{2 a} z^{2}+y z u
$$

in $\mathbb{P}(1, a, m-a, m)$. Furthermore $(X, C)$ is given as an analytic germ of a subvariety of $\mathbb{P}(1, a, m-a, m) \times \mathbb{C}_{t}$ along $C \times 0$ given by

$$
\phi+\alpha_{1} x^{2 m-a} y+\alpha_{2} x^{m-a} u y+\alpha_{3} x^{2 m}+\alpha_{4} x^{m} u+\alpha_{5} u^{2}=0
$$

for some $\alpha_{1}, \ldots \alpha_{5} \in t \mathscr{O}_{0, \mathbb{C}_{t}}$ and there is a $\mathbb{Q}$-conic bundle structure $X \rightarrow \mathbb{C}^{2}$ through which the second projection $X \rightarrow \mathbb{C}_{t}$ factors.

11.3. Theorem ([MP11, 1.9], see also [Tzi05b]). Let $(X, C)$ be a birational extremal curve germ of type $(\mathrm{k} 1 \mathrm{~A})$. Let $P \in X$ be the point of index $m \geq 2$.

11.3.1. If a general element $H$ is normal, then the graph $\Delta(H, C)$ has the same form as in (9.1.7), however the cases $r=1$ and $r=n$ are not excluded.

11.3.2. If every member of $\left|\mathscr{O}_{X}\right|$ is non-normal, then the dual graph of the normalization $\left(H^{\mathrm{n}}, C^{\mathrm{n}}\right)$ is of the form

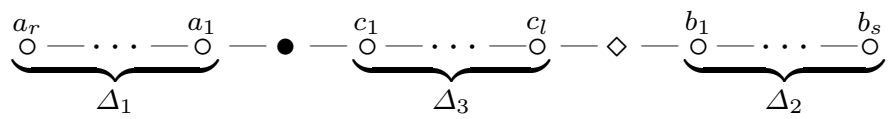

(in particular, $C^{\mathrm{n}}$ is reducible). The chain $\Delta_{1}$ (resp., $\left.\Delta_{2}\right)$ corresponds to the singularity of type $\frac{1}{m}(1, a)$ (resp., $\frac{1}{m}(1,-a)$ ) for some a with $\operatorname{gcd}(m, a)=1$ and the chain $\Delta_{3}$ corresponds to the point $\left(H^{\mathrm{n}}, Q^{\mathrm{n}}\right)$, where $Q^{\mathrm{n}}=C_{1}^{\mathrm{n}} \cap C_{2}^{\mathrm{n}}$. Moreover,

$$
\sum\left(c_{i}-2\right) \leq 2 \quad \text { and } \quad \widetilde{C}_{1}^{2}+\widetilde{C}_{2}^{2}+5-\sum\left(c_{i}-2\right) \geq 0
$$

where $\widetilde{C}=\widetilde{C}_{1}+\widetilde{C}_{2}$ is the proper transform of $C$ on the minimal resolution $\widetilde{H}$. Both components of $\widetilde{C}$ are contracted on the minimal model of $\widetilde{H}$. In this case,

$$
(X, C, P) \simeq\left(\left\{\alpha\left(x_{1}, \ldots, x_{4}\right)=0\right\}, x_{1} \text {-axis }, 0\right) / \boldsymbol{\mu}_{m}(1, a,-a, 0),
$$

where $\operatorname{gcd}(m, a)=1$ and $\alpha=0$ is the equation of a terminal $\mathrm{cA} / \mathrm{m}$-point in $\mathbb{C}^{4} / \boldsymbol{\mu}_{m}$. (In particular, $(X, C)$ is of type (IA)).

Conversely, for any germ $\left(H, C \simeq \mathbb{P}^{1}\right)$ of the form 11.3 .1 or 11.3 .2 admitting a birational contraction $(H, C) \rightarrow\left(H_{Z}, o\right)$ there exists a threefold birational contraction $f:(X, C) \rightarrow(Z, o)$ as in 11.1 of type (IA) such that $H \in\left|\mathscr{O}_{X}\right|$. 
11.3.4. To study a general member $H \in\left|\mathscr{O}_{X}\right|$ we can use Lemma 9.3.2. However we cannot assert as in 9.3 .3 that $H$ is normal. In fact, arguments similar to 9.3 .4 show that the case of normal $H$ does not occur if $(X, C)$ is a $\mathbb{Q}$-conic bundle.

11.4. Let us outline the proofs of Theorems 11.2 and 11.3. The case where $H$ is normal is teated in the same way as 9.1.6 (see 9.3.4) and $X$ can be recovered as a one-parameter deformation space by [6.6. Examples are given in 11.4.7 below.

Suppose that $H$ is not normal. Let $\nu: H^{\mathrm{n}} \rightarrow H$ be the normalization and let $C^{\mathrm{n}} \subset H^{\mathrm{n}}$ be the inverse image of $C$. By the inversion of adjunction the pair $(H, C)$ is slc, the pair $\left(H^{\mathrm{n}}, C^{\mathrm{n}}\right)$ is lc, and the point $P \in(H, C)$ is slt [Kol92, 16.9]. In particular, $H$ is a generically normal crossing divisor. At certain (finite number) of dissident points $H$ may have singularities worse than just normal crossing points.

11.4.1. Since $H$ has $\mathbb{Q}$-Gorenstein smoothing, by KSB88, Theorem 4.24, 5.2] the only possibilities are:

- Pinch point: $\left\{x^{2}-y^{2} z=0\right\} \subset \mathbb{C}^{3}$.

- Degenerate cusp of embedding dimension at most 4 , where a degenerate cusp is a non-normal Gorenstein singularity having a semi-resolution whose exceptional divisor is a cycle of smooth rational curves or a rational nodal curve (see [SB83]).

- Slt singularity of the form

$$
\left.\{x y=0\} / \boldsymbol{\mu}_{m}(a,-a, 1), \quad \operatorname{gcd}(a, n)=1\right\}
$$

(this point corresponds to $P \in H$ ).

11.4.2. The restriction $\nu_{C}: C^{\mathrm{n}} \rightarrow C$ of the normalization to the inverse image of $C$ is a double cover. We distinguish two possibilities:

(i) $C^{\mathrm{n}}$ is smooth irreducible and $\nu_{C}$ is branched at two points,

(ii) $C^{\mathrm{n}}$ has two irreducible components meeting at one point and the restriction of $\nu_{C}$ to each of them is an isomorphism.

A detailed analysis (see [MP11] and also [Tzi05b]) shows that(i) leads to the $\mathbb{Q}$-conic bundle case (11.2.1) while (ii) leads to the birational case (11.3.3). In both cases the subgraphs $\Delta_{1}$ and $\Delta_{2}$ correspond to points $P_{1}^{n}, P_{2}^{\mathrm{n}} \in H^{\mathrm{n}}$ lying over $P \in H$.

11.4.3. To recover $X$ as a one-parameter deformation space we also can apply arguments as in 6.6. However, in the case of non-normal surface $H$, it needs some restriction to singularities and additional technical tools [Tzi09]. Fortunately, the results of [Tzi09] are applicable if $H$ has singularities described above. Moreover, the miniversal deformation family of $(H, C)$ in the $\mathbb{Q}$-conic bundle case is computed explicitly [MP11, 6.8.3]. 
11.4.4. To check divisoriality one can use the criterion 6.5. Indeed, if $f$ is divisorial, then $(Z, o)$ is a terminal point and its index equals 1 because $(X, C)$ is primitive (see 4.5). Therefore, its general hyperplane section $\left(H_{Z}, o\right)$ must be a Du Val singularity. If on the contrary $f$ is flipping, then $(Z, o)$ is not $\mathbb{Q}$-Gorenstein and $\left(H_{Z}, o\right)$ cannot be Du Val. Given a graph $\Delta(H, C)$ of type (9.1.7) one can easily draw the graph $\Delta\left(H_{Z}\right)$ contracting black vertices successfully. Thus the Du Val condition of $\left(H_{Z}, o\right)$ can be checked in purely combinatorial terms.

11.4.5. Remark. Assume that in the assumptions of 11.3.1 and (9.1.7) we have $r=1$ or $r=n$. Then the graph $\Delta(H, C)$ is a chain. In this case there exists an element $D \in\left|-K_{X}\right|$ containing $C$ and having Du Val singularities only. This is a particular case of the situation considered in Mor02] where a powerful algorithm to construct $(X, C)$ was obtained.

11.4.6. One special case of Theorem 11.3 was studied in details in [HTU17]. There the authors assumed that the nearby fiber $H_{t}$ of the one-parameter deformation $\cup H_{t}=X$ has $b_{2}\left(H_{t}\right)=1$. This strong assumption is equivalent to that $H$ is normal and has so-called Wahl singularity at $P:(H, P) \simeq \mathbb{C}^{2} / \boldsymbol{\mu}_{m^{2}}(1, m a-1)$. Under this assumption, it is shown that birational germs of this type belong to the same deformation family as those of (k2A) studied in [Mor02], constructed the universal family, and the algorithm [Mor02] of computing flips was extended.

11.4.7. Examples. Consider several examples of extremal germs of type 11.3.1;

(i) The index two germs 8.2.1 8.2.5 are of type (IA). By using arguments of 11.4.4 one can conclude that the germ as in 8.2.1 is flipping and those in 8.2.2 8.2.5 are divisorial.

(ii) Let $\Delta(H, C)$ be of the form

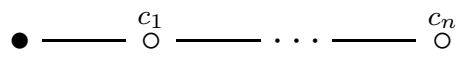

where the white vertices form a dual graph of a non-Du Val T-singularity (see 9.1.8 ). It is easy to see that $C$ can be contracted to a cyclic quotient non-Du Val point. Therefore, the one-parameter deformation produces a flipping contraction. Since $(H, C)$ is plt, the contraction is primitive.

(iii) Let $\Delta(H, C)$ be of the form

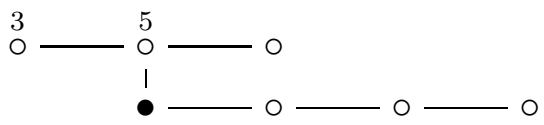

This is an example of a divisorial contraction to a smooth point.

(iv) A series of examples were given in $9.6 \mathrm{~b}$ ). 
For completeness, we provide an example of birational curve germ with non-normal $H$.

11.4.8. Example ([Tzi05b, Ex. 2], [MP11, Ex. 6.10.3]). Consider a surface $\tilde{H}$ containing a configuration with the following graph

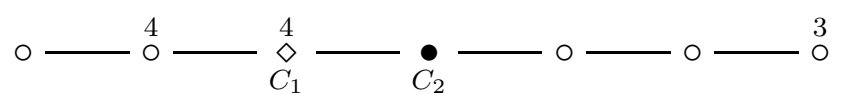

Contracting all curves except those marked by $C_{1}$ and $C_{2}$, we obtain a normal surface $H^{n}$ having two cyclic quotient singularities $P_{1}$ and $P_{2}$ of types $\frac{1}{7}(1,2)$ and $\frac{1}{7}(1,-2)$. Identifying the curves $C_{1}$ and $C_{2}$ we obtain a non-normal surface $H$ so that the map $\nu: H^{n} \rightarrow H$ is the normalization. The dissident singularities of $H$ are a degenerate cusp of multiplicity 2 and embedding dimension 3 at $\nu\left(C_{1} \cap C_{2}\right)$, and one point of type $\{x y=0\} / \boldsymbol{\mu}_{7}(2,-2,1)$. The results of [Tzi09] are applicable here and so there exists a one-parameter smoothing $X \supset H \supset C$ which is a divisorial curve germ and $H$ is general in $\left|\mathscr{O}_{X}\right|$, see [MP11, Prop. 6.3 and Th. 6.10].

11.5. Points of type $\mathrm{cD} / 3$. Let $(X, C, P)$ be a triple of type (IA), where $(X, P)$ is a singularity of type $\mathrm{cD} / 3$ [Mor85], Rei87]. These triples are described as follows (see [KM92, 6.5]). Put $\sigma:=(1,1,2,3)$. Up to coordinate change the point $(X, C, P)$ is given in $\mathbb{C}_{y_{1}, \ldots, y_{4}}^{4}$ as follows

$$
\begin{aligned}
& (X, C, P)=\left(\{\alpha=0\},\left\{y_{1} \text {-axis }\right\}, 0\right) / \boldsymbol{\mu}_{3}(1,1,2,0), \\
& \alpha=y_{4}^{2}+y_{3}^{3}+\delta_{3}\left(y_{1}, y_{2}\right)+(\text { terms of degree } \geq 4),
\end{aligned}
$$

where $\delta_{3} \neq 0$ is homogeneous of degree 3 and $\alpha$ is invariant. Moreover,

$$
\alpha \equiv y_{1}^{\ell} y_{i} \bmod \left(y_{2}, y_{3}, y_{4}\right)^{2}
$$

where $\ell=\ell(P)$ and $i=2($ resp. 3, 4) if $\ell \equiv 2($ resp. 1, 0) $\bmod 3$ [Mor88, (2.16)]. If $\delta_{3}\left(y_{1}, y_{2}\right)$ is square free (resp. has a double factor, is a cube of a linear form), then $(X, P)$ is said to be a simple (resp. double, triple) $\mathrm{cD} / 3$ point.

Extremal curve germs containing a terminal singular point of type $\mathrm{cD} / 3$ are described by the following theorem.

11.5.2. Theorem ([KM92, Th. 6.2-6.3], [MP11, Th. 4.5, 4.8]). Let $f:\left(X, C \simeq \mathbb{P}^{1}\right) \rightarrow(Z, o)$ be an extremal curve germ having a point $P$ of type $\mathrm{cD} / 3$. Then $f$ is a birational contraction, not a $\mathbb{Q}$-conic bundle. General members $H \in\left|\mathscr{O}_{X}\right|$ and $H_{Z}=f(H) \in\left|\mathscr{O}_{Z}\right|$ are normal and have only rational singularities. We have the following possibilities for graphs $\Delta(H, C)$ and $\Delta\left(H_{Z}, o\right)$ and local invariants.

\begin{tabular}{l|l|l|l|} 
No. $|\ell(P)| i_{P}(1) \mid \Delta(H, C)$ & $\Delta\left(H_{Z}, o\right)$ \\
\hline \multicolumn{3}{c}{ Cases of simple cD/3 point $P$} \\
\hline
\end{tabular}




\begin{tabular}{|c|c|c|c|c|c|}
\hline No. & $\ell(P)$ & $i_{P}(1)$ & $\Delta(H, C)$ & $\Delta\left(H_{Z}, o\right)$ & \\
\hline 11.5 .3 & 2 & 1 & 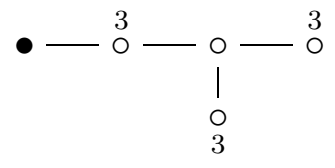 & 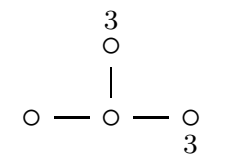 & f \\
\hline 11.5 .4 & 2 & 1 & 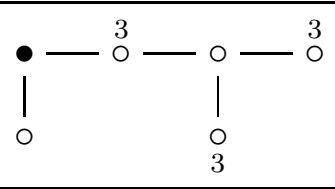 & $\mathrm{A}_{2}$ & $\mathrm{~d}$ \\
\hline \multicolumn{6}{|c|}{ Cases of double $\mathrm{cD} / 3$ point $P$} \\
\hline 11.5 .5 & 2 & 1 & $\begin{array}{l}\dot{1} \\
0 \\
3\end{array}-\circ-\left.\right|_{0} ^{\circ}-0$ & $\begin{array}{l}0 \\
1 \\
0- \\
0 \\
0 \\
3 \\
1 \\
0\end{array}-0$ & $f$ \\
\hline 11.5 .6 & 2 & 1 & $\begin{array}{l}0-0 \\
0 \\
0 \\
3\end{array}-0-\begin{array}{l}0 \\
0 \\
3 \\
1 \\
0\end{array}-0$ & $\mathrm{D}_{4}$ & $\mathrm{~d}$ \\
\hline 11.5 .7 & 3,4 & 2 & $\left.\left.\right|_{0}\right|_{0} ^{\circ}-{ }_{0}^{3}$ & $\left.\right|_{0} ^{0}-0-\frac{0}{3}$ & $\mathrm{f}$ \\
\hline \multicolumn{6}{|c|}{ Case of triple $\mathrm{cD} / 3$ point $P$} \\
\hline 11.5 .8 & 3,4 & 2 & 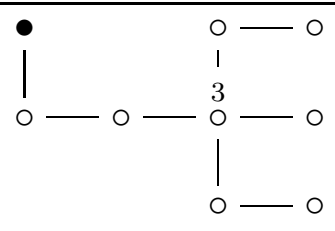 & $\overline{\mathrm{E}_{6}}$ & $\mathrm{~d}$ \\
\hline
\end{tabular}

In the cases 11.5.3, 11.5.5, 11.5.7, and 11.5.8 the variety $X$ is smooth outside $P$ and in the cases [11.5.4 and 11.5.6 X may has at most one type (III) point. The last column indicates if the germ is flipping (f) or divisorial (d).

Note that [KM92, § 6] and [MP11, § 4] provide much more information about these contractions: infinitesimal structure, criterion for an arbitrary germ to be of the corresponding type, and computations of flipped varieties [KM92, A.1]. Flipping contractions can be constructed explicitly by patching certain open subsets:

11.5.9. Example ([KM92, 6.11]). Let $V \supset C$ be a germ of a smooth threefold along $C \simeq \mathbb{P}^{1}$ such that $\mathscr{N}_{C / V} \simeq \mathscr{O}_{C} \oplus \mathscr{O}_{C}$. Pick a point $P \in C$ and let $\left(v_{1}, v_{2}, v_{3}\right)$ be coordinates at $(V, P)$ such that $(C, P)=\left\{v_{1}\right.$-axis $\}$. 
Let $(X, C, P)$ be a $\mathrm{cD} / 3$ point as in (11.5.1) with $\ell=2$. For suitable $\varepsilon_{1}$ and $\varepsilon_{2}$ such that $0<\varepsilon_{1}<\varepsilon_{1} \ll 1,\left(y_{1}^{3}, y_{4}, y_{1} y_{3}\right)$ form coordinates for $U=(X, P) \cap\left\{\varepsilon_{1}<\left|y_{1}^{3}\right|<\varepsilon_{2}\right\}$ by the implicit function theorem. Thus $v_{1}=y_{1}^{3}, v_{2}=y_{4}$, and $v_{3}=y_{1} y_{3}$ patch $(X, P)$ and $V \backslash(V, P) \cap\left\{\left|v_{1}\right|<\varepsilon_{1}\right\}$ along $U$. By [KM92, 6.2.4] the germ $(X, C)$ is a flipping curve germ of type $\mathrm{cD} / 3$ as in 11.5.3 or 11.5.5 (depending on the choice of $\delta_{3}$ in (11.5.1) ).

More examples of flipping contractions are given in [KM92, 6.17 and 6.21]. To show that all the possibilities in Theorem 11.5.2 occur one can also use the deformation arguments 6.6.

11.5.10. Example. Consider the surface contraction $f_{H}: H \rightarrow H_{Z}$ with dual graph 11.5.8 and consider the following triple of germs:

$$
(X, H, P)=\left(\left\{y_{2}^{3}+y_{3}^{3}+y_{3} y_{1}^{4}+y_{4}^{2}\right\},\left\{y_{4}=y_{1} y_{3}\right\}, 0\right) / \boldsymbol{\mu}_{3}(1,1,2,0),
$$

where $H$ is cut out by $y_{4}=y_{1} y_{3}$. Here $(X, P)$ is a triple $\mathrm{cD} / 3$-singularity (see 11.5.1). By [MP11, 4.12] the dual graph of the minimal resolution of $(H, P)$ is the same as that in 11.5.8. By 6.6 one obtains a birational contraction $f: X \rightarrow Z$ extending $f_{H}: H \rightarrow H_{Z}$, which is as in 11.5.8, Examples similar to 11.5.4 and 11.5.6 are given in [MP11, 4.14].

Divisorial contractions of type 11.5.8 were studied also in Tzi10, $5.1(2)]$ by a different method.

\section{CASE (IIA)}

12.1. Setup. Let $(X, C)$ be an extremal curve germ and let $f:(X, C) \rightarrow(Z, o)$ be the corresponding contraction. Assume that $(X, C)$ has a point $P$ of type (IIA). Then by [Mor88, 6.7, 9.4] and [MP08a, 8.6, 9.1, 10.7] $P$ is the only non-Gorenstein point of $X$ and $(X, C)$ has at most one Gorenstein singular point $R$ [Mor88, 6.2], [MP08a, 9.3]. Since $P \in(X, C)$ is locally primitive, the topological index of $(X, C)$ equals 1 . Hence the base $(Z, o)$ is smooth in the $\mathbb{Q}$ conic bundle case, and is a cDV point (or smooth) in the divisorial case (cf. 6.4).

12.2. According to [Mor88, A.3] we can express the (IIA) point as

$$
\begin{aligned}
(X, P) & =\{\alpha=0\} / \boldsymbol{\mu}_{4}(1,1,3,2) \subset \mathbb{C}_{y_{1}, \ldots, y_{4}}^{4} / \boldsymbol{\mu}_{4}(1,1,3,2), \\
C & =\left\{y_{1} \text {-axis }\right\} / \boldsymbol{\mu}_{4},
\end{aligned}
$$

where $\alpha=\alpha\left(y_{1}, \ldots, y_{4}\right)$ is a semi-invariant such that

(12.2.2) $\quad$ wt $\alpha \equiv 2 \bmod 4, \quad \alpha \equiv y_{1}^{\ell(P)} y_{j} \quad \bmod \left(y_{2}, y_{3}, y_{4}\right)^{2}$,

where $j=2($ resp. 3, 4) if $\ell(P) \equiv 1($ resp. 3,0) $\bmod 4($ see (3.3.1) $)$ and $\left(\mathcal{J}_{C}^{\sharp}\right)^{(2)}=\left(y_{j}\right)+\left(\mathcal{J}_{C}^{\sharp}\right)^{2}$. Moreover, $y_{2}^{2}, y_{3}^{2} \in \alpha$ (because $(X, P)$ is a terminal point of type $\mathrm{cAx} / 4)$. Note that $\ell(P) \not \equiv 2 \bmod 4$ because of the lack of a variable with wt $\equiv 0 \bmod 4$. 
12.3. Theorem ([KM92, 7.2-7.4], MP16, MP17]). Let $f:(X, C \simeq$ $\left.\mathbb{P}^{1}\right) \rightarrow(Z, o)$ be an extremal curve germ having a point $P$ of type (IIA). We have the following possibilities for graphs $\Delta(H, C)$ and $\Delta\left(H_{Z}, o\right)$ and local invariants.

\begin{tabular}{|c|c|c|c|c|c|}
\hline No. & $i_{P}(1$ & $(P)$ & $\Delta(H, C)$ & $\Delta\left(H_{Z}, o\right)$ & \\
\hline \multicolumn{6}{|c|}{ Cases: $H$ is normal } \\
\hline 12.3.1 & 1 & 1 & 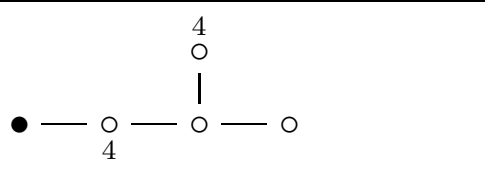 & 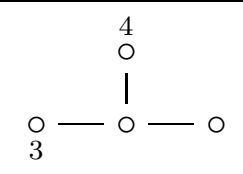 & $\mathrm{f}$ \\
\hline 12.3 .2 & 1 & 1 & 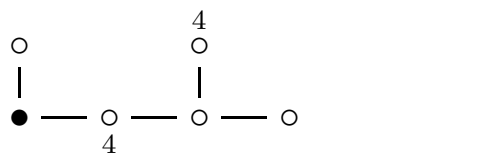 & $\begin{array}{r}4 \\
0 \\
1 \\
-0\end{array}$ & $\mathrm{f}$ \\
\hline 12.3 .3 & 2 & 3,4 & 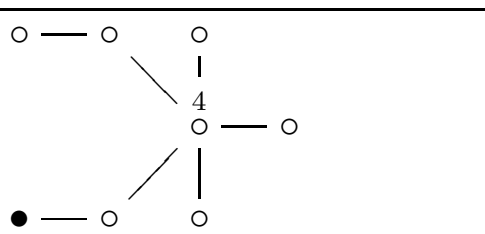 & 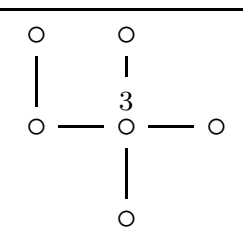 & $\mathrm{f}$ \\
\hline 12.3 .4 & 1 & 1 & 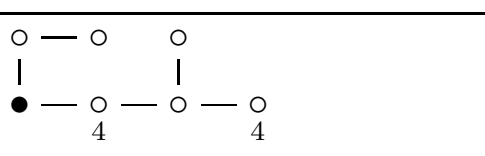 & $\overline{A_{1}}$ & $\mathrm{~d}$ \\
\hline 12.3 .5 & 1 & 1 & $\begin{array}{l}0 \\
\text { l } \\
\bullet-0\end{array}$ & $\mathrm{~A}_{1}$ & d \\
\hline 12.3 .6 & 2 & 3,5 & 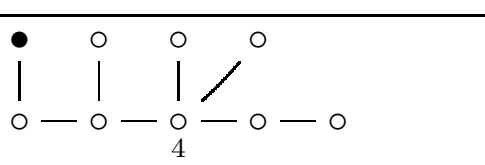 & $\overline{D_{5}}$ & $\mathrm{~d}$ \\
\hline 12.3 .7 & 2 & 4,5 & 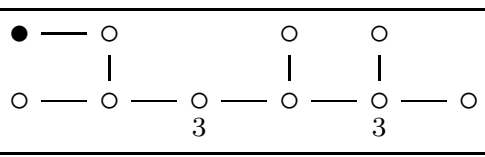 & & $\mathrm{c}$ \\
\hline \multicolumn{6}{|c|}{ Cases: $H$ is not normal } \\
\hline 12.3 .8 I & & & $\bullet-\begin{array}{l}\circ \\
\mid \\
0 \\
3 \\
1 \\
0\end{array}$ & $\mathrm{D}_{5}$ & $\mathrm{~d}$ \\
\hline 12.3 .9 & & & $\circ-o_{0}^{3}-\circ$ & & $\mathrm{C}$ \\
\hline
\end{tabular}

\footnotetext{
${ }^{1}$ This case was erroneously omitted in Tzi05a, Th. 3.6 and Cor. 3.8].
} 
The variety $X$ can have (at most one) extra type (III) singular point in all cases except for 12.3.1, 12.3.3, 12.3.6, and 12.3.7 where the singular point is unique.

Examples of flipping contractions can be constructed similar to 11.5.9.

12.4. Example ([KM92, 7.6.4]). Let $V \supset C$ be a germ of a smooth threefold along $C \simeq \mathbb{P}^{1}$ such that $\mathscr{N}_{C / V} \simeq \mathscr{O}_{C} \oplus \mathscr{O}_{C}$. Pick a point $P \in C$ and let $\left(v_{1}, v_{2}, v_{3}\right)$ be coordinates at $(V, P)$ such that $(C, P)=\left\{v_{1}\right.$-axis $\}$. Let $(X, C, P)$ be a (IIA)-point as in (12.2.1)-(12.2.2) with $\alpha \equiv y_{1} y_{2}$ $\bmod \left(y_{2}, y_{3}, y_{4}\right)^{2}$. For suitable $\varepsilon_{1}$ and $\varepsilon_{2}$ such that $0<\varepsilon_{1}<\varepsilon_{1} \ll 1$, $\left(y_{1}^{4}, y_{1}^{2} y_{4}, y_{1} y_{3}\right)$ form coordinates for $U=(X, P) \cap\left\{\varepsilon_{1}<\left|y_{1}^{4}\right|<\varepsilon_{2}\right\}$ by the implicit function theorem. Thus $v_{1}=y_{1}^{4}, v_{2}=y_{1}^{2} y_{4}$, and $v_{3}=y_{1} y_{3}$ patch $(X, P)$ and $V \backslash(V, P) \cap\left\{\left|v_{1}\right|<\varepsilon_{1}\right\}$ along $U$. By [KM92, 7.2.4] the germ $(X, C)$ is a flipping curve germ of type (IIA) as in 12.3.1. See KM92, 7.9.4, 7.12.5] for more examples of flipping contractions.

The existence in the above theorem in the case where $H$ is normal can be established by using arguments of 6.6. In the case 12.3.6 we have also explicit example:

12.5. Example $([\mathrm{MP} 16,6.6])$. Let $Z \subset \mathbb{C}_{z_{1}, \ldots, z_{5}}^{5}$ be defined by two equations:

$$
\begin{aligned}
& 0=z_{2}^{2}+z_{3}+z_{4} z_{5}^{k}+z_{1}^{3}, \quad k \geq 1, \\
& 0=z_{1}^{2} z_{2}^{2}+z_{4}^{2}-z_{3} z_{5}+z_{1}^{3} z_{2}+c z_{1}^{2} z_{4} .
\end{aligned}
$$

By eliminating $z_{3}$ using the first equation, one sees easily that $(Z, 0)$ is a threefold singularity of type $\mathrm{cD}_{5}$. Let $B \subset Z$ be the $z_{5}$-axis, and let $f: X \rightarrow Z$ be the weighted blowup of $B$ with weight $(1,1,4,2,0)$. By an easy computation one sees that $C:=f^{-1}(0)_{\text {red }} \simeq \mathbb{P}^{1}$ and $X$ is covered by two charts: $z_{1}$-chart and $z_{3}$-chart. The origin of the $z_{3}$-chart is a type (IIA) point $P$ with $\ell(P)=3$ :

$$
\left\{y_{1}^{3} y_{3}+y_{2}^{2}+y_{3}^{2}+y_{4}\left(y_{1}^{2} y_{2}^{2}+y_{4}^{2}+y_{1}^{3} y_{2}+c y_{1}^{2} y_{4}\right)^{k}=0\right\} / \boldsymbol{\mu}_{4}(1,1,3,2),
$$

where $(C, P)$ is the $y_{1}$-axis. Moreover, $X$ is smooth outside $P$. Thus $X \rightarrow Z$ is a divisorial contraction of type 12.3.6. See also [MP16, 8.3.3] for an example with $\ell(P)=5$.

The case 12.3.6 was also studied by N. Tziolas [Tzi05a, Th. 3.6].

The existence of 12.3 .8 can be shown similar to Example 12.5.

12.6. Example $([\mathrm{MP17}, 3.6])$. Let $Z \subset \mathbb{C}_{z_{1}, \ldots, z_{5}}^{5}$ be defined by

$$
\begin{aligned}
& 0=z_{2}^{2}+z_{3}+z_{4} z_{5}^{k}-z_{1}^{3}, \quad k \geq 1, \\
& 0=z_{1}^{2} z_{2}^{2}+z_{4}^{2}-z_{3} z_{5} .
\end{aligned}
$$

Then $(Z, 0)$ is a threefold singularity of type $\mathrm{cD}_{5}$. Let $B \subset Z$ be the $z_{5}$-axis and let $f: X \rightarrow Z$ be the weighted (1,1,4,2,0)-blowup. The 
origin of the $z_{3}$-chart is a type (IIA) point $P$ with $\ell(P)=3$ :

$$
\left\{-y_{1}^{3} y_{3}+y_{2}^{2}+y_{3}^{2}+y_{4}\left(y_{1}^{2} y_{2}^{2}+y_{4}^{2}\right)^{k}=0\right\} / \boldsymbol{\mu}_{4}(1,1,3,2),
$$

where $(C, P)$ is the $y_{1}$-axis. In the $z_{1}$-chart we have a type (III) point. See also [MP17, 3.7] for an example of a divisorial germ as in 12.3.8 whose singular locus consists of a single (IIA) point $P$ with $\ell(P)=7$.

12.7. Example ([MP17, 4.8]). Let $X$ be the the hypersurface of weighted degree 10 in the weighted projective space $\mathbb{P}(1,1,3,2,4)_{x_{1}, \ldots, x_{4}, w}$ given by the equation

$$
w \phi_{6}-x_{1}^{6} \phi_{4}=0, \quad \text { where } \quad \begin{aligned}
& \phi_{6}:=x_{1}^{4} x_{4}+x_{3}^{2}+x_{2}^{2} w+\delta x_{4}^{3}, \\
& \phi_{4}:=x_{4}^{2}+\nu x_{2} x_{3}+\eta x_{1}^{2} x_{4}+\mu x_{1}^{3} x_{2}
\end{aligned}
$$

(for simplicity we assume that the coefficients $\delta, \nu, \eta$ are general). Regard $X$ as a small analytic neighborhood of $C$. In the affine chart $U_{w}:=\{w \neq 0\} \simeq \mathbb{C}^{4} / \boldsymbol{\mu}_{4}(1,1,3,2)$ the variety $X$ is given by

$$
\phi_{6}\left(y_{1}, y_{2}, y_{3}, y_{4}, 1\right)-y_{1}^{6} \phi_{4}\left(y_{1}, y_{2}, y_{3}, y_{4}, 1\right)=0
$$

and $C$ is the $y_{1}$-axis. Clearly, it has the form (12.2.2). So, the origin $P \in(X, C)$ is a type (IIA) point with $\ell(P)=4$.

In the affine chart $U_{1}:=\left\{x_{1} \neq 0\right\} \simeq \mathbb{C}^{4}$ the variety $X$ is defined by

$$
w \phi_{6}\left(1, z_{2}, z_{3}, z_{4}, w\right)-\phi_{4}\left(1, z_{2}, z_{3}, z_{4}, w\right)=0 .
$$

If $\mu \neq 0$, then $X$ is smooth outside $P$, i.e. $(X, C)$ is as in the case 12.3.7. If $\mu=0$, then $(X, C)$ has a type (III) point at $(0,0,0, \eta)$.

We claim that $(X, C)$ admits a structure of a $\mathbb{Q}$-conic bundle germ as in 12.3.9 (resp. 12.3.7) if $\mu=0$ (resp. $\mu \neq 0$ ).

Proof. Consider the surface $H=\left\{\phi_{6}=\phi_{4}=0\right\} \subset X$. Let $\psi: H^{\mathrm{n}} \rightarrow H$ be the normalization (we put $H^{\mathrm{n}}=H$ if $H$ is normal) and let $C^{\mathrm{n}}:=\psi^{-1}(C)$. One can explicitly check that $H$ is normal and smooth outside $P$ if $\mu \neq 0$ and $H$ is singular along $C$, the curve $C^{\text {n }}$ is irreducible and rational, and $\psi_{C}:=C^{\mathrm{n}} \rightarrow C$ is a double cover if $\mu=0$. Moreover, the singularities of $H^{\mathrm{n}}$ are rational. Note that $H$ is a fiber of the fibration $\pi: X \rightarrow D$ over a small disk around the origin given by the rational function $\phi_{4} / w=\phi_{6} / x_{1}^{6}$ which is regular in a neighborhood of $C$. Analyzing the minimal resolution one can show that there exists a rational curve fibration $f_{H}: H \rightarrow B$, where $B \subset \mathbb{C}$ is a small disk around the origin, such that $C=f_{H}^{-1}(0)_{\text {red }}$. Now the existence of a contraction is a consequence of the following.

12.7.1. Claim.

(i) $H^{1}\left(\hat{X}, \mathscr{O}_{\hat{X}}\right)=0$, where $\hat{X}$ denotes the completion of $X$ along $C$.

(ii) The contraction $f_{H}: H \rightarrow B$ extends to a contraction $\hat{f}: \hat{X} \rightarrow \hat{Z}$. 
(iii) There exists a contraction $f: X \rightarrow Z$ that approximates $\hat{f}: \hat{X} \rightarrow \hat{Z}$.

Proof. For (i) we refer to [MP17, 4.8.4].

(ii) Since $H^{1}\left(\mathscr{O}_{\hat{X}}\right)=0$, from the exact sequence

$$
0 \longrightarrow \mathscr{O}_{X} \longrightarrow \mathscr{O}_{X}(H) \longrightarrow \mathscr{O}_{H}(H) \longrightarrow 0
$$

we see that the map $H^{0}\left(\mathscr{O}_{\hat{X}}(\hat{H})\right) \rightarrow H^{0}\left(\mathscr{O}_{\hat{H}}(\hat{H})\right)$ is surjective. Hence there exists a divisor $\hat{H}_{1} \in\left|\mathscr{O}_{\hat{X}}\right|$ such that $\left.\hat{H}_{1}\right|_{\hat{H}}=\hat{\mathscr{C}}$. Then the divisors $\hat{H}$ and $\hat{H}_{1}$ define a contraction $\hat{f}: \hat{X} \rightarrow \hat{Z}$.

(iii) Let $F$ be the scheme fiber of $f_{H}: H \rightarrow B$ over the origin. The above arguments shows that the deformations of $F$ are unobstructed. Therefore the corresponding component of the Douady space is smooth and two-dimensional. This allow us to produce a contraction on $X$.

12.8. Example ([MP17, 4.9]). Similarly to Example [12.7, let $X \subset$ $\mathbb{P}(1,1,3,2,4)$ be a small analytic neighborhood of $C=\left\{\left(x_{1}, w\right)\right.$-line $\}$ given by the equation $x_{1}^{6} \phi_{4}-w \phi_{6}=0$, where

$$
\begin{aligned}
& \phi_{6}:=x_{3}^{2}+x_{2}^{2} w+\delta x_{4}^{3}+c x_{1}^{2} x_{4}^{2}, \\
& \phi_{4}:=x_{4}^{2}+\nu x_{2} x_{3}+\eta x_{1}^{2} x_{4} .
\end{aligned}
$$

It is easy to check that $P:=(0: 0: 0: 0: 1)$ is the only singular point of $X$ on $C$ and it is a type (IIA) point with $\ell(P)=8$. The rational function $\phi_{4} / w=\phi_{6} / x_{1}^{6}$ near $C$ defines a fibration whose central fiber $H$ is given by $\phi_{4}=\phi_{6}=0$ such that $\Delta(H, C)$ is of type 12.3.9. The existence of a contraction $f: X \rightarrow Z$ can be shown similar to Claim 12.7.1.

12.9. Example ([MP17, 4.9.1]). In a similar way we can construct an example of a $\mathbb{Q}$-conic bundle with $\ell(P)=5$ and normal $H$ as in 12.3.7. Consider $X \subset \mathbb{P}(1,1,3,2,4)$ given by $w \phi_{6}-x_{1}^{6} \phi_{4}=0$, where

$$
\phi_{6}:=x_{1}^{5} x_{2}+x_{2}^{2} w+x_{3}^{2}+\delta x_{4}^{3}+c x_{1}^{2} x_{4}^{2}
$$

and $\phi_{4}$ is as in 12.7. In the affine chart $U_{w} \simeq \mathbb{C}^{4} / \boldsymbol{\mu}_{4}(1,1,3,2)$ the origin $P \in(X, C)$ is a type (IIA) point with $\ell(P)=5$. It is easy to see that $X$ is smooth outside $P$. The rational function $\phi_{4} / w=\phi_{6} / x_{1}^{6}$ defines a fibration on $X$ near $C$ with central fiber $H=\left\{\phi_{4}=\phi_{6}=0\right\}$.

\section{Appendix A. A REMARK on Divisorial CONTRACTIONS}

A.1. Proposition. Let $\left(X, C \simeq \mathbb{P}^{1}\right)$ be a divisorial curve germ with one non-Gorenstein point which is not of type $\mathrm{cA} / \mathrm{m}$ with $m>2$ and let $f:(X, C) \rightarrow(Z, o)$ be corresponding contraction. Let $E \subset X$ be the exceptional divisor and let $B:=f(E)$ be the blowup curve. Then the multiplicity mult $_{o}(B)$ is given by the following table. 


\begin{tabular}{lllll}
$(X, C)$ & $\Delta(H, C)$ & mult $_{o}(B)$ & $H_{Z}$ & $D_{Z}$ \\
\hline (IIA) & $12.3 .4,12.3 .5$ & 3 & $\mathrm{~A}_{1}$ & $\mathrm{D}_{2 \mathrm{n}+1}$ \\
$(\mathrm{IIA})$ & $12.3 .6,12.3 .8$ & 1 & $\mathrm{D}_{5}$ & $\mathrm{D}_{2 \mathrm{n}+1}$ \\
$(\mathrm{IIB})$ & $10.2 .1,10.2 .3$ & 2 & $\mathrm{~A}_{2}, \mathrm{D}_{4}$ & $\mathrm{E}_{6}$ \\
$(\mathrm{IIB})$ & 10.2 .2 & 5 & $\mathrm{~A}_{0}$ & $\mathrm{E}_{6}$ \\
$\mathrm{cD} / 3$ & $11.5 .4,11.5 .6$ & 2 & $\mathrm{~A}_{2}, \mathrm{D}_{4}$ & $\mathrm{E}_{6}$ \\
$\mathrm{cD} / 3$ & 11.5 .8 & 1 & $\mathrm{E}_{6}$ & $\mathrm{E}_{6}$ \\
$\mathrm{cA} / 2$ & 8.2 .2 & $n$ & $\mathrm{~A}_{1}$ & $\mathrm{~A}$ \\
$\mathrm{cA} / 2$ & 8.2 .3 & 3 & $\mathrm{~A}_{0}$ & $\mathrm{~A}$ \\
$\mathrm{cA} / 2$ & 8.2 .4 & 1 & $\mathrm{~A}_{2}$ & $\mathrm{~A}$ \\
$\mathrm{cA} / 2$ & 8.2 .5 & 4 & $\mathrm{~A}_{0}$ & $\mathrm{~A}$ \\
$\mathrm{cAx} / 2, \mathrm{cD} / 2$ & 8.2 .118 .2 .12 & 1 & $\mathrm{D}$ & $\mathrm{D}$ \\
$\mathrm{cE} / 2$ & 8.2 .118 .2 .13 & 1 & $\mathrm{D}, \mathrm{E}_{6}$ & $\mathrm{E}_{7}$
\end{tabular}

where $H_{Z}$ is a general hyperplane section of $(Z, o)$ and $D_{Z}$ is a general hyperplane section of $(Z, o)$ passing through $B$. In the $\mathrm{cA} / 2$-case the meaning of $n$ is the same as in 8.2 .2 .

The cases with $\operatorname{mult}_{o}(B)=1$, i.e. those with smooth $B$, were studied in details by N. Tziolas [Tzi03], [Tzi05b], [Tzi05a], TTzi10].

Proof. Recall that $Z$ is $\mathbb{Q}$-Gorenstein and $E$ is $\mathbb{Q}$-Cartier divisor (Theorem 6.4). By classification in all our cases $Z$ is in fact Gorenstein (that is, $H_{Z}$ has at worst Du Val singularity). Hence, $E \in\left|K_{X}\right|$. Let $H:=f^{*}\left(H_{Z}\right)$. Let $D \in\left|-K_{X}\right|$ be a general member. We have $-K_{X} \cdot C=1 / m$, where $m$ is the index of the non-Gorenstein point (see 4.7.4). For simplicity assume that $H$ is normal. The case 12.3.8 can be treated in a similar way.

A.2. Lemma ([Tzi05b, Lemma 5.1]). If in the above notation $H$ is normal, then

$$
\operatorname{mult}_{o}(B)=-\frac{\left(K_{C} \cdot C\right)^{2}}{\left(C^{2}\right)_{H}}=-\frac{1}{m^{2}\left(C^{2}\right)_{H}} .
$$

Now let $\psi: \hat{H} \rightarrow H$ be the minimal resolution. Write $\psi^{*} C=\hat{C}+\Theta$, where $\operatorname{Supp}(\Theta) \subset \operatorname{Exc}(\psi)$ and $\Theta=\sum \theta_{i} \Theta_{i}$. Since $\hat{C}^{2}=-1$, we have

$$
C^{2}=-1+\hat{C} \cdot \Theta=-1+\sum^{\prime} \theta_{i},
$$

where $\sum^{\prime}$ runs through all the components $\Theta_{i}$ meeting $\hat{C}$. The coefficients $\theta_{i}$ are computed from the standard system of linear equations:

$$
0=-\Theta_{j} \cdot \psi^{*} C=\Theta_{j} \cdot \hat{C}+\sum_{i} \theta_{i} \Theta_{j} \cdot \Theta_{i} .
$$

Now mult $_{o}(B)$ can be computed by using (A.2.2). Consider for example the cases 12.3.4, 12.3.5 and 12.3.6 of Theorem 12.3 (other cases are similar). In the graphs below we attach the coefficients $\theta_{i}$ of $\Theta=\psi^{*} C-$ 
$\hat{C}$ to the corresponding vertices and indicate the value of $C^{2}$. This immediately gives us the values of $\operatorname{mult}_{o}(B)$ as desired.

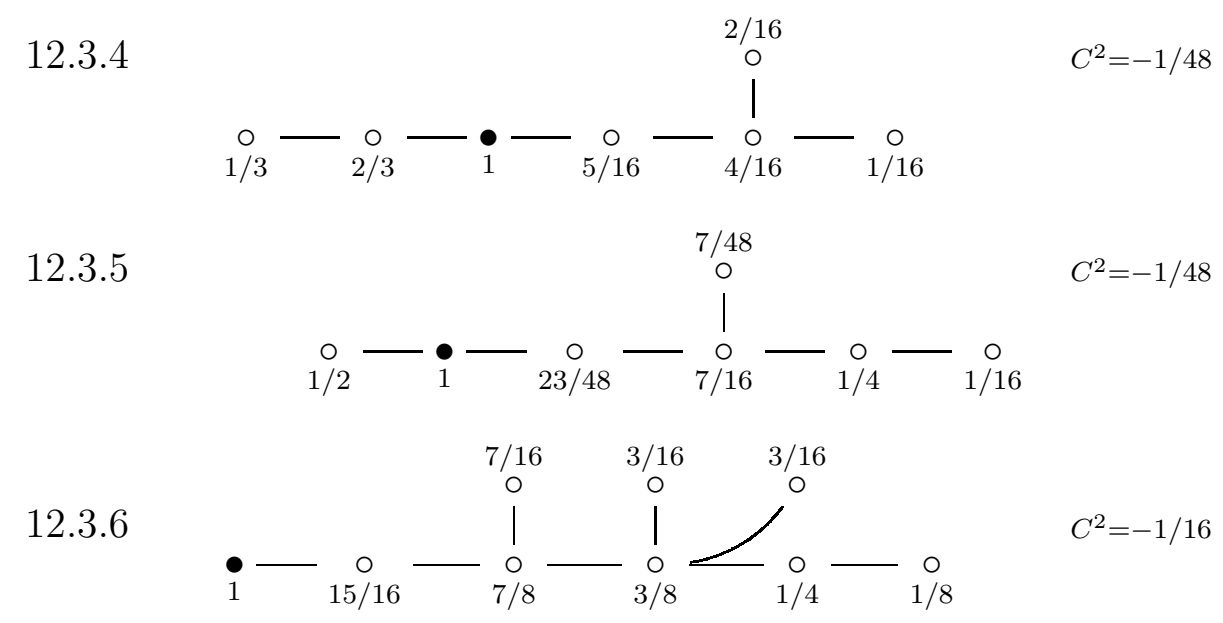

\section{REFERENCES}

[CKM88] Herbert Clemens, János Kollár, and Shigefumi Mori. Higher-dimensional complex geometry. Astérisque, (166):144 pp. (1989), 1988.

[Cut88] Steven Cutkosky. Elementary contractions of Gorenstein threefolds. Math. Ann., 280(3):521-525, 1988.

[Duc16] Tom Ducat. Divisorial extractions from singular curves in smooth 3-folds. Internat. J. Math., 27(1):1650005, 23, 2016.

[Feu71] R. D. Feuer. Torsion-free subgroups of triangle groups. Proc. Amer. Math. Soc., 30:235-240, 1971.

[HTU17] Paul Hacking, Jenia Tevelev, and Giancarlo Urzúa. Flipping surfaces. J. Algebraic Geom., 26(2):279-345, 2017.

[Ish14] Shihoko Ishii. Introduction to singularities. Springer, Tokyo, 2014.

[Isk96] V. A. Iskovskikh. On a rationality criterion for conic bundles. Sbornik: Math., 187(7):1021-1038, 1996.

[Kaw88] Yujiro Kawamata. Crepant blowing-up of 3-dimensional canonical singularities and its application to degenerations of surfaces. Ann. of Math. (2), 127(1):93-163, 1988.

[Kaw92] Yujiro Kawamata. The minimal discrepancy coefficients of terminal singularities in dimension three. Appendix to V.V. Shokurov's paper "3-fold log flips". Russ. Acad. Sci., Izv., Math., 40(1):95-202, 1992.

[Kaw96] Yujiro Kawamata. Divisorial contractions to 3-dimensional terminal quotient singularities. In Higher-dimensional complex varieties (Trento, 1994), pages 241-246. de Gruyter, Berlin, 1996.

[Kaw97] Yujiro Kawamata. On Fujita's freeness conjecture for 3-folds and 4-folds. Math. Ann., 308(3):491-505, 1997.

[Kaw07] Masayuki Kawakita. Inversion of adjunction on log canonicity. Invent. Math., 167:129-133, 2007.

[KM92] János Kollár and Shigefumi Mori. Classification of three-dimensional flips. J. Amer. Math. Soc., 5(3):533-703, 1992. 
[KM98] János Kollár and Shigefumi Mori. Birational geometry of algebraic varieties, volume 134 of Cambridge Tracts in Mathematics. Cambridge University Press, Cambridge, 1998. With the collaboration of C. H. Clemens and A. Corti, Translated from the 1998 Japanese original.

[Kol86] János Kollár. Higher direct images of dualizing sheaves. I. Ann. of Math. (2), 123(1):11-42, 1986.

[Kol92] János Kollár, editor. Flips and abundance for algebraic threefolds. Société Mathématique de France, Paris, 1992. Papers from the Second Summer Seminar on Algebraic Geometry held at the University of Utah, Salt Lake City, Utah, August 1991, Astérisque No. 211 (1992).

[Kol99] János Kollár. Real algebraic threefolds. III. Conic bundles. J. Math. Sci. (New York), 94(1):996-1020, 1999. Algebraic geometry, 9.

[KSB88] J. Kollár and N. I. Shepherd-Barron. Threefolds and deformations of surface singularities. Invent. Math., 91(2):299-338, 1988.

[LW86] Eduard Looijenga and Jonathan Wahl. Quadratic functions and smoothing surface singularities. Topology, 25(3):261-291, 1986.

[Mor75] Shigefumi Mori. On a generalization of complete intersections. J. Math. Kyoto Univ., 15(3):619-646, 1975.

[Mor85] Shigefumi Mori. On 3-dimensional terminal singularities. Nagoya Math. J., 98:43-66, 1985.

[Mor88] Shigefumi Mori. Flip theorem and the existence of minimal models for 3-folds. J. Amer. Math. Soc., 1(1):117-253, 1988.

[Mor02] Shigefumi Mori. On semistable extremal neighborhoods. In Higher dimensional birational geometry (Kyoto, 1997), volume 35 of Adv. Stud. Pure Math., pages 157-184. Math. Soc. Japan, Tokyo, 2002.

[MP08a] Shigefumi Mori and Yuri Prokhorov. On Q-conic bundles. Publ. Res. Inst. Math. Sci., 44(2):315-369, 2008.

[MP08b] Shigefumi Mori and Yuri Prokhorov. On Q-conic bundles. II. Publ. Res. Inst. Math. Sci., 44(3):955-971, 2008.

[MP09] S. Mori and Yu. Prokhorov. On Q-conic bundles, III. Publ. Res. Inst. Math. Sci., 45(3):787-810, 2009.

[MP11] S. Mori and Yu. Prokhorov. Threefold extremal contractions of type IA. Kyoto J. Math., 51(2):393-438, 2011.

[MP14] S. Mori and Yu. Prokhorov. Threefold extremal contractions of types (IC) and (IIB). Proc. Edinburgh Math. Soc., 57(1):231-252, 2014.

[MP16] S. Mori and Yu. Prokhorov. Threefold extremal contractions of type (IIA), I. Izv. Math., 80(5):884-909, 2016.

[MP17] Shigefumi Mori and Yuri Prokhorov. Threefold extremal contractions of type (IIA), II. ArXiv e-print, 1707.05047, 2017. to appear in Festschrift in honour of Nigel Hitchin, (2018), Oxford University Press.

[PR16] Yuri Prokhorov and Miles Reid. On Q-Fano threefolds of Fano index 2. In Minimal Models and Extremal Rays (Kyoto 2011), volume 70 of Adv. Stud. Pure Math., pages 397-420. Mathematical Society of Japan, Kinokuniya, Tokyo, 2016.

[Pro97] Yu. Prokhorov. On the existence of complements of the canonical divisor for Mori conic bundles. Russian Acad. Sci. Sb. Math., 188(11):1665-1685, 1997.

[Pro04] Yu. Prokhorov. On semistable Mori contractions. Izv. Math., 68(2):147158, 2004.

[Pro18] Yuri Prokhorov. The rationality problem for conic bundles. Russian Math. Surveys, 73(3), 2018. 
[Rei83] Miles Reid. Minimal models of canonical 3-folds. In Algebraic varieties and analytic varieties (Tokyo, 1981), volume 1 of Adv. Stud. Pure Math., pages 131-180. North-Holland, Amsterdam, 1983.

[Rei87] Miles Reid. Young person's guide to canonical singularities. In Algebraic geometry, Bowdoin, 1985 (Brunswick, Maine, 1985), volume 46 of Proc. Sympos. Pure Math., pages 345-414. Amer. Math. Soc., Providence, RI, 1987.

[SB83] N. I. Shepherd-Barron. Degenerations with numerically effective canonical divisor. In The birational geometry of degenerations (Cambridge, Mass., 1981), volume 29 of Progr. Math., pages 33-84. Birkhäuser Boston, Boston, MA, 1983.

[Sho93] V. V. Shokurov. 3-fold log flips. Russ. Acad. Sci., Izv., Math., 40(1):95202, 1993.

[Tzi03] Nikolaos Tziolas. Terminal 3-fold divisorial contractions of a surface to a curve. I. Compos. Math., 139(3):239-261, 2003.

[Tzi05a] Nikolaos Tziolas. Families of $D$-minimal models and applications to 3-fold divisorial contractions. Proc. Lond. Math. Soc., III. Ser., 90(2):345-370, 2005.

[Tzi05b] Nikolaos Tziolas. Three dimensional divisorial extremal neighborhoods. Math. Ann., 333(2):315-354, 2005.

[Tzi09] Nikolaos Tziolas. Q-Gorenstein deformations of nonnormal surfaces. Amer. J. Math., 131(1):171-193, 2009.

[Tzi10] N. Tziolas. Three-fold divisorial extremal neighborhoods over $c E_{7}$ and $c E_{6}$ compound DuVal singularities. Internat. J. Math., 21(1):1-23, 2010.

Shigefumi Mori: Kyoto University Institute for Advanced Study, KYOTO UNIVERSITY, KYOTO, JAPAN

Research Institute for Mathematical Sciences, Kyoto University, KYOTO, JAPAN

E-mail address: mori@kurims.kyoto-u.ac.jp

Yuri Prokhorov: Steklov Mathematical Institute of Russian Academy of Sciences, Moscow, Russia

Department of Algebra, Moscow State Lomonosov University

National Research University Higher School of Economics

E-mail address: prokhoro@mi.ras.ru 